\title{
A Numerical Scheme for the Quantile Hedging Problem*
}

\author{
Cyril Bénézet ${ }^{\dagger}$, Jean-François Chassagneux ${ }^{\ddagger}$, and Christoph Reisinger ${ }^{\S}$
}

\begin{abstract}
We consider numerical approximations to the quantile hedging price of a European claim in a nonlinear market with Markovian dynamics. We study an equivalent stochastic target problem with the conditional probability of success as a new state variable, in addition to the asset value process. We propose numerical approximations based on piecewise constant policy time stepping coupled with novel finite difference schemes. We prove convergence in the monotone case combining backward stochastic differential equation arguments with the Barles and Jakobsen and Barles and Souganidis approaches for nonlinear PDEs. The difficulties compared to the classical setting consist in the construction of monotone schemes under degeneracy due to the perfectly correlated joint process, the unboundedness of the control variable, and the effect of the boundaries in the probability variable on the analysis. We extend the method to a class of nonmonotone schemes using higher order interpolation and prove convergence for linear drivers. In a numerical section, we illustrate the performance of our schemes by considering an example in a financial market with imperfections, and show that a standard nonmonotone scheme produces financially counterintuitive solutions.
\end{abstract}

Key words. quantile hedging, BSDEs, monotone approximation schemes

AMS subject classifications. 65M06, 65M12, 93E20, 91G60, 60H30

DOI. $10.1137 / 19 \mathrm{M} 1267477$

1. Introduction. In this work, we study numerical approximations to the quantile hedging price of a European contingent claim with maturity $T>0$ in a complete market with possible imperfections. The quantile hedging problem is a specific case of a broader class of approximate hedging problems. It consists in finding the minimal initial endowment of a portfolio that will allow hedging the claim with a given probability of success $p$, the case $p=1$ corresponding to the classical problem of (super)replication. This approach has been popularized by Föllmer and Leukert in [23] who provided a closed form solution in a special setting. The first PDE characterization was introduced by [10] in a possibly incomplete market setting with portfolio constraints. Various extensions have been considered since this work: to jump dynamics [33]; to the Bermudan case [8] and American case [19]; to a non-Markovian setting [9, 18]; and to

\footnotetext{
*Received by the editors June 11, 2019; accepted for publication (in revised form) June 26, 2020; published electronically February 1, 2021

https://doi.org/10.1137/19M1267477

Funding: This work was partially funded within the scope of the research project "Advanced techniques for non-linear pricing and risk management of derivatives" under the aegis of the Europlace Institute of Finance, with the support of AXA Research Fund.

${ }^{\dagger}$ Centre de Mathématiques Appliquées (CMAP), École Polytechnique and CNRS, Université Paris-Saclay, Route de Saclay, 91128 Palaiseau Cedex, France (cyril.benezet@polytechnique.edu).

¥UFR de Mathématiques and LPSM, Université de Paris, Bâtiment Sophie Germain, 8 place Aurélie Nemours, 75013 Paris, France (chassagneux@lpsm.paris).

${ }^{\S}$ Mathematical Institute, University of Oxford, Woodstock Road, Oxford, OX2 6GG, United Kingdom (christoph.reisinger@maths.ox.ac.uk).
} 
a finite number of quantile constraints [11]. Except for [8, 11], all the aforementioned works are theoretical in nature. The lack of established numerical methods for these problems is a clear motivation for our study.

The main objective of this paper is to design a numerical procedure to approximate the quantile hedging pricing function $v:[0, T] \times \mathbb{R}^{d} \times[0,1] \rightarrow \mathbb{R}$ with numerical schemes for the associated nonlinear PDE first derived in [10]. We consider a complete market with nonlinear wealth dynamics, modeling market frictions. A typical example, investigated numerically in section 3 , is the case with constant borrowing and lending rates, $R$ and $r$, respectively, with $R \geq r$ (see [21, Example 1.1]). We refer to subsection 2.1 where we recall the definitions and main properties of $v$ and the PDE.

In the linear market setting, using a dual approach, [8] combines the solution of a linear PDE with Fenchel-Legendre transforms to tackle the problem of Bermudan quantile hedging. This approach cannot be directly adapted here, because the dual approach in the nonlinear setting would require a convexity assumption on the driver of the associated backward stochastic differential equation (BSDE) (while here we only require Lipschitz continuity) and results in fully nonlinear PDEs.

To the best of our knowledge, this is the first numerical method for the quantile hedging problem in this nonlinear market specification. The design and analysis of numerical schemes for the nonlinear case require several extensions to the standard framework for nonlinear PDEs, mainly due to the unboundedness of the controls and the presence of the boundaries in the probability variable. Moreover, we construct a numerical scheme which is more accurate and easier to implement than standard schemes. We use the following strategy:

1. Bound and discretize the set where the controls take their values.

2. Consider an associated piecewise constant policy time stepping (PCPT) scheme for the control processes.

3. Use a monotone finite difference scheme to approximate in time and space the PCPT solution resulting from 1 and 2 .

The approximation of controlled diffusion processes by ones where policies are piecewise constant in time was first analysed by [30]; in [31], this procedure is used in conjunction with Markov chain approximations to diffusion processes to construct fully discrete approximation schemes to the associated Bellman equations and to derive their convergence order. An improvement to the order of convergence from [30] was shown recently in [29] using a refinement of Krylov's original, probabilistic techniques. Using purely viscosity solution arguments for PDEs, error bounds for such approximations are derived in [3], which are weaker than those in [30] for the control approximation scheme, but improve the bounds in [31] for the fully discrete scheme. In [38], using a switching system approximation introduced in [3], convergence is proved for a generalized scheme where linear PDEs are solved piecewise in time on different meshes, and the control optimization is carried out at the end of time intervals using possibly nonmonotone, higher order interpolations. An extension of the analysis in [38] to jump processes and nonlinear expectations is given in [20]. We note that [30, 31, 3] and related works study the problem posed on the whole space, while [38] merely assumes consistency of the scheme at the boundary and in that case does not analyze attainment of the boundary conditions. The only works we are aware of to address strong boundary conditions are [17, 35], but under the assumption of a so-called "barrier function," a smooth strict sub- or super-solution 
satisfying the boundary data. It seems generally difficult to ascertain the existence of such a barrier function, even in the nondegenerate case.

Our first contribution is thus to prove that the approximations built in step 1 and 2 above are convergent for the quantile hedging problem, which has substantial new difficulties compared to the settings considered in the aforementioned works. For this we heavily rely on the comparison theorem for the formulation in (2.3) and we take advantage of the monotonicity property of the approximating sequences. The main new difficulties come from the nonlinear form of the PDE including unbounded controls, and in particular the boundaries in the probability variable. To deal with the latter especially, we rely on some fine estimates for BSDEs to prove the consistency of the scheme including the strong boundary conditions (see Lemmas 4.7 and 4.8). We prove the interesting side result that the approximation preserves the property of the original problem to be increasing in the probability variable (see Lemma 4.5).

Our second main contribution is to design the monotone scheme in step 3 and to prove its convergence. Monotone schemes for two-dimensional problems are necessarily nonlocal in the general case, i.e., do not use the values from neighboring mesh points only. The analysis in [37] shows that for correlations approaching \pm 1 and increasing anisotropy, the distance to the furthest mesh points required in terms of the number of cells is unbounded. The present problem poses a worst case in this sense as the correlation is exactly 1 and the anisotropy possibly infinite. Typical monotone schemes are complicated to implement, especially on bounded domains, and necessarily of lower order. In the class of monotone schemes fall the wide stencil schemes in [32], semi-Lagrangian schemes in [22], and generalized finite difference schemes in [7]. They are by construction nonlocal in the above sense and first order accurate only, even for smooth solutions. For bounded domains, such as here, the nature of the wide stencil requires a modification of the schemes near boundaries to avoid overstepping outside the computational mesh (see [39] and [34]). This negatively impacts the consistency of the scheme, and the truncation of the scheme near boundaries necessitates very small time steps for stable solutions. The efficient numerical solution of the discretized nonlinear equations in the implicit case requires heavy machinery (see also [39]) and is still expensive.

On the other hand, standard finite difference discretizations for two-dimensional parabolic equations such as schemes using the nine-point stencil for the cross derivative are nonmonotone. In practice, it is often observed that nonmonotone schemes produce accurate solutions for nonlinear problems in spite of the lack of a theoretical guarantee (see, e.g., [6]). Specifically, there are not many examples in the literature which show that nonmonotone schemes fail for nonlinear second order parabolic equations. We are only aware of [36] who show that a nonmonotone time stepping scheme - the Crank-Nicolson scheme-produces very different approximations in an uncertain volatility model than a monotone scheme. As the latter is proved to converge, this indicates a problem with the former. For the quantile hedging problem, we demonstrate in the numerical section 3.6 that such naive approximations with nonmonotone spatial stencils are not generally positive, and the mathematical property that $p \rightarrow v(0, x, p)$ is nondecreasing on $[0,1]$ does not carry over to the numerical solution. This violates basics properties of the quantile hedging price. This is the first test we are aware of in which a consistent and apparently stable scheme using a nonmonotone spatial approximation gives unphysical solutions. 
The present approach exploits the reduced rank of the diffusion matrix by a suitable mesh construction and enables us to take advantage of the fact that the diffusion acts on lowerdimensional manifolds, here straight lines with different slopes depending on the control. This allows a second order accurate approximation of the diffusion term along these lines with narrow stencils, thus avoiding all the disadvantages of the wide stencil approaches. As these lines are not necessarily aligned with the directions of the drift, the problem does not truly decouple into lower-dimensional problems. However, by choosing a mixed implicit-explicit scheme where the drift is treated explicitly and the diffusion implicitly, hence bypassing strong restrictions on the time step which would result from an explicit treatment of the diffusion term, the discrete scheme does decouple into smaller systems along diagonals in the mesh for each time step. These could be solved in parallel, giving a further computational advantage.

Potential other applications include partial information control problems, including optimal stopping problems; see, e.g., [26], where the filter process has perfect correlation with the original process. These problems therefore share some of the same difficulties as here.

The main challenges in the analysis here come from the nonlinearity of the new term - the driver of the BSDE - in the gradient, the degeneracy of the diffusion operator due to the two state processes being driven by a single Brownian motion, and again the boundedness of the domain in the probability direction. A careful and nonstandard analysis of the consistency of our schemes is needed. In the interior of the domain, we obtain Taylor-like expansions for the solution of the scheme to deduce consistency. Fine BSDE estimates are required in the semidiscrete case, while a generalization of arguments in [3, section 5.2] is needed in the fully discrete case. Near the boundary, the comparison theorems for both the PDEs and finite difference schemes provide upper and lower bounds on the solutions, which allow us to obtain consistency with the boundary conditions in a strong sense. This strategy avoids the use of a Lipschitz property - deduced in previous works from the assumption of a barrier function (see, e.g., [17]) — which is not established for our application. Previous results for PCPT schemes specifically only treat the $\mathbb{R}^{n}$ case (see, e.g., [3]).

The rest of the paper is organized as follows. In section 2 , we give the control approximation (see subsection 2.2), the PCPT scheme (see subsection 2.3), and the monotone finite difference approximations (see subsection 2.4), together with the main results. We also explain in subsection 2.5 how to slightly modify the scheme to relax the compatibility conditions on the discretization parameters. Using this relaxation, we show in subsection 2.6 that some nonmonotone schemes, using cubic spline interpolators as introduced in [24], converge. In section 3 , we present numerical results for a specific application and analyze the observed convergence. The proofs regarding the convergence of the numerical schemes introduced above are postponed to section 4 .

Notations.

- We denote by $\mathcal{M}_{d}(\mathbb{R})$ the set of $d \times d$ matrices with real entries, and $\mathbb{S}^{d}$ is the subset of symmetric matrices.

- For $x \in \mathbb{R}^{d}, \operatorname{diag}(x)$ is the diagonal matrix of size $d$, whose diagonal is given by $x$.

- We denote by $\mathcal{S}$ the sphere in $\mathbb{R}^{d+1}$ of radius 1 and by $\mathcal{D}$ the set of vectors $\eta \in \mathcal{S}$ such that their first component $\eta^{1}=0$. For any $\eta \in \mathcal{S} \backslash \mathcal{D}$, we denote $\eta^{b}:=\frac{1}{\eta^{1}}\left(\eta^{2}, \ldots, \eta^{d+1}\right)^{\top} \in \mathbb{R}^{d}$. By extension, for $\mathcal{Z} \subset \mathcal{S} \backslash \mathcal{D}, \mathcal{Z}^{b}:=\left\{\eta^{b} \in \mathbb{R}^{d} \mid \eta \in \mathcal{Z}\right\}$. 
- For any filtered space $(\Omega, \mathcal{F}, \mathbb{F}, \mathbb{P}), \mathbb{H}^{2}$ is the set of $\mathbb{F}$-predictable processes $\phi$ such that $\mathbb{E}\left[\int_{0}^{T}\left|\phi_{t}\right|^{2} \mathrm{~d} t\right]<+\infty$.

- We further denote by $\mathcal{B C}:=L^{\infty}\left([0, T], \mathcal{C}^{0}\left(\mathbb{R}^{d} \times[0,1]\right)\right)$, the space of functions $u$ that are essentially bounded in time and continuous with respect to their space variable. The convergence in $\mathcal{C}^{0}\left([0, T] \times \mathbb{R}^{d} \times[0,1]\right)$ considered here is local uniform convergence.

2. Numerical schemes and convergence results. We introduce in this section the framework in which we study the quantile hedging problem, and the numerical schemes for its approximation. A first approximation is obtained by control space discretization. We then introduce PCPT schemes for which we prove convergence under some compatibility conditions for the discretization parameters. Last, we relax these conditions, which allows us to prove convergence for some nonmonotone schemes using cubic spline interpolators.

2.1. The quantile hedging problem. On a complete probability space $(\Omega, \mathcal{F}, \mathbb{P})$, we consider a $d$-dimensional Brownian motion $\left(W_{t}\right)_{t \in[0, T]}$ and denote by $\left(\mathcal{F}_{t}\right)_{t \in[0, T]}$ its natural augmented filtration. We suppose that all the randomness comes from the Brownian motion and assume that $\mathcal{F}=\mathcal{F}_{T}$ for some finite terminal time $T>0$.

Let $\mu: \mathbb{R}^{d} \rightarrow \mathbb{R}^{d}, \sigma: \mathbb{R}^{d} \rightarrow \mathcal{M}_{d}(\mathbb{R}), f:[0, T] \times \mathbb{R}^{d} \times \mathbb{R} \times \mathbb{R}^{d} \rightarrow \mathbb{R}$, and $g: \mathbb{R}^{d} \rightarrow \mathbb{R}^{+}$be measurable functions. For any $(t, x, y) \in[0, T] \times \mathbb{R}^{d} \times \mathbb{R}$ and any $\mathbb{R}^{d}$-valued process $\nu \in \mathbb{H}^{2}$, we consider the solution $\left(X^{t, x}, Y^{t, x, y, \nu}\right)$ to the following stochastic differential equations:

$$
\begin{aligned}
& X_{s}=x+\int_{t}^{s} \mu\left(X_{u}\right) \mathrm{d} u+\int_{t}^{s} \sigma\left(X_{u}\right) \mathrm{d} W_{u}, \\
& Y_{s}=y-\int_{t}^{s} f\left(u, X_{u}, Y_{u}, \nu_{u}\right) \mathrm{d} u+\int_{t}^{s} \nu_{u} \mathrm{~d} W_{u}, \quad s \in[t, T] .
\end{aligned}
$$

We will work under the following assumption:

$(H)(\mathrm{i})$ The functions $\mu, \sigma$ are $L$-Lipschitz-continuous and $g$ is bounded and $L_{g}$-Lipschitz, for some nonnegative constants $L, L_{g}$.

(ii) For all $t \in[0, T], f(t, \cdot, \cdot, \cdot)$ is $L$-Lipschitz. For all $(t, x, z) \in[0, T] \times \mathbb{R}^{d} \times \mathbb{R}^{d}$, the function $y \mapsto f(t, x, y, z)$ is decreasing. Moreover, for all $(t, x) \in[0, T] \times \mathbb{R}^{d}$,

$$
f(t, x, 0,0)=0 .
$$

(iii) The function $\sigma$ is invertible and $x \mapsto \sigma^{-1}(x)$ is bounded and Lipschitz-continuous.

In the financial applications we are considering, $X$ will typically represent the log-price of risky assets, the control process $\nu$ is the amount invested in the risky assets, and the function $f$ is nonlinear to allow the inclusion of market frictions in the model.

Remark 2.1. (i) In $(H)($ ii), the monotonicity assumption is not an additional restriction, as in a Lipschitz framework $(H)(\mathrm{i})$, the classical transformation $\tilde{v}(t, x, p):=e^{\lambda t} v(t, x, p)$ for $\lambda$ large enough allows us to reach this setting; see [12, Remark 3.3] for details.

(ii) The condition (2.1) is a reasonable financial modeling assumption: it says that starting out in the market with zero initial wealth and making no investments will lead to a zero value of the wealth process.

(iii) Since $f$ is decreasing and $g$ is bounded, it is easy to see that $|V|_{\infty} \leq|g|_{\infty}$, where $V$ is the superreplication price. 
(iv) Assumption $(H)$ (iii) is required to obtain the PDE characterization of the quantile hedging price [10] and to apply the comparison result from [12].

The quantile hedging problem corresponds to the following stochastic control problem: for $(t, x, p) \in[0, T] \times \mathbb{R}^{d} \times[0,1]$, find

$$
v(t, x, p):=\inf \left\{y \geq 0: \exists \nu \in \mathbb{H}_{2}, \mathbb{P}\left(Y_{T}^{t, x, y, \nu} \geq g\left(X_{T}^{t, x}\right)\right) \geq p\right\} .
$$

Note that we consider admissible strategies valued in the whole $\mathbb{R}^{d}$, i.e., no portfolio constraints.

A key point in the derivation of this PDE is to reformulate the problem as a stochastic target problem by introducing a new control process representing the conditional probability of success. To this end, for any $\mathbb{R}^{d}$-valued process $\alpha \in \mathbb{H}^{2}$, we denote

$$
P_{s}^{t, p, \alpha}:=p+\int_{t}^{s} \alpha_{s} \mathrm{~d} W_{s}, t \leq s \leq T,
$$

and by $\mathcal{A}^{t, p}$ the set of $\alpha \in \mathbb{H}^{2}$ such that $P^{t, p, \alpha} \in[0,1] \mathrm{d} s \otimes \mathrm{d} \mathbb{P}$-almost surely. The problem (2.2) can be rewritten as

$$
v(t, x, p):=\inf \left\{y \geq 0: \exists(\nu, \alpha) \in \mathbb{H}_{2} \times \mathcal{A}^{t, p}, Y_{T}^{t, x, y, \nu} \geq g\left(X_{T}^{t, x}\right) \mathbf{1}_{\left\{P_{T}^{t, p, \alpha}>0\right\}}\right\}
$$

(see [10, Proposition 3.1] for details). In our framework, the above singular stochastic control problem admits a representation in terms of a nonlinear expectation, generated by a BSDE,

$$
v(t, x, p)=\inf _{\alpha \in \mathcal{A}^{t, p}} \mathcal{Y}_{t}^{\alpha}
$$

where $\left(\mathcal{Y}^{\alpha}, \mathcal{Z}^{\alpha}\right)$ is the solution to

$$
\mathcal{Y}_{s}^{\alpha}=g\left(X_{T}^{t, x}\right) \mathbf{1}_{\left\{P_{T}^{t, p, \alpha}>0\right\}}+\int_{s}^{T} f\left(s, X_{s}, \mathcal{Y}_{s}^{\alpha}, \mathcal{Z}_{s}^{\alpha}\right) \mathrm{d} s-\int_{s}^{T} \mathcal{Z}_{s}^{\alpha} \mathrm{d} W_{s}, t \leq s \leq T .
$$

The results in [9] justify the previous representation and give a dynamic programming principle for the control problem in a general setting.

Following [5], it has been shown in [12] that the function $v$ is equivalently a viscosity solution in $[0, T) \times \mathbb{R}^{d} \times(0,1)$ of the following PDE (see [12, Theorem 3.1]):

$$
\mathcal{H}\left(t, x, \varphi, \partial_{t} \varphi, D \varphi, D^{2} \varphi\right)=0,
$$

where $\mathcal{H}$ is the following continuous operator, for which a comparison theorem holds (see [12, Theorem 3.2]): for $\Theta:=(t, x, y, b, q, A)$ with $(t, x, y, b) \in[0, T] \times \mathbb{R}^{d} \times \mathbb{R}^{+} \times \mathbb{R}, q:=\left(\begin{array}{c}q^{x} \\ q^{p}\end{array}\right) \in \mathbb{R}^{d+1}$, and $A:=\left(\begin{array}{cc}A^{x x} & A^{x p} \\ A^{x p} & A^{p p}\end{array}\right) \in \mathbb{S}^{d+1}$ (here $\left(q^{x}, q^{p}\right) \in \mathbb{R}^{d} \times \mathbb{R}$ and $\left(A^{x x}, A^{x p}, A^{p p}\right) \in \mathbb{S}^{d} \times \mathbb{R}^{d} \times \mathbb{R}$, where an element of $\mathbb{R}^{d}$ is a column vector),

$$
\mathcal{H}(\Theta)=\sup _{\eta \in \mathcal{S} \backslash \mathcal{D}} H^{\eta}(\Theta)
$$

Copyright $\odot$ by SIAM. Unauthorized reproduction of this article is prohibited. 
with

$$
H^{\eta}(\Theta):=\left(\eta^{1}\right)^{2}\left(-b-f\left(t, x, y, \mathfrak{z}\left(x, q, \eta^{b}\right)\right)-\mathcal{L}\left(x, q, A, \eta^{b}\right)\right) \quad \text { for } \eta \in \mathcal{S} \backslash \mathcal{D}
$$

and

$$
\begin{aligned}
\mathfrak{z}(x, q, a) & :=q^{x} \sigma(x)+q^{p} a, \\
\mathcal{L}(x, q, A, a) & :=\mu(x)^{\top} q^{x}+\frac{1}{2} \operatorname{Tr}\left[\sigma(x) \sigma(x)^{\top} A^{x x}\right]+\frac{|a|^{2}}{2} A^{p p}+a^{\top} \sigma(x)^{\top} A^{x p} .
\end{aligned}
$$

Remark 2.2. Under $(H)$, the mapping $\mathcal{S} \backslash \mathcal{D} \ni \eta \mapsto H^{\eta}(\Theta) \in \mathbb{R}$ extends continuously to $\mathcal{S}$ by setting (see [12, Remark 3.1]), for all $\eta \in \mathcal{D}$,

$$
H^{\eta}(\Theta)=-\frac{1}{2} A^{p p}
$$

and $\mathcal{H}(\Theta)=\sup _{\eta \in \mathcal{S}} H^{\eta}(\Theta)$.

In addition, the value function $v$ continuously satisfies the following boundary conditions in the $p$-variable:

$$
v(t, x, 0)=0 \text { and } v(t, x, 1)=V(t, x) \text { on }[0, T] \times \mathbb{R}^{d},
$$

where $V$ is the superreplication price of the contingent claim with payoff $g(\cdot)$. It is also known that $v$ has a discontinuity as $t \rightarrow T$. By definition, the terminal condition is

$$
\mathbb{R}^{d} \times[0,1] \ni(x, p) \mapsto g(x) 1_{p>0} \in \mathbb{R}^{+},
$$

but the values which are continuously attained are obtained by convexification [10]:

$$
v(T-, x, p)=p g(x) \text { on } \mathbb{R}^{d} \times[0,1],
$$

and we shall work with this terminal condition at $t=T$ from now on.

2.2. Discrete set of controls. We start by discretizing the set of controls $\mathcal{S}$. Let $\left(\mathcal{R}_{n}\right)_{n \geq 1}$ be an increasing sequence of closed subsets of $\mathcal{S} \backslash \mathcal{D}$ such that

$$
\overline{\bigcup_{n \geq 1} \mathcal{R}_{n}}=\mathcal{S}
$$

For $n \geq 1$, let $v_{n}:[0, T] \times \mathbb{R}^{d} \times[0,1] \rightarrow \mathbb{R}$ be the unique continuous viscosity solution of

$$
\mathcal{H}_{n}\left(t, x, \varphi, \partial_{t} \varphi, D \varphi, D^{2} \varphi\right)=0, \quad(t, x, p) \in[0, T) \times \mathbb{R}^{d} \times(0,1),
$$

subject to (2.4)-(2.5) - see Corollary 4.2-where the operator $\mathcal{H}_{n}$ is naturally given by

$$
\mathcal{H}_{n}(\Theta):=\sup _{\eta \in \mathcal{R}_{n}} H^{\eta}(\Theta)
$$

Proposition 2.3. Under Assumption (H), $v_{n} \rightarrow v$ in $\mathcal{C}^{0}\left([0, T] \times \mathbb{R}^{d} \times[0,1]\right)$. 
2.3. The discrete-time PCPT scheme. From now on, we fix $n \geq 1$ and $\mathcal{R}_{n}$ as the associated discrete set of control. For $(t, x, y) \in[0, T] \times \mathbb{R}^{d} \times \mathbb{R}^{+}, q \in \mathbb{R}^{d+1}$, and $A \in \mathbb{S}^{d+1}$, denoting $\Xi:=(t, x, y, q, A)$, we define

$$
\mathcal{F}_{n}(\Xi)=\sup _{a \in \mathcal{R}_{n}^{b}} F^{a}(\Xi) \text { with } F^{a}(\Xi):=-f(t, x, y, \mathfrak{z}(x, q, a))-\mathcal{L}(x, q, A, a),
$$

where $\mathfrak{z}$ and $\mathcal{L}$ are defined above Remark 2.2. By Corollary 4.2 , we easily observe that $v_{n}$ is also the unique viscosity solution to

$$
-\partial_{t} \varphi+\mathcal{F}_{n}\left(t, x, \varphi, D \varphi, D^{2} \varphi\right)=0 \text { on }[0, T) \times \mathbb{R}^{d} \times(0,1)
$$

with the boundary conditions (2.4) and (2.5). Let us observe in particular that $K:=\mathcal{R}_{n}^{b}$ is a discrete subset of $\mathbb{R}^{d}$ and that the PDE (2.8) is written in a more classical way. We will mainly consider this form in the following, as it is simpler to study.

To approximate $v_{n}$, we consider an adaptation of the PCPT scheme in [31, 3, 38], and especially [20], to our setting, as described below.

Definition 2.4 (solution $S^{a}(\cdot)$ ). For $0 \leq t<s \leq T, a \in K$, and a continuous $\phi: \mathbb{R}^{d} \times$ $[0,1] \rightarrow \mathbb{R}$, we denote by $S^{a}(t, s, \phi):[t, s] \times \mathbb{R}^{d} \times[0,1] \rightarrow \mathbb{R}$ the unique solution of

$$
\begin{aligned}
-\partial_{t} \varphi+F^{a}\left(r, x, \varphi, D \varphi, D^{2} \varphi\right)=0 & \text { on }[t, s) \times \mathbb{R}^{d} \times(0,1), \\
\varphi(s, x, p)=\phi(x, p) & \text { on } \mathbb{R}^{d} \times[0,1], \\
\varphi(r, x, p)=B^{p}(t, s, \phi)(r, x) & \text { on }[t, s) \times \mathbb{R}^{d} \times\{0,1\} .
\end{aligned}
$$

Here, for $p \in\{0,1\}, B^{p}(t, s, \phi)$ is the solution to

$$
-\partial_{t} \varphi+F^{0}\left(r, x, \varphi, D \varphi, D^{2} \varphi\right)=0 \text { on }[t, s) \times \mathbb{R}^{d}
$$

with terminal condition $B^{p}(t, s, \phi)(s, x)=\phi(x, p)$.

For $\kappa \geq 1$, we consider a grid on the time interval $[0, T]$,

$$
\pi=\left\{0=: t_{0}<\cdots<t_{j}<\cdots<t_{\kappa}:=T\right\},
$$

and denote $|\pi|:=\max _{0 \leq j<\kappa}\left(t_{j+1}-t_{j}\right)$. For any $t \in[0, T]$, we define

$$
t_{\pi}^{+}:=\inf \{r \in \pi \mid r>t\} \text { and } t_{\pi}^{-}:=\sup \{r \in \pi \mid r \leq t\} .
$$

We will drop the subscript $\pi$ for brevity whenever we consider a fixed mesh.

Definition 2.5 (solution to the semidiscrete PCPT scheme). The solution to the semidiscrete PCPT scheme associated with the grid $\pi$ is defined as the function $v_{n, \pi}:[0, T] \times \mathbb{R}^{d} \times[0,1] \rightarrow \mathbb{R}$ such that

$$
\mathfrak{S}\left(\pi, t, x, p, v_{n, \pi}(t, x, p), v_{n, \pi}\right)=0,
$$

where, for a grid $\pi,(t, x, p, y) \in[0, T] \times \mathbb{R}^{d} \times[0,1] \times \mathbb{R}^{+}$and a function $u \in \mathcal{B C}$,

$$
\mathfrak{S}(\pi, t, x, p, y, u)=\left\{\begin{aligned}
y-\min _{a \in K} S^{a}\left(t_{\pi}^{-}, t_{\pi}^{+}, u\left(t_{\pi}^{+}, \cdot\right)\right)(t, x, p) & \text { if } t<T, \\
y-g(x) p & \text { otherwise. }
\end{aligned}\right.
$$

Copyright (C) by SIAM. Unauthorized reproduction of this article is prohibited. 
Let us observe that the function $v_{n, \pi}$ can alternatively, and perhaps more intuitively, be described by the following backward algorithm:

1. Initialization: set $v_{n, \pi}(T, x, p):=g(x) p$ for $(x, p) \in \mathbb{R}^{d} \times[0,1]$.

2. Backward step: for $j=\kappa-1, \ldots, 0$, compute $w^{j, a}:=S^{a}\left(t_{j}, t_{j+1}, v_{n, \pi}\left(t_{j+1}, \cdot\right)\right)$ and set

$$
v_{n, \pi}:=\min _{a \in K} w^{j, a} \text { on }\left[t_{j}, t_{j+1}\right) \times \mathbb{R}^{d} \times[0,1] .
$$

Remark 2.6. In our setting, we can easily identify the boundary values (of the scheme):

1. At $p=0$, the terminal condition is $\phi(T, x)=0\left(\right.$ recall that $\left.v(T, x, p)=g(x) \mathbf{1}_{\{p>0\}}\right)$, and this propagates through the backward iteration, so that $v_{n, \pi}(t, x, 0)=0$ for all $(t, x) \in[0, T] \times \mathbb{R}^{d}$.

2. At $p=1$, the terminal condition is $\phi(T, x)=g(x)$ and the boundary condition is thus given by $v_{n, \pi}(t, x, 1)=V(t, x)$ for all $(t, x) \in[0, T] \times \mathbb{R}^{d}$, where $V$ is the superreplication price.

The main result of this section is the following.

Theorem 2.7. Under Assumption (H), $v_{n, \pi} \rightarrow v_{n}$ in $\mathcal{C}^{0}\left([0, T] \times \mathbb{R}^{d} \times[0,1]\right)$ as $|\pi| \rightarrow 0$.

To conclude this section, let us observe that we obtain the following result, combining Proposition 2.3 and Theorem 2.7.

Corollary 2.8. Under Assumption (H), we have in $\mathcal{C}^{0}\left([0, T] \times \mathbb{R}^{d} \times[0,1]\right)$,

$$
\lim _{n \rightarrow \infty} \lim _{|\pi| \downarrow 0} v_{n, \pi}=v
$$

Remark 2.9. An important question, from a numerical perspective, is to understand how to fix the parameters $n$ and $\pi$ in relation to each other. The theoretical difficulty here is to obtain a precise rate of convergence for the approximations given in Proposition 2.3 and Theorem 2.7, along the lines of the continuous dependence estimates with respect to the control discretization in $[28,20]$, and estimates of the approximation by piecewise constant controls as in [30, 29]. To answer this question in our general setting is a challenging task, extending also to error estimates for the full discretization in the next section, which is left for further research.

2.4. Fully discrete monotone scheme in a one-dimensional Brownian setting. The goal of this section is to introduce a fully implementable scheme, obtained by adding a finite difference approximation to the PCPT procedure described in section 2.3. Then in section 3, we present numerical tests that demonstrate the practical feasibility of our numerical method.

From now on, we will assume that the log-price process $X$ is a one-dimensional Brownian motion with drift for $(t, x) \in[0, T] \times \mathbb{R}$ :

$$
X_{s}^{t, x}=x+\mu(s-t)+\sigma\left(W_{s}-W_{t}\right), \quad s \in[t, T],
$$

with $\mu \in \mathbb{R}$ and $\sigma>0$. This restriction to the Black-Scholes model is not essential, as the main difficulty and nonlinearities are already present in this case and the analysis technique can be extended straightforwardly to more general monotone schemes in the setting of more complex

Copyright $\odot$ by SIAM. Unauthorized reproduction of this article is prohibited. 
SDEs for $X$. We take advantage of the specific dynamics to design a simple to implement numerical scheme, which also simplifies the notation.

We shall moreover work under the following hypothesis.

Hypothesis 2.10. The coefficient $\mu$ is nonnegative.

Remark 2.11. This assumption is introduced without loss of generality in order to simplify the notation. We detail in Remark 2.13(i) how to modify the scheme for nonpositive drift $\mu$. The convergence properties are the same.

We now fix $n \geq 1$ and $K:=\mathcal{R}_{n}^{b}$ is the associated discrete set of controls (see section 2.2), assuming that $0 \notin K$ and recalling that $v_{n}$ is the solution to (2.8). For $\kappa \geq 1$, we consider the grid $\pi=\left\{0=: t_{0}<\cdots<t_{j}<\cdots<t_{\kappa}:=T\right\}$ on $[0, T]$ and approximate $v_{n}$ by a PCPT scheme, extending section 2.3. The main point here is that we introduce a finite difference approximation for the solution $S^{a}(\cdot), a \in K$, to (2.9)-(2.11). This approximation, denoted by $S_{\delta}^{a}(\cdot)$ for a discretization parameter $\delta>0$, is specified in Definition 2.20 below. Each approximation $S_{\delta}^{a}(\cdot)$ is defined on a spatial grid

$$
\mathcal{G}_{\delta}^{a}:=\delta \mathbb{Z} \times \Gamma_{\delta}^{a} \subset \mathbb{R} \times[0,1],
$$

where $\Gamma_{\delta}^{a}$ is a uniform grid on $[0,1]$ with $N_{\delta}^{a}+1$ points and mesh size $1 / N_{\delta}^{a}$, which we define now.

First, we observe that for the model of this section, (2.8) can be rewritten as

$$
\sup _{a \in K}\left(-D^{a} \varphi-\mu \nabla^{a} \varphi-\frac{\sigma^{2}}{2} \Delta^{a} \varphi-f\left(t, x, \varphi, \sigma \nabla^{a} \varphi\right)\right)=0,
$$

with

$$
\nabla^{a} \varphi:=\partial_{y} \varphi+\frac{a}{\sigma} \partial_{p} \varphi, \quad \Delta^{a} \varphi:=\partial_{y y}^{2} \varphi+2 \frac{a}{\sigma} \partial_{y p}^{2} \varphi+\frac{a^{2}}{\sigma^{2}} \partial_{p p}^{2} \varphi, \quad D^{a} \varphi:=\partial_{t} \varphi-\frac{a}{\sigma} \mu \partial_{p} \varphi .
$$

Exploiting the degeneracy of the operators $\nabla^{a}$ and $\Delta^{a}$ in the direction $(a,-\sigma)$, we construct $\Gamma_{\delta}^{a}$ so that the solution to (2.19) is approximated by the solution of an implicit finite difference scheme with only one-directional derivatives. To take into account the boundaries $p=0, p=1$, we define (recall that $a \neq 0$ )

$$
N_{\delta}^{a}:=\min \left\{l \geq 1: l \frac{|a|}{\sigma} \delta \geq 1\right\}=\left\lceil\frac{\sigma}{|a| \delta}\right\rceil=: \frac{\sigma}{|\mathfrak{a}(a, \delta)| \delta}, \quad \text { where } \mathfrak{a}(a, \delta):=\operatorname{sgn}(a) \frac{\sigma}{\delta N_{\delta}^{a}} .
$$

We finally set

$$
\Gamma_{\delta}^{a}:=\left\{0, \frac{|\mathfrak{a}(a, \delta)|}{\sigma} \delta, \ldots, N_{\delta}^{a} \frac{|\mathfrak{a}(a, \delta)|}{\sigma} \delta=1\right\}=\left\{\frac{l}{N_{\delta}^{a}}: l=0, \ldots, N_{\delta}^{a}\right\} .
$$

We have then defined $\mathcal{G}_{\delta}^{a}=\left\{\left(k \delta, \frac{l}{N_{\delta}^{a}}\right) \mid k \in \mathbb{Z}, 0 \leq l \leq N_{\delta}^{a}\right\}=:\left\{\left(x_{k}, p_{l}\right) \mid k \in \mathbb{Z}, 0 \leq\right.$ $\left.l \leq N_{\delta}^{a}\right\}$ using (2.18). An element of $\ell^{\infty}\left(\mathcal{G}_{\delta}^{a}\right)$ is denoted by $u=\left(u_{k, l}\right)_{k \in \delta \mathbb{Z}, 0 \leq l \leq N_{\delta}^{a}}:=$ $\left(u\left(x_{k}, p_{l}\right)\right)_{k \in \delta \mathbb{Z}, 0 \leq l \leq N_{\delta}^{a}}$. 
We now introduce the finite difference operators needed to define $S_{\delta}^{a}(\cdot)$, and we construct $S_{\delta}^{a}(\cdot)$ such that $S_{\delta}^{a}(t, s, \varphi) \in \ell\left(\mathcal{G}_{\delta}^{a}\right)$ for any $a \in K$, any $0 \leq t<s \leq T$, and any bounded map $\varphi: \delta \mathbb{Z} \times[0,1] \rightarrow \mathbb{R} ;$ see Proposition 2.15 .

Using the degeneracy of $\nabla^{\mathfrak{a}(a, \delta)}$ and $\Delta^{\mathfrak{a}(a, \delta)}$ in the direction $(\mathfrak{a}(a, \delta),-\sigma)$, we define the following finite difference operators for $v=\left(v_{k, l}\right)_{k \in \mathbb{Z}, 0 \leq l \leq N_{\delta}^{a}}=\left(v\left(x_{k}, p_{l}\right)\right)_{k \in \mathbb{Z}, 0 \leq l \leq N_{\delta}^{a}} \in \ell^{\infty}\left(\mathcal{G}_{\delta}^{a}\right)$ and $w=\left(w_{k}\right)_{k \in \mathbb{Z}} \in \ell^{\infty}(\delta \mathbb{Z})$ :

$$
\begin{aligned}
\nabla_{\delta}^{a} v_{k, l}:=\frac{1}{2 \delta}\left(v_{k+1, l+\operatorname{sgn}(a)}-v_{k-1, l-\operatorname{sgn}(a)}\right), & \nabla_{\delta} w_{k}:=\frac{1}{2 \delta}\left(w_{k+1}-w_{k-1}\right), \\
\nabla_{+, \delta}^{a} v_{k, l}:=\frac{1}{\delta}\left(v_{k+1, l+\operatorname{sgn}(a)}-v_{k, l}\right), & \nabla_{+, \delta} w_{k}:=\frac{1}{\delta}\left(w_{k+1}-w_{k}\right), \\
\Delta_{\delta}^{a} v_{k, l}:=\frac{1}{\delta^{2}}\left(v_{k+1, l+\operatorname{sgn}(a)}+v_{k-1, l-\operatorname{sgn}(a)}-2 v_{k, l}\right), & \Delta_{\delta} w_{k}:=\frac{1}{\delta^{2}}\left(w_{k+1}+w_{k-1}-2 w_{k}\right) .
\end{aligned}
$$

Let $\theta>0$ be a parameter to be fixed later. We define, for $\left(t, x, y, q, q_{+}, A\right) \in[0, T] \times \mathbb{R}^{5}$,

$$
\begin{aligned}
F(t, x, y, q, A) & :=-\mu q-\frac{\sigma^{2}}{2} A-f(t, x, y, \sigma q) \text { and } \\
\widehat{F}\left(t, x, y, q, q_{+}, A\right) & :=-\mu q_{+}-\left(\frac{\sigma^{2}}{2}+\theta \frac{\delta^{2}}{h}\right) A-f(t, x, y, \sigma q) .
\end{aligned}
$$

Definition 2.12 (discrete solution $S_{\delta}^{a}(\cdot)$ ). We fix $a \in K, \delta>0,0 \leq t<s \leq T$, and $h:=s-t>0$.

1. Boundary operator. Let $\varphi: \delta \mathbb{Z} \rightarrow \mathbb{R}$. We define $B_{\delta}^{a}(t, s, \varphi) \in \ell^{\infty}(\delta \mathbb{Z})$ as the unique solution $v \in \ell^{\infty}(\delta \mathbb{Z})$ to (see Proposition (4.10) for the well-posedness of this definition)

$$
S_{b}\left(k, v_{k}, \nabla_{\delta} v_{k}, \nabla_{+, \delta} v_{k}, \Delta_{\delta} v_{k}, \varphi\right)=0, \quad k \in \mathbb{Z},
$$

where, for any $k \in \mathbb{Z},\left(y, q, q_{+}, A\right) \in \mathbb{R}^{4}$ and $u \in \ell^{\infty}(\delta \mathbb{Z})$,

$$
S_{b}\left(k, y, q, q_{+}, A, u\right)=y-u_{k}+h \widehat{F}\left(t, k \delta, y, q, q_{+}, A\right) .
$$

2. Let $\varphi: \delta \mathbb{Z} \times[0,1] \rightarrow \mathbb{R}$. We define $S_{\delta}^{a}(t, s, \varphi) \in \ell^{\infty}\left(\mathcal{G}_{\delta}^{a}\right)$ as the unique solution $v \in \ell^{\infty}\left(\mathcal{G}_{\delta}^{a}\right)$ to (see Proposition (2.15) below for the well-posedness of this definition)

$$
\begin{aligned}
S\left(k, l, v_{k, l}, \nabla_{\delta}^{a} v_{k, l}, \nabla_{+, \delta}^{a} v_{k, l}, \Delta_{\delta}^{a} v_{k, l}, \varphi\right) & =0, \\
v_{k, 0}=\underline{v}_{k}, \quad v_{k, N_{\delta}^{a}} & =\bar{v}_{k},
\end{aligned}
$$

where $\underline{v}:=B_{\delta}^{a}(t, s, \varphi(\cdot, 0)), \bar{v}:=B_{\delta}^{a}(t, s, \varphi(\cdot, 1))$, and where, for any $k \in \mathbb{Z}, 0<l<$ $N_{\delta}^{a},\left(y, q, q_{+}, A\right) \in \mathbb{R}^{4}$, and any bounded $u: \delta \mathbb{Z} \times[0,1] \rightarrow \mathbb{R}$,

$$
S\left(k, l, y, q, q_{+}, A, u\right)=y-u\left(x_{k}, \mathfrak{p}^{a}\left(p_{l}\right)\right)+h \widehat{F}\left(t, k \delta, y, q, q_{+}, A\right),
$$

where we define, for all $p \in[0,1]$,

$$
\mathfrak{p}^{a}(p):=p-\mu \frac{\mathfrak{a}(a, \delta)}{\sigma} h .
$$

Copyright $\odot$ by SIAM. Unauthorized reproduction of this article is prohibited. 
Remark 2.13. (i) Here, as stated before, we have assumed $\mu \geq 0$. If the opposite is true, one has to consider $\nabla_{-, \delta}^{a} v_{k, l}:=\frac{1}{\delta}\left(v_{k, l}-v_{k-1, l-\operatorname{sgn}(a)}\right)\left(\operatorname{resp} ., \nabla_{-, \delta} w_{k}:=\frac{1}{\delta}\left(w_{k}-w_{k-1}\right)\right)$ instead of $\nabla_{+, \delta}^{a} v_{k, l}\left(\operatorname{resp} ., \nabla_{+, \delta} w_{k}\right)$ in the definition of $S_{\delta}^{a}(s, t, \varphi)$ (resp., $\left.\underline{v}_{k}, \bar{v}_{k}\right)$, to obtain a monotone scheme.

(ii) For the nonlinearity $f$, we used the Lax-Friedrichs scheme [15, 20], adding the $\theta\left(v_{+}+v_{-}-2 v\right)$ term in the definition of $\widehat{F}$ to enforce monotonicity.

Let $0 \leq t<s \leq T, \delta>0, \varphi: \delta \mathbb{Z} \times[0,1] \rightarrow \mathbb{R}$, and $h:=s-t>0$. We now assume that the following conditions on the parameters are satisfied:

$$
\begin{aligned}
\delta & \leq 1, \\
\frac{h L}{2 \delta} \leq \theta & <\frac{1}{4}, \\
\mu h \leq \delta & \leq M h
\end{aligned}
$$

for a constant $M>0$ independent of $h$ and $\delta$.

Remark 2.14. Since $\mu h \leq \delta$, we have $\left|\mu \frac{\mathfrak{a}(a, \delta)}{\sigma} h\right| \leq \frac{|\mathfrak{a}(a, \delta)|}{\sigma} \delta$, which ensures that from (2.29), $\mathfrak{p}^{a}\left(p_{l}\right) \in[0,1]$ for all $0<l<N_{\delta}^{a}$.

Under these conditions, we prove that $S_{\delta}^{a}(t, s, \varphi)$ is uniquely defined for any bounded terminal condition $\varphi$, and that it can be obtained by Picard iteration. Moreover, strong uniqueness is guaranteed by a comparison theorem. We refer to subsection 4.3 for the proofs.

Proposition 2.15. Assume (2.30)-(2.32) in addition to Assumptions (H) and 2.10. For every bounded function $\varphi: \delta \mathbb{Z} \times[0,1] \rightarrow \mathbb{R}$, there exists a unique solution in $\ell^{\infty}\left(\mathcal{G}_{\delta}^{a}\right)$ to $(2.26)-(2.27)$.

Proposition 2.16. Assume (2.30)-(2.32) in addition to Assumptions (H) and 2.10. Let $\varphi^{1}, \varphi^{2}: \delta \mathbb{Z} \times[0,1] \rightarrow \mathbb{R}$ be two bounded functions satisfying $\varphi^{1} \leq \varphi^{2}$ on $\delta \mathbb{Z} \times[0,1]$.

1. (monotonicity) For all $k \in \mathbb{Z}, 1 \leq l \leq N_{a},\left(v, q, q_{+}, A\right) \in \mathbb{R}^{4}$, we have

$$
S\left(k, l, v, q, q_{+}, A, \varphi^{2}\right) \leq S\left(k, l, v, q, q_{+}, A, \varphi^{1}\right) .
$$

2. (comparison theorem) Let $\left(v^{1}, v^{2}\right) \in \ell^{\infty}\left(\mathcal{G}_{\delta}^{a}\right)^{2}$ such that

$$
\begin{aligned}
S\left(k, l, v_{k, l}^{1}, \nabla_{\delta}^{a} v_{k, l}^{1},\right. & \left.\nabla_{+, \delta}^{a} v_{k, l}^{1}, \Delta_{\delta}^{a} v_{k, l}^{1}, \varphi^{2}\right) \\
& \leq S\left(k, l, v_{k, l}^{2}, \nabla_{\delta}^{a} v_{k, l}^{2}, \nabla_{+, \delta}^{a} v_{k, l}^{2}, \Delta_{\delta}^{a} v_{k, l}^{2}, \varphi^{2}\right), \\
v_{k, 0}^{1} & \leq v_{k, 0}^{2} \\
v_{k, N_{\delta}^{a}}^{1} & \leq v_{k, N_{\delta}^{a}}^{2}
\end{aligned}
$$

for all $k \in \mathbb{Z}$ and $0<l<N_{\delta}^{a}$. Then $v^{1} \leq v^{2}$.

3. We have $S_{\delta}^{a}\left(s, t, \varphi^{1}\right)_{k, l} \leq S_{\delta}^{a}\left(s, t, \varphi^{2}\right)_{k, l}$ for all $k \in \mathbb{Z}$ and $0 \leq l \leq N_{\delta}^{a}$.

We last give a refinement of the comparison theorem which will be useful in what follows.

Proposition 2.17. Assume (2.30)-(2.32) in addition to Assumptions (H) and 2.10. Let $u: \delta \mathbb{Z} \times[0,1] \rightarrow \mathbb{R}$ be a bounded function, and let $v^{1}, v^{2} \in \ell^{\infty}\left(\mathcal{G}_{\delta}^{a}\right)$. Assume that, for all $k \in \mathbb{Z}$ 
and $0<l<N_{\delta}^{a}$, we have

$$
\begin{aligned}
S\left(k, l, v_{k, l}^{1}, \nabla_{\delta}^{a} v_{k, l}^{1},\right. & \left.\nabla_{+, \delta}^{a} v_{k, l}^{1}, \Delta_{\delta}^{a} v_{k, l}^{1}, u\right) \\
& \leq 0 \leq S\left(k, l, v_{k, l}^{2}, \nabla_{\delta}^{a} v_{k, l}^{2}, \nabla_{+, \delta}^{a} v_{k, l}^{2}, \Delta_{\delta}^{a} v_{k, l}^{2}, u\right) .
\end{aligned}
$$

Then

$$
v_{k, l}^{1}-v_{k, l}^{2} \leq e^{-4 \frac{\mathfrak{a}(a, \delta)^{2}}{\sigma^{2}} C(h, \delta) l\left(N_{\delta}^{a}-l\right)}\left(\left|\left(v_{\cdot, 0}^{1}-v_{\cdot, 0}^{2}\right)^{+}\right|_{\infty}+\left|\left(v_{\cdot, N_{\delta}^{a}}^{1}-v_{\cdot, N_{\delta}^{a}}^{2}\right)^{+}\right|_{\infty}\right),
$$

where

$$
C(h, \delta):=\frac{\ln \left(\frac{1+\frac{h}{\delta} \mu+\sigma^{2} \frac{h}{\delta^{2}}+2 \theta+\frac{h L}{2 \delta}}{\frac{h}{\delta} \mu+\sigma^{2} \frac{h}{\delta^{2}}+2 \theta+\frac{h L}{2 \delta}}\right)}{\delta^{2}} .
$$

Moreover,

$$
C(h, \delta) \geq \frac{1}{\left(\left(\mu+\frac{L}{2}\right) M+2 \theta M^{2}\right) h^{2}+\sigma^{2} h}-\frac{M^{2}}{2 \sigma^{4}} .
$$

Remark 2.18. (i) To prove the consistency of the scheme, we define in Lemma 4.17 smooth functions $w^{ \pm}$so that $\left(w^{ \pm}\left(x_{k}, p_{l}\right)\right) \in \ell^{\infty}\left(\mathcal{G}_{\delta}^{a}\right)$ satisfy $S \geq 0$ or $S \leq 0$, but we cannot use the comparison theorem directly, as the values at the boundary cannot be controlled. The previous proposition will be used in Lemma 4.18 to show that the difference between $w^{ \pm}$and the linear interpolant of a solution of $S=0$ is small.

(ii) The coefficient $\exp \left(-4 \frac{\mathfrak{a}(a, \delta)}{\sigma^{2}} C(h, \delta) l\left(N_{\delta}^{a}-l\right)\right.$ that appears in the first equation of the previous proposition shows that the dependence on the boundary values decays exponentially with the distance to the boundary. This was to be expected and was already observed in other situations; see, for example, [2, Theorem 4.3] for the heat equation.

In order to define our approximation of $v_{n}$, it is not enough to replace $S^{a}(\cdot)$ by $S_{\delta}^{a}(\cdot)$ in the minimization (2.15) or, similarly, (2.16), as the approximations are not defined on the same grid for the probability variable. This flexibility of having different grids will be important for the construction of the schemes later on. We thus have to consider a supplementary step which consists in a linear interpolation in the probability variable.

Definition 2.19 (linear interpolation). For any mapping $u \in \ell^{\infty}\left(\mathcal{G}_{\delta}^{a}\right)$, we define its extension $\mathcal{I}_{\delta}^{a}(u): \delta \mathbb{Z} \times[0,1] \rightarrow \mathbb{R}$ by linear interpolation in the second variable. More precisely, if $u \in \ell^{\infty}\left(\mathcal{G}_{\delta}^{a}\right), k \in \mathbb{Z}$ and $p \in\left[p_{l}, p_{l+1}\right)$ with $0 \leq l<N_{\delta}^{a}$,

$$
\mathcal{I}_{\delta}^{a}(u)\left(x_{k}, p\right):=\frac{p_{l+1}-p}{p_{l+1}-p_{l}} u_{k, l}+\frac{p-p_{l}}{p_{l+1}-p_{l}} u_{k, l+1},
$$

and obviously $\mathcal{I}_{\delta}^{a}(u)\left(x_{k}, 1\right):=u_{k, N_{\delta}^{a}}$.

Definition 2.20 (solution to the fully discrete PCPT scheme). The solution to the fully discrete PCPT scheme associated with $\pi, \delta$ is then $v_{n, \pi, \delta}: \pi \times \delta \mathbb{Z} \times[0,1] \rightarrow \mathbb{R}$ satisfying

$$
\widehat{\mathfrak{S}}\left(\pi, \delta, j, k, p, v_{n, \pi, \delta}\left(t_{k}, x_{j}, p\right), v_{n, \pi, \delta}\right)=0,
$$

Copyright (C) by SIAM. Unauthorized reproduction of this article is prohibited. 
where, for any $0 \leq j \leq \kappa, k \in \mathbb{Z}, p \in[0,1], y \in \mathbb{R}^{+}$, and any bounded function $u: \pi \times \delta \mathbb{Z} \times$ $[0,1] \rightarrow \mathbb{R}$

$$
\widehat{\mathfrak{S}}(\pi, \delta, j, k, p, y, u)=\left\{\begin{aligned}
y-\min _{a \in K} \mathcal{I}_{\delta}^{a}\left(S_{\delta}^{a}\left(t_{j}, t_{j+1}, u\left(t_{j+1}, \cdot\right)\right)\right)\left(x_{k}, p\right) & \text { if } j<\kappa, \\
y-g\left(x_{k}\right) p & \text { otherwise. }
\end{aligned}\right.
$$

Alternatively, $v_{n, \pi, \delta}$ is defined by the following backward induction:

1. Initialization: set $v_{n, \pi, \delta}\left(T, x_{k}, p\right):=g\left(x_{k}\right) p$ for $k \in \mathbb{Z}$ and $p \in[0,1]$.

2. Backward step: for $j=\kappa-1, \ldots, 0$, solve the finite difference schemes $w_{\delta}^{j, a}:=S_{\delta}^{a}\left(t_{j}, t_{j+1}, v_{n, \pi, \delta}\left(t_{j+1}, \cdot\right)\right)$ for each $a \in K$ and set, for $k \in \mathbb{Z}$ and $p \in[0,1]$,

$$
v_{n, \pi, \delta}\left(t_{j}, x_{k}, p\right):=\inf _{a \in K} \mathcal{I}_{\delta}^{a}\left(w_{\delta}^{j, a}\right)\left(x_{k}, p\right) .
$$

We now can state the main result of this section.

Theorem 2.21. Under Assumptions ( $H$ ) and 2.10, the function $v_{n, \pi, \delta}$ converges to $v_{n}$ uniformly on compact sets as $|\pi|, \delta \rightarrow 0$, where $\pi=\left\{0=t_{0}<t_{1}<\cdots<t_{\kappa}=T\right\}$ and $\delta$ satisfy conditions (2.30)-(2.32) for all $h=t_{j+1}-t_{j}, 0 \leq j<\kappa$.

We prove in subsection 4.4 that the scheme is monotone (Proposition 4.13), stable (Proposition 4.14), consistent with $(2.8)$ in $[0, T) \times \mathbb{R} \times(0,1)$ (Proposition 4.20) and with the boundary conditions (Proposition 4.12). The theorem then follows by identical arguments to [4].

2.5. Relaxing the conditions. The conditions (2.31) and (2.32) restrict the time step $h$ and spatial mesh size $\delta$ to be proportional to each other. Here, the left-hand inequality in (2.31) is a standard CFL condition needed to ensure the monotonicity and stability of the semi-Lagrangian step $\mathfrak{p}^{a}$ in (2.28); the left-hand inequality in (2.32) is a requirement of the well-posedness of the implict Lax-Friedrichs term; and the right-hand inequality in (2.32), an "inverse CFL condition," is a requirement of consistency for the linear interpolations involved.

In this section, we propose a slightly modified scheme with the goal to remove the first of these conditions, i.e., we replace (2.31) by

$$
\delta \leq M h
$$

for a constant $M$ independent of $h$ and $\delta$.

In addition to the operators from subsection 2.4, we define the "upwind" finite difference in direction $p$ as

$$
\nabla_{\mathrm{up}, \delta}^{a} v_{k, l}:=\operatorname{sgn}(a) \frac{1}{\delta}\left(v_{k, l+\operatorname{sgn}(a)}-v_{k, l}\right),
$$

and, for $\left(t, x, y, q, q_{+}, A\right) \in[0, T] \times \mathbb{R}^{5}$ and some $\theta>0$, let $S_{\delta}^{a, \text { up }}(s, t, \varphi) \in \ell^{\infty}\left(\mathcal{G}_{\delta}^{a}\right)$ be the unique solution to

$$
\begin{aligned}
S^{\mathrm{up}}\left(k, l, v_{k, l}, \nabla_{\delta}^{a} v_{k, l}, \nabla_{+, \delta}^{a} v_{k, l}, \nabla_{\mathrm{up}, \delta}^{a} v_{k, l}, \Delta_{\delta}^{a} v_{k, l}, \varphi\right) & =0, \\
v_{k, 0}=\underline{v}_{k}, v_{k, N_{\delta}^{a}} & =\bar{v}_{k},
\end{aligned}
$$

Copyright (C) by SIAM. Unauthorized reproduction of this article is prohibited. 
where

$$
S^{\text {up }}\left(k, l, v, q, q^{+}, q^{\text {up }}, A, u\right)=v-u\left(x_{k}, p_{l}\right)-h \mu q^{\text {up }}+h \widehat{F}\left(t, k \delta, v, q, q_{+}, A\right)
$$

for $k \in \mathbb{Z}, 0<l<N_{\delta}^{a},\left(v, q, q_{+}, q^{\text {up }}, A\right) \in \mathbb{R}^{5}$, and any bounded $u: \delta \mathbb{Z} \times[0,1] \rightarrow \mathbb{R}$.

The PCPT upwinding solution $v_{n, \pi, \delta}^{\text {up }}$ is then defined as in Definition 2.20 with $S_{\delta}^{a}$ replaced by $S_{\delta}^{a, \text { up }}$. By analogous steps to those in the proof of Theorem 2.21 we obtain the following.

Corollary 2.22. Under Assumptions $(H)$ and 2.10, the function $v_{n, \pi, \delta}^{\text {up }}$ converges to $v_{n}$ uniformly on compact sets as $|\pi|, \delta \rightarrow 0$, where $\pi=\left\{0=t_{0}<t_{1}<\cdots<t_{\kappa}=T\right\}$, and $\delta$ satisfy conditions (2.30), (2.31), and (2.38) for all $h=t_{j+1}-t_{j}, 0 \leq j<\kappa$.

Remark 2.23. For a linear driver, the Lax-Friedrichs flux reduces to the upwind scheme for the first derivative term in the driver and the left-hand inequality in (2.32) is also unnecessary.

2.6. Nonmonotone schemes. In this section, we take $f$ to be linear in $y, z$. As explained in the previous section, we have convergence $v_{n, \pi, \delta}^{\text {up }} \rightarrow v_{n}$ under (2.30), the left-hand inequality in (2.31), and (2.38). We can thus choose $h$ and $\delta$ such that $\frac{\delta}{h} \rightarrow 0$ as $h \rightarrow 0$.

We show that it is possible to use higher order interpolators, leading to nonmonotone schemes to approximate the unknown function $v_{n}$. This modification should lead to a practically improved convergence rate or at least efficiency improvements, although the proof we provide cannot improve the theoretical rate as we compare the nonmonotone scheme with the monotone one, following [40].

In our setting, interpolation is necessary in the $p$-variable (only) and it is therefore relevant to consider interpolators which preserve monotonicity of the dataset in the following sense, as in [24]: if the dataset $u:=\left(p_{i}, y_{i}\right)_{i=1}^{n}$ satisfies that $p_{i}<p_{j}$ implies $y_{i} \leq y_{j}$ for all $i \neq j$, then the induced function $\mathcal{I}(u)$ is nondecreasing. Although we did not prove that for all $k<\kappa$ and $l \in \mathbb{Z}$, if $v_{n, \pi, \delta}\left(t_{k+1}, x_{l}, \cdot\right)$ is nondecreasing, then $v_{n, \pi, \delta}\left(t_{k}, x_{l}, \cdot\right)$ is, it is an important feature of the quantile hedging problem and we observe it for our numerical examples in the next section. Let $n \geq 1$ be fixed and $K=\mathcal{R}_{n}^{b}$ the associated finite control set. Let $a \in K$, and let $h, \delta$ satisfy the conditions (2.30), (2.31), (2.38), and $\frac{\delta}{h} \rightarrow 0$.

Definition 2.24 (cubic spline interpolation). For any $u \in \ell^{\infty}\left(\mathcal{G}_{\delta}^{a}\right)$, we define its extension $\mathcal{I}_{\delta}^{\mathrm{CS}, a}(u): \delta \mathbb{Z} \times[0,1] \rightarrow \mathbb{R}$ by cubic spline interpolation

$$
\begin{aligned}
\mathcal{I}_{\delta}^{\mathrm{CS}, a}(u)\left(x_{k}, p\right):= & u_{k, l} h_{0,0}\left(t_{\delta}^{a}(p)\right)+\frac{1}{N_{\delta}^{a}} m_{\delta}^{a}(k, l, u) h_{1,0}\left(t_{\delta}^{a}(p)\right) \\
& +u_{k, l+1} h_{0,1}\left(t_{\delta}^{a}(p)\right)+\frac{1}{N_{\delta}^{a}} m_{\delta}^{a}(k, l+1, u) h_{1,1}\left(t_{\delta}^{a}(p)\right)
\end{aligned}
$$

for $k \in \mathbb{Z}$ and $p \in\left[p_{l}, p_{l+1}\right)$ with $0 \leq l<N_{\delta}^{a}$, and $\mathcal{I}_{\delta}^{\mathrm{CS}, a}(u)\left(x_{k}, 1\right)=u_{k, N_{\delta}^{a}}$. Here, for $(i, j) \in\{0,1\}^{2}, h_{i, j}$ are the Hermite basis functions, defined for $t \in[0,1]$ by

$$
\begin{aligned}
h_{0,0}(t) & =(1+2 t)(1-t)^{2}, & h_{1,0}(t) & =t(1-t)^{2}, \\
h_{0,1}(t) & =t^{2}(3-2 t), & h_{1,1}(t) & =t^{2}(t-1),
\end{aligned}
$$

and $t_{\delta}^{a}(p)=\frac{p-p_{l}}{p_{l+1}-p_{l}}=N_{\delta}^{a}\left(p-p_{l}\right)$ for $p \in\left[p_{l}, p_{l+1}\right)$ and $0 \leq l<N_{\delta}^{a}$, and $t_{\delta}^{a}(1)=1$. The number $m_{\delta}^{a}(k, l, u)$ is the derivative of $\mathcal{I}_{\delta}^{\mathrm{CS}, a}(u)\left(x_{k}, \cdot\right)$ at $p=p_{l}$ for each $k \in \mathbb{Z}$ and $0 \leq l \leq N_{\delta}^{a}$.

Copyright $\odot$ by SIAM. Unauthorized reproduction of this article is prohibited. 
Definition 2.25 (solution to the fully discrete nonmonotone PCPT scheme). The solution to the nonmonotone PCPT scheme associated with $\pi, \delta$ is then $v_{n, \pi, \delta}^{\mathrm{CS}}: \pi \times \delta \mathbb{Z} \times[0,1] \rightarrow \mathbb{R}$ to $\check{\mathfrak{S}}\left(\pi, \delta, j, k, p, v_{n, \pi, \delta}^{\mathrm{CS}}(t, x, p), v_{n, \pi, \delta}^{\mathrm{CS}}\right)=0$ with

$$
\check{\mathfrak{S}}(\pi, \delta, j, k, p, y, u)=\left\{\begin{aligned}
y-\min _{a \in K} \mathcal{I}_{\delta}^{\mathrm{CS}, \mathrm{a}}\left(S_{\delta}^{a}\left(t_{j}, t_{j+1}, u\left(t_{j+1}, \cdot\right)\right)\right)\left(t_{j}, x_{k}, p\right) & \text { if } j<\kappa, \\
y-g\left(x_{k}\right) p & \text { otherwise. }
\end{aligned}\right.
$$

We define, for brevity of notation below,

$$
\begin{aligned}
w_{j}^{a} & =S_{\delta}^{a}\left(t_{\kappa-j}, t_{\kappa-j+1}, v_{n, \pi, \delta}^{\mathrm{up}}\left(t_{\kappa-j+1}, \cdot\right)\right), \\
w_{j}^{\mathrm{CS}, a} & =S_{\delta}^{a}\left(t_{\kappa-j}, t_{\kappa-j+1}, v_{n, \pi, \delta}^{\mathrm{CS}}\left(t_{\kappa-j+1}, \cdot\right)\right) .
\end{aligned}
$$

Hypothesis 2.26. There exists $C \geq 0$ such that either

$$
\left[v_{n, \pi, \delta}^{\text {up }}\right]_{1}:=\sup _{(t, x, p, q) \in \pi \times \delta \mathbb{Z} \times[0,1]^{2}} \frac{\left|v_{n, \pi, \delta}^{\mathrm{up}}(t, x, p)-v_{n, \pi, \delta}^{\mathrm{up}}(t, x, q)\right|}{|p-q|} \leq C
$$

or

$$
\left|m_{\delta}^{a}\left(k, l, w_{j}^{\mathrm{CS}, a}\right)-\frac{w_{j, k, l+1}^{\mathrm{CS}, a}-w_{j, k, l}^{\mathrm{CS}, a}}{p_{l+1}-p_{l}}\right| \leq C
$$

for all $a \in K, 0 \leq j<\kappa, k \in \mathbb{Z}$, and $0 \leq l<N_{\delta}^{a}$.

Remark 2.27. A uniform Lipschitz property as in (2.39) has been shown for the standard semi-Lagrangian scheme on the whole space. This is exploited in the convergence proof in [40]. It is not clear how to obtain this for our scheme on the domain with boundaries, although the numerical tests suggest this to be true.

Theorem 2.28. Given Assumptions (H), 2.10, and 2.26 as well as (2.30), (2.31), (2.38), and $\delta / h \rightarrow 0$ as $h \rightarrow 0$, we have

$$
\left|v_{n, \pi, \delta}^{\mathrm{CS}}-v_{n, \pi, \delta}^{\mathrm{up}}\right| \rightarrow_{h \rightarrow 0} 0 .
$$

Remark 2.29. The error term $O(\delta / h)$ appearing in the proof of Theorem 2.28 is pessimistic as it only assumes an interpolation error of order 1 in $\delta$. In practice, for smooth solutions, we expect the order of the linear interpolant to be 2 and of the cubic interpolant to be 4 . In that case the requirement that $\delta / h \rightarrow 0$ can be dropped, and for a scheme which is first order in $h$ (such as the implicit Euler scheme used here) and first order in $\delta$ (due to the first order upwinding term) one may choose $h$ and $\delta$ proportional. For a higher order nonmonotone upwinding scheme such as a second order backward differentiation formula (see the good practical performance demonstrated in [6]), an even larger choice of $\delta$ would be possible, improving the efficiency further.

3. Numerical studies. We now present a numerical application of the algorithm from section 2.4.

Copyright $\odot$ by SIAM. Unauthorized reproduction of this article is prohibited. 
3.1. Model. We keep the notation of the previous section: the process $X$ is a Brownian motion with drift. In this numerical example, the drift of $Y$ is given by the following functions:

$$
\begin{aligned}
& f_{1}(t, x, y, z):=-\sigma^{-1}\left(\mu+\frac{\sigma^{2}}{2}\right) z, \text { and } \\
& f_{2}(t, x, y, z):=-\sigma^{-1}\left(\mu+\frac{\sigma^{2}}{2}\right) z+R\left(y-\sigma^{-1} z\right)^{-},
\end{aligned}
$$

where, for $x \in \mathbb{R}, x^{-}=\max (-x, 0)$ denotes the negative part of $x$. The function $f_{1}$ corresponds to a linear complete Black and Scholes market. It is well known that there are explicit formulas for the quantile hedging price for a vanilla put or call; see [23]. In both cases, we compute the quantile hedging price of a put option with strike $K=30$ and maturity $T=1$, i.e., $g(x)=\max (K-\exp (x), 0)$. The parameters of $X$ are $\sigma=0.25$ and $\mu=0.01875$ (this corresponds to $b=0.05$ for the associated geometric Brownian motion, where $\mu=b-\sigma^{2} / 2$ ).

In the rest of this section, we present the following numerical experiments. First, using the nonlinear driver $f_{2}$, we observe the convergence of $v_{\pi, \delta}$ towards $v_{n}$ for a fixed discrete control set. Second, we show that the conditions (2.30) to (2.32) are not only theoretically important, but also numerically. Last, we use the fact that the analytical solution to the quantile hedging problem with driver $f_{1}$ is known (see [23]) to assess the convergence (order) of the scheme more precisely. We observe that a judicious choice of control discretization, time and space discretization leads to convergence of $v_{\pi, \delta}$ to $v$. However, the unboundedness of the optimal control as $t \rightarrow T$ leads to expensive computations.

The scheme obtained in the previous section deals with an infinite domain in the $x$ variable. In practice, one needs to consider a bounded interval $\left[B_{1}, B_{2}\right]$ and to add some boundary conditions. Here, we choose $B_{1}=\log (10)$ and $B_{2}=\log (45)$, and the approximate Dirichlet boundary values for $v\left(t, B_{i}, p\right)$ are the limits $\lim v_{t h}(t, x, p)$ as $x \rightarrow 0$ or $x \rightarrow+\infty$, where $v_{t h}$ is the analytical solution obtained in [23] for the linear driver $f_{1}$. Since the nonlinearity in $f_{2}$ is small for realistic parameters (we choose $R=0.05$ in our tests), it is expected that the prices are close (see also [25]). Furthermore, we will consider values obtained for points $(t, x, p)$ with $x$ far from the boundary. In this situation, the influence of our choice of boundary condition should be small, as noticed, for example, in Proposition 2.17. This was studied more systematically, for example, in [2].

3.2. Convergence towards $v_{n}$ with the nonlinear driver. In this section, we consider the nonlinear driver $f_{2}$ defined above, where there is no known analytical expression for the quantile hedging price. We now fix a discrete control set, and we compute the value function $v_{\pi, \delta}$ for various discretization parameters $\pi, \delta$ satisfying (2.30) to (2.32). We consider the following control set with 22 controls:

$$
\begin{aligned}
& \left([-2,2] \cap \frac{\mathbb{Z} \backslash\{0\}}{2}\right) \cup\left([-3,3] \cap \frac{\mathbb{Z} \backslash\{0\}}{3}\right) \\
& =\{-2,-1.5, \ldots, 1.5,2\} \cup\left\{-3,-3+\frac{1}{3}, \ldots, 3-\frac{1}{3}, 3\right\},
\end{aligned}
$$

and $\delta \in\{0.1,0.07,0.05,0.03,0.01,0.007,0.005\}$. For a given $\delta$, we set $h=C \delta$ with $C:=$ $\min \left(1,2 \frac{\theta}{L}, \frac{1}{\left|\sigma^{2}-\mu\right|}\right), \theta=\frac{1}{5}$, and $L=|\mu|+R$, so that (2.30) to (2.32) are satisfied. 
Table 1

Parameters for selected values of $\delta$ for nonlinear driver test.

\begin{tabular}{|c|c|c|c|c|c|c|c|c|c|}
\hline$\delta$ & $N_{t}$ & $N_{x}$ & $N_{c}$ & $N_{p}$ & $a_{\max }$ & $N_{p}\left(a_{\max }\right)$ & $a_{\min }$ & $N_{p}\left(a_{\min }\right)$ & time $(\mathrm{sec})$ \\
\hline 0.1 & 10 & 18 & 12 & 15 & 2.5 & 2 & 0.3125 & 9 & 0.04 \\
\hline 0.05 & 20 & 33 & 15 & 38 & 2.5 & 3 & 0.3125 & 17 & 0.15 \\
\hline 0.01 & 100 & 153 & 22 & 255 & 2.77 & 10 & 0.33 & 76 & 166 \\
\hline 0.005 & 200 & 304 & 22 & 585 & 2.94 & 18 & 0.33 & 151 & 4608 \\
\hline
\end{tabular}
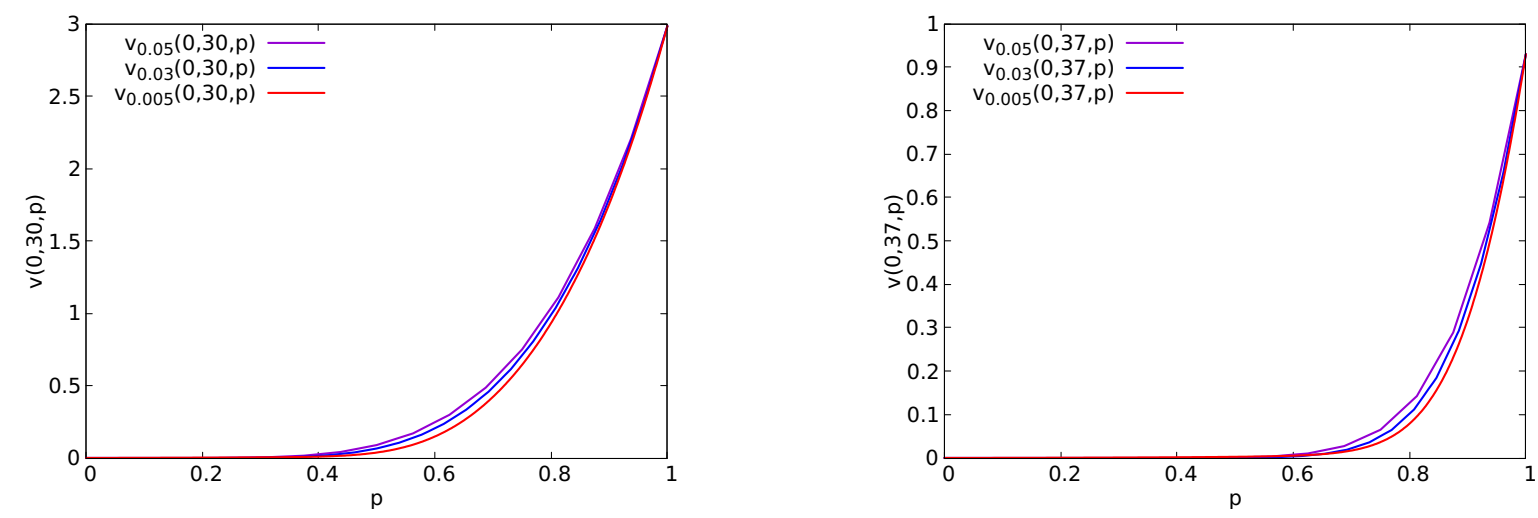

Figure 1. The functions $v_{\pi, \delta}(t, x, \cdot), t=0$ and $x \in\{30,37\}$. We drop the subscript $\pi$ above as $h=\delta$.

In Table 1, we show some discretization parameters obtained by this construction with selected values of $\delta$. Recall that different meshes are applied in each step of the PCPT algorithm for different $a$. Here, $N_{x}$ is the number of points for the $x$-variable, $N_{c}$ the number of controls, and $N_{p}$ the total number of points for the $p$ variable, i.e., for all meshes for different values of $a$ combined. Moreover, $a_{\max }$ (resp., $a_{\min }$ ) is the greatest (resp., smallest) control obtained, using the modification of the control set (3.1) as described in subsection 2.4. We also report $N_{p}\left(a_{\max }\right)$ (resp., $\left.N_{p}\left(a_{\min }\right)\right)$, the number of points for the $p$ variable for the control $a_{\max }$ (resp., $\left.a_{\min }\right)$. With our choice of parameters, we have $h=\delta$, so that the number of time steps is always $\frac{1}{\delta}$. For different choices of $\delta$, we get the graphs shown in Figure 1 for the function $p \mapsto v_{\pi, \delta}(t, x, p)$, where $(t, x)=(0,30),(0,37)$. We observe experimentally, while not proved, that the numerical approximation always gives an upper bound for $v_{n}$, which is itself greater than the quantile hedging price $v$. This, if confirmed more systematically, could be a practically useful feature of this numerical method.

The scheme preserves a key feature of the exact solution, namely, that the quantile hedging price is 0 exactly for $p$ below a certain threshold, depending on $t, x$. This is a consequence of the diffusion stencil $\Delta_{\delta}^{a}$ respecting the degeneracy of the diffusion operator $\Delta^{a}$ in $(2.20)$, which acts only in direction $(1, p)$, and by the specific construction of the meshes.

3.3. Necessity of CFL conditions. Using the same discrete control set as in section 3.2, we now fix $h=0.1$ and compute $v_{\pi, \delta}$ for $\delta$ chosen as above. The conditions (2.30) to (2.32) are then not satisfied and the results of Proposition 2.15 are not valid anymore.

First, while $\pi$ is coarse, we observe that the computational time to obtain $v_{\pi, \delta}\left(t_{j}, \cdot\right)$ from $v_{\pi, \delta}\left(t_{j+1}, \cdot\right)$ is increased. While convergence of the Picard iteration to a fixed point is still 

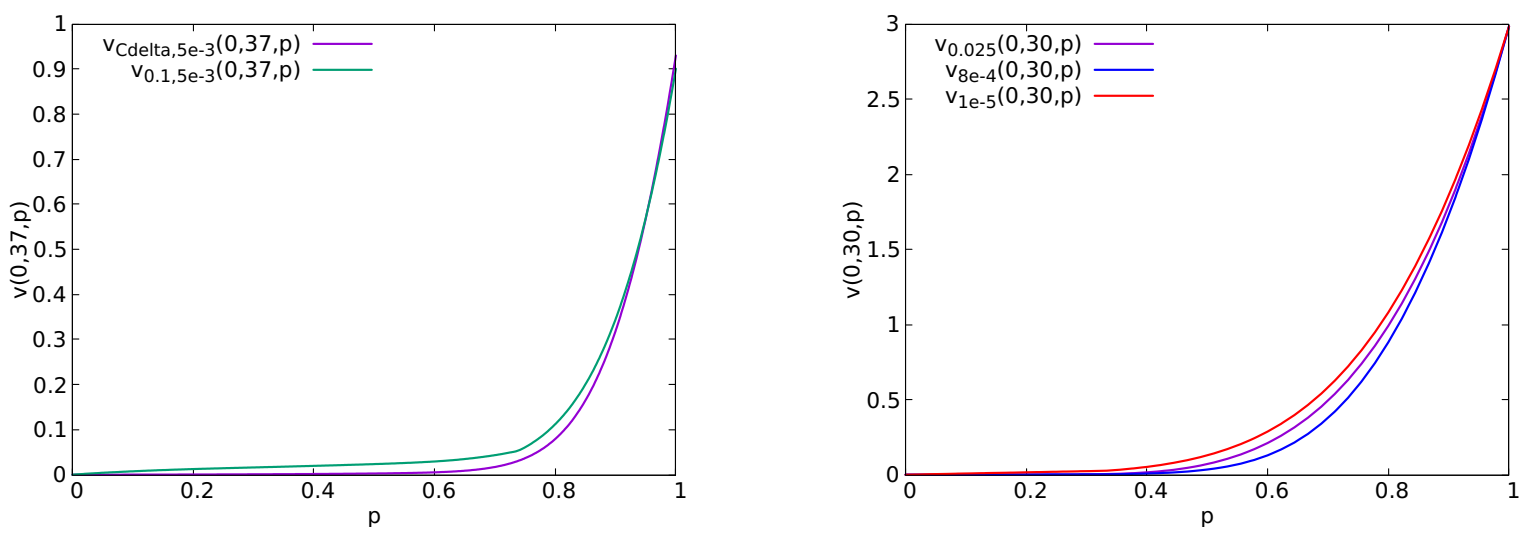

Figure 2. Left: comparison of $v_{0.1,5 e-3}(0,37, \cdot)$ and $v_{C \delta, 5 e-3}(0,37, \cdot)$. Right: comparison of $v_{h, 0.05}(0,30, \cdot)$ for different $h$ 's. We drop the subscript $\delta$ in the legend above as $\delta=0.05$ is fixed.

observed, significantly more iterations are needed: for example, for $\delta=0.005$ and $h=0.1$, 3000 Picard iterations are needed to obtain convergence subject to a tolerance of $10^{-5}$, while in the example where (2.30)-(2.32) were satisfied, 250 iterations sufficed.

The second observation is that, while we seem to observe convergence of the sequence to some limit (judging from our chosen range of $\delta$ : it might start to diverge for smaller $\delta$, as seen for the case $\delta$ fixed and varying $h$ below), it is not the limit seen in the previous subsection. We show in the left panel of Figure 2 the difference between the solution obtained with $\delta=0.005, h=0.1$, and $\delta=0.005, h=C \delta$. When conditions (2.30)-(2.32) are not met, we generally have a nonmonotone scheme, and convergence to the unique viscosity solution of the PDE, which equals the value function of the stochastic target problem, is not guaranteed.

Conversely, when $\delta$ is fixed and we vary $h$, the situation is different. There is no adverse effect on the Picard iterations, as the conditions needed for Proposition 2.15 are still satisfied. The issue here is that the consistency hypothesis is violated, and convergence to the true solution is again not observed: when $h$ is too close to 0 , the value $v_{\pi, \delta}$ grows, as seen in the right panel of Figure 2. Here, $\delta$ is fixed to 0.05 and $h$ goes from 0.025 to $1.2 \times 10^{-5}$.

3.4. Convergence to the analytical solution with linear driver. We now consider the linear driver $f_{1}$. In that case, the quantile hedging price can be found explicitly (see [23]), and, for each $(t, x, p)$, the optimal control is then

$$
\alpha(t, x, p)=\frac{1}{\sqrt{2 \pi(T-t)}} \exp \left(-\frac{N^{-1}(p)^{2}}{2}\right),
$$

where $N$ is the cumulative distribution function of the standard normal distribution.

In particular, for a fixed uniform grid $\pi=\{0, h, \ldots, \kappa h=T\}$, the optimal controls are contained in the interval $\left[0, \frac{1}{\sqrt{2 \pi h}}\right]$ on $\pi \backslash\{T\}$. On the other hand, if $\delta$ is fixed, one sees from (2.21) that the largest control one can reach (with a non-trivial $p$-grid) is $\frac{\sigma}{2 \delta}$.

For the parameters, we first choose $n \geq 2$, then we pick $\delta$ such that $\frac{\sigma}{n \delta} \geq \frac{1}{\sqrt{2 \pi C \delta}}$, and we set $h=C \delta$. It is easy to see that, therefore, $\delta$ is proportional to $n^{-2}$. 

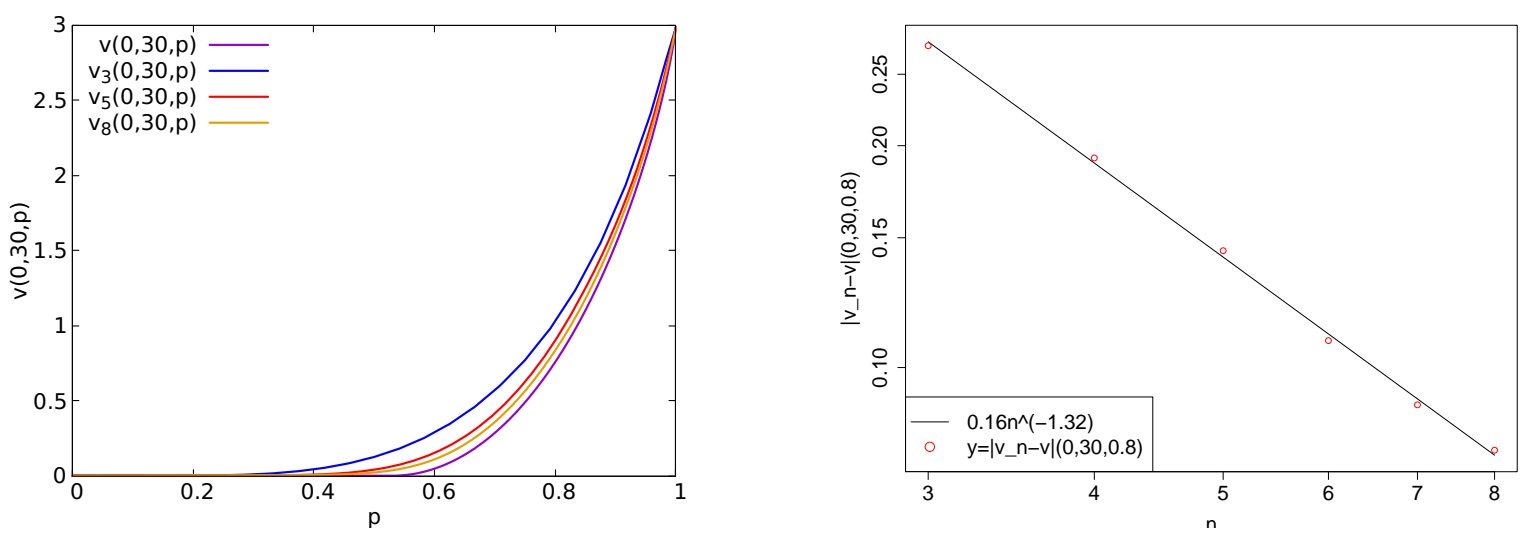

Figure 3. Left: $v_{n}(0,30, \cdot)$ and $v(0,30, \cdot)$ for $n=3,5,8$. Right: convergence rate estimation of $v_{n}(0,30,0.8)$ to $v(0,30,0.8)$ and $\log$-log plot.

Table 2

Discretization parameters for selected values of $n$ for linear driver.

\begin{tabular}{|c|c|c|c|c|c|}
\hline$n$ & $\delta$ & $a_{\max }$ & $N_{x}$ & $N_{c}$ & $N_{p}$ \\
\hline 3 & 0.04 & 1.91 & 37 & 5 & 33 \\
\hline 5 & 0.01 & 3.18 & 97 & 12 & 244 \\
\hline 7 & 0.006 & 5.09 & 248 & 26 & 1138 \\
\hline
\end{tabular}

We now pick the controls as a subset of $\left\{\frac{\sigma}{m \delta}, m \geq n\right\}$ to obtain $K_{n}:=\left\{a_{i}:=\frac{\sigma}{m_{i} \delta}, i=\right.$ $1, \ldots, M\}$ as follows: let $m_{1}=n$ so that $a_{1}=a_{\max }^{n}=\frac{\sigma}{n \delta}$. If $m_{1}, \ldots, m_{i}$ are constructed, we set $m_{i+1}=\inf \left\{m \geq m_{i}, \frac{\sigma}{m_{i} \delta}-\frac{\sigma}{m \delta} \geq \frac{1}{n}\right\}$ and $a_{i+1}=\frac{\sigma}{m_{i+1} \delta}$. If $m_{i+1}<n^{-1}$, we set $M=i+1$ and are done. This eliminates clustering of control values and speeds up the computation.

In the left panel of Figure 3, we observe convergence towards the quantile hedging price. Moreover, the right panel of Figure 3 demonstrates that the pointwise error, here for $(t, x, p)=$ $(0,30,0.8)$, has a convergence rate of about 1 with respect to $n$ in the construction described previously. Last, in Table 2, we report the values of $\delta$ and $a_{\max }$ obtained for different $n$.

3.5. Convergence using a higher order interpolation operator. We now focus on the use of a higher order interpolation operator as introduced and analyzed in section 2.6. We implement the PCPT scheme described in section 2.4, but here the linear interpolator is replaced by the monotone piecewise cubic interpolator of Fritsch and Carlson [24]. We observe, in the linear market setting, that the two solutions are indeed close; see Figure 4.

An interesting feature of the monotone piecewise cubic interpolator is that it preserves monotonicity and convexity. In particular, if the values are nondecreasing in a convex fashion, then the function obtained via the higher order interpolator is dominated by the linear interpolator. We observe this behavior in practice, even if we have not proved that $\left(v_{j, l}^{a, k}\right)_{j=0}^{N_{\delta}^{a}}$ is nondecreasing and convex.

3.6. Failure of a naive nonmonotone scheme. In this last study, we show that a simple PCPT algorithm coupled with a naive, nonmonotone finite difference scheme does not work, staying in the linear setting (as in section 3.4) for simplicity. To this end, we implement the 


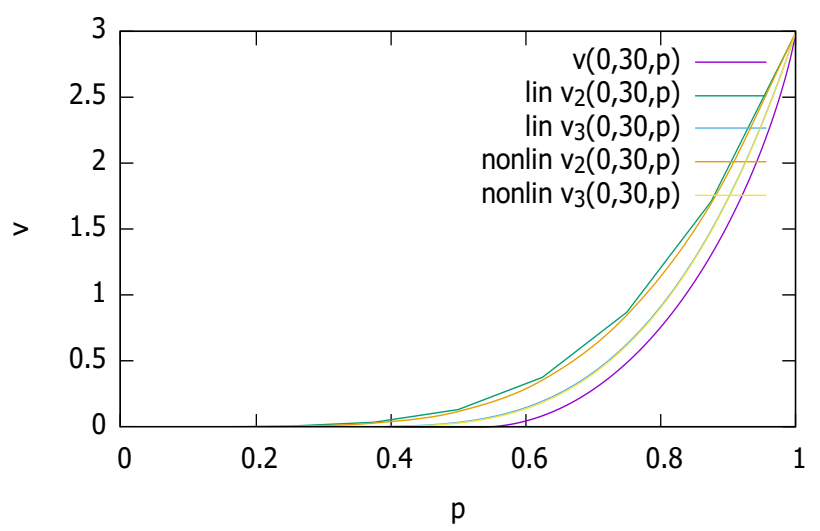

Figure 4. Comparison of the solutions to the schemes with linear and monotone cubic interpolators. The notation $v_{n}$ is as in section 3.4, specifically Figure 3.

PCPT algorithm where the PDEs in each interval $\left[t_{k}, t_{k+1}\right], 0 \leq k \leq \kappa-1$, are solved with a simple explicit finite difference scheme using the 9-point stencil for the spatial approximation (see, e.g., [27, section 13.2]).

We fix a discrete control set $K$, two reals $h, \delta>0$, a time grid $\pi=\{0<h<\cdots<\kappa h=T\}$, and a two-dimensional grid $\delta \mathbb{Z} \times\{0<\delta<\cdots<N \delta=1\}$. We then consider the PCPT scheme with a local solver $S^{\operatorname{expl}}$. Here, for $[s, t] \subset[0, T]$ and $a \in K$ and $\phi \in \delta \mathbb{Z} \times\{0, \ldots, N\} \delta$, the solution $w^{a}:=S_{\delta}^{\text {expl,a }}(s, t, \phi)$ is defined by the following relation for all $k \in \mathbb{Z}, 1 \leq l \leq N-1$ :

$$
\begin{aligned}
w_{k, l}^{a}=\phi_{k, l} & -\frac{h}{2 \delta} \frac{\sigma^{2}}{2}\left(\phi_{k+1, l}-\phi_{k-1, l}\right)-\frac{h}{2 \delta} \frac{a\left(\mu+\frac{\sigma^{2}}{2}\right)}{\sigma}\left(\phi_{k, l+1}-\phi_{k, l-1}\right) \\
& +\frac{h}{\delta^{2}} \frac{\sigma^{2}}{2}\left(\phi_{k+1, l}+\phi_{k-1, l}-2 \phi_{k, l}\right)+\frac{h}{\delta^{2}} \frac{a^{2}}{2}\left(\phi_{k, l+1}+\phi_{k, l-1}-2 \phi_{k, l}\right) \\
& +\frac{h}{4 \delta^{2}} a \sigma\left(\phi_{k+1, l+1}+\phi_{k-1, l-1}-\phi_{k+1, l-1}-\phi_{k-1, l+1}\right)
\end{aligned}
$$

with the usual boundary conditions. We denote by $v_{K}^{\operatorname{expl}}$ the solution of the PCPT scheme.

First, this operator is not monotone in the sense of (2.33) for any $a \neq 0, h, \delta>0$, due to the mixed signs of the last term. Hence, a comparison theorem is not available. In particular, we cannot theoretically guarantee the positivity of the solution, nor can we ensure the stability.

In the tests, we choose $K_{2}=\{-2,-1.5,-1,0.5,0,0.5,1,1.5,2\}$ and $K_{3}=K_{2} \cup$ $\left\{\frac{k}{3}, k \in\{-9,9\}\right\}$. The model parameters are as previously. We plot in Figures 5 and 6 the solution to the scheme with $\delta=0.1$ and $h=\frac{\delta^{2}}{\max _{a \in K}|a|^{2}}$ in the cases $x=30$ and $x=37$.

We observe that indeed the solution we obtain becomes negative and is not nondecreasing in its $p$ argument. So it is clearly not satisfactory with respect to these key properties of the quantile hedging price. Moreover, we observe that this undesired behavior is more pronounced when we take $K_{3}$ instead of $K_{2}$.

Copyright (C) by SIAM. Unauthorized reproduction of this article is prohibited. 

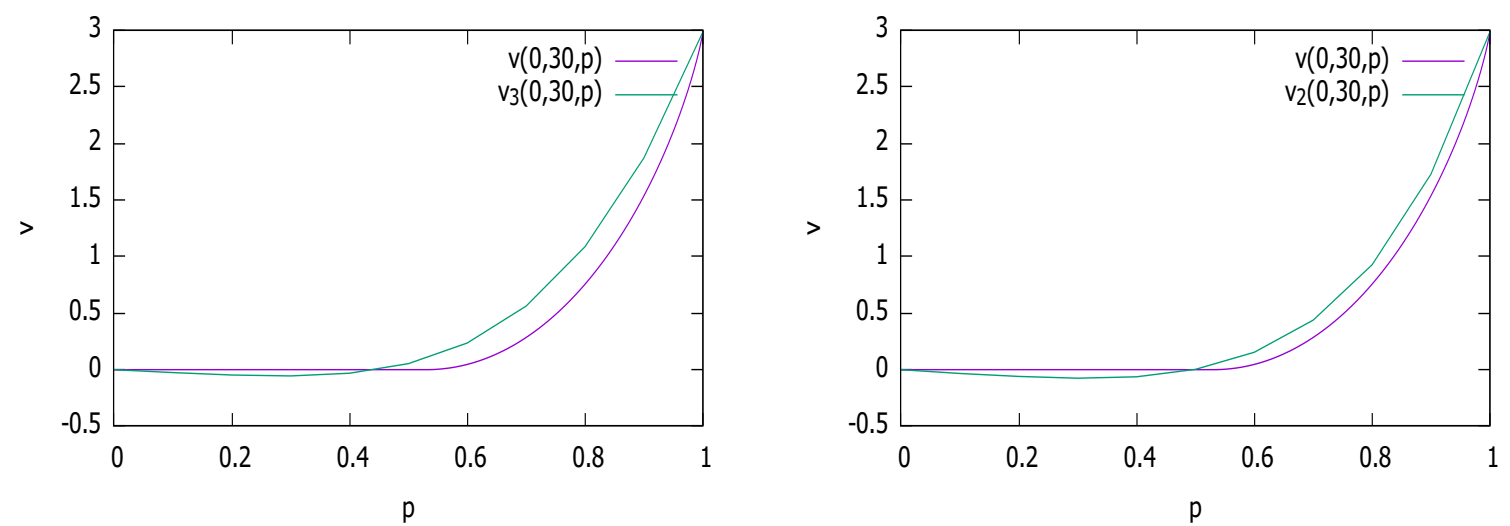

Figure 5. Naive finite difference scheme. Left: $v_{K_{2}}^{\text {expl }}(0,30, \cdot)$. Right: $v_{K_{3}}^{\operatorname{expl}}(0,30, \cdot)$.
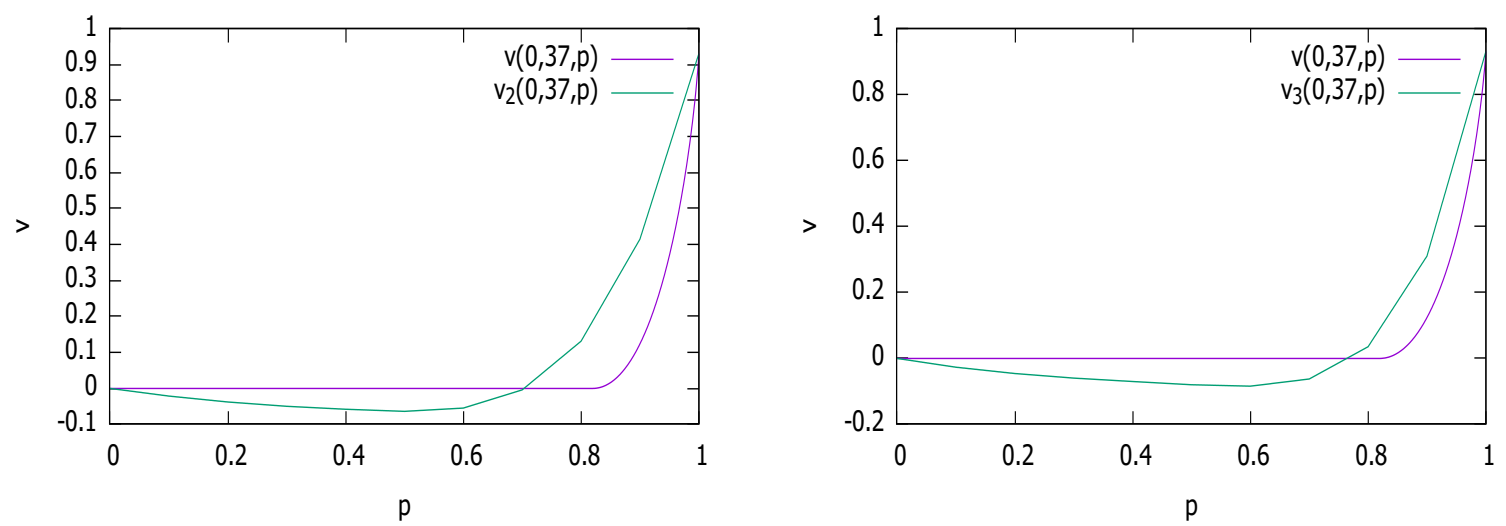

Figure 6. Naive finite difference scheme. Left: $v_{K_{2}}^{\operatorname{expl}}(0,37, \cdot)$. Right: $v_{K_{3}}^{\operatorname{expl}}(0,37, \cdot)$.

\section{Proofs of results in section 2.}

4.1. Proof of Proposition 2.3. We first recall the comparison theorem for the PDEs of the form (2.7); see, e.g., [14].

Proposition 4.1. Let $A \subset \mathbb{R}^{d}$ be a finite set, $0 \leq \tau<\theta \leq T$, and $u_{1}$ (resp., $u_{2}$ ) be a lower semicontinuous supersolution (resp., upper semicontinuous subsolution) with polynomial growth, of

$$
-\partial_{t} \varphi+\mathcal{F}_{A}\left(t, x, \varphi, D \varphi, D^{2} \varphi\right)=0 \text { on }[\tau, \theta) \times \mathbb{R}^{d} \times(0,1)
$$

with $\mathcal{F}_{A}(\Xi)=\sup _{a \in A} F^{a}(\Xi)$ for $\Xi:=(t, x, y, q, A)$ as in the beginning of subsection 2.3. Assume that $u_{1} \geq u_{2}$ on $\left([\tau, \theta] \times \mathbb{R}^{d} \times\{0,1\}\right) \bigcup\left(\{\theta\} \times \mathbb{R}^{d} \times[0,1]\right)$. Then $u_{1} \geq u_{2}$ on $[\tau, \theta] \times$ $\mathbb{R}^{d} \times[0,1]$. 
Corollary 4.2. Let $\mathcal{R} \subset \mathcal{S} \backslash \mathcal{D}$ be a finite set. There exists a unique continuous solution $w$ to (4.1) or, equivalently,

$$
\sup _{\eta \in \mathcal{R}} H^{\eta}\left(t, x, \varphi, \partial_{t} \varphi, D \varphi, D^{2} \varphi\right)=0 \text { on }[\tau, \theta) \times \mathbb{R}^{d} \times(0,1),
$$

satisfying $w(\cdot)=\Psi(\cdot)$ on $\left([\tau, \theta] \times \mathbb{R}^{d} \times\{0,1\}\right) \bigcup\left(\{\theta\} \times \mathbb{R}^{d} \times[0,1]\right)$, where $\Psi \in \mathcal{C}^{0}$.

Proof. This is a direct application of the comparison principles. The equivalence between (4.1) with $A:=\mathcal{R}^{b}$ and (4.2) comes from the fact that $H^{\eta}(\Theta)$ and $-b-F^{\eta^{b}}(\Xi)$ have the same sign, where $\Xi=(t, x, y, q, A)$ and $\Theta=(t, x, y, b, q, A)$.

We are now in a position to complete the proof of Proposition 2.3.

1. For $n^{\prime}<n$, we observe that $v_{n^{\prime}}$ is a supersolution of (2.7) as $\mathcal{R}_{n^{\prime}} \subset \mathcal{R}_{n}$. Using the comparison result of Proposition 4.1, we obtain that $v_{n^{\prime}} \geq v_{n}$. Similarly, using the comparison principle [12, Theorem 3.2], we obtain that $v_{n} \geq v$, for all $n \geq 1$.

For all $(t, x, p) \in[0, T] \times(0, \infty)^{d} \times[0,1]$, let

$$
\begin{aligned}
& \bar{v}(t, x, p)=\lim _{j \rightarrow \infty} \sup \left\{v_{n}(s, y, q): n \geq j \text { and }\|(s, y, q)-(t, x, p)\| \leq \frac{1}{j}\right\}, \\
& \underline{v}(t, x, p)=\lim _{j \rightarrow \infty} \inf \left\{v_{n}(s, y, q): n \geq j \text { and }\|(s, y, q)-(t, x, p)\| \leq \frac{1}{j}\right\} .
\end{aligned}
$$

From the above discussion, recalling that $v_{1}$ and $v$ are continuous, we have

$$
v_{1} \geq \bar{v} \geq \underline{v} \geq v,
$$

which shows that $\bar{v}$ and $\underline{v}$ satisfy the boundary conditions (2.4)-(2.5).

In order to prove the theorem, it is enough to show that $\bar{v}$ is a viscosity subsolution of (2.3) and $\underline{v}$ is a viscosity supersolution (which follows similarly and is therefore omitted). The comparison principle [12, Theorem 3.2] then implies that $v=\bar{v}=\underline{v}$, and it follows from [14, Remark 6.4] that the convergence $v_{n} \rightarrow v$ as $n \rightarrow \infty$ is uniform on every compact set. Using Theorem 6.2 in [1], we obtain that $\bar{v}$ is a subsolution to

$$
\underline{\mathcal{H}}\left(t, x, \varphi, \partial_{t} \varphi, D \varphi, D^{2} \varphi\right)=0 \text { on }[0, T) \times(0, \infty)^{d} \times(0,1),
$$

where

$$
\underline{\mathcal{H}}(\Theta)=\lim _{j \rightarrow \infty} \inf \left\{\mathcal{H}_{n}\left(\Theta^{\prime}\right): n \geq j \text { and }\left\|\Theta-\Theta^{\prime}\right\| \leq \frac{1}{j}\right\} .
$$

In the next step, we prove that $\underline{\mathcal{H}}=\mathcal{H}$, which concludes the proof of the proposition.

2. Let us denote by $\mathfrak{P}_{n}: \mathcal{S} \rightarrow \tilde{\mathcal{S}}_{n}$ the closest neighbor projection on the closed set $\tilde{\mathcal{S}}_{n}$. From (2.6), we have that $\lim _{n \rightarrow \infty} \mathfrak{P}_{n}(\eta)=\eta$ for all $\eta \in \mathcal{S}$. We also have that

$$
\mathcal{H}(\Theta)=H^{\eta^{*}}(\Theta)
$$

for some $\eta^{*} \in \operatorname{argmax}_{\eta \in \mathcal{S}} H^{\eta}(\Theta)$ as $\mathcal{S}$ is compact. Let us now introduce $\eta_{n}:=\mathfrak{P}_{n}\left(\eta^{*}\right)$ and by continuity of $H$, we have

$$
H^{\eta_{n}}(\Theta) \rightarrow \mathcal{H}(\Theta) \text { as } n \rightarrow \infty
$$


We also observe that

$$
H^{\eta_{n}}(\Theta) \leq \mathcal{H}_{n}(\Theta) \leq \mathcal{H}(\Theta)
$$

This proves the convergence $\mathcal{H}_{n}(\Theta) \uparrow \mathcal{H}(\Theta)$, for all $\Theta$. As $\mathcal{H}$ is continuous, we conclude by using Dini's theorem that the convergence is uniform on compact subsets, leading to $\underline{\mathcal{H}}=\mathcal{H}$.

4.2. Proof of Theorem 2.7. We first check the consistency with the boundary conditions. Let $\hat{a} \in K$ and $\hat{w}$ be the (continuous) solution of

$$
-\partial_{t} \varphi+F^{\hat{a}}\left(t, x, \varphi, D \varphi, D^{2} \varphi\right)=0 \text { on }[0, T) \times \mathbb{R}^{d} \times(0,1)
$$

with boundary condition $v(t, x, p)=p V(t, x)$ on $[0, T] \times \mathbb{R}^{d} \times\{0,1\} \bigcup\{T\} \times \mathbb{R}^{d} \times[0,1]$. By backward induction on $\pi$, one gets that

$$
v_{n, \pi} \leq \hat{w} .
$$

Indeed, we have $v_{n, \pi}(T, \cdot)=\hat{w}(T, \cdot)$. Now if the inequality is true at time $t_{k}, k \geq 1$, we have, using the comparison result for (4.3), recalling Proposition 4.1, that

$$
w^{k, \hat{a}}(t, \cdot) \leq \hat{w}(t, \cdot) \text { for } t \in\left[t_{k-1}, t_{k}\right],
$$

and thus a fortiori $\hat{w}(t, \cdot) \geq v_{n, \pi}(t, \cdot)$ for $t \in\left[t_{k-1}, t_{k}\right]$.

We also obtain that $v_{n, \pi} \geq v_{n}$ by backward induction. Indeed, we have $v_{n, \pi}(T, \cdot)=v_{n}(T, \cdot)$. Assume that the inequality is true at time $t_{k}, k \geq 1$. We observe that $w^{k, a}$ is a supersolution of (2.7), namely, the PDE satisfied by $v_{n}$. By the comparison result, we have $w^{k, a}(t, \cdot) \geq v_{n}(t, \cdot)$ for $t \in\left[t_{k-1}, t_{k}\right]$. Taking the infimum over $a \in K$ then yields the inequality.

Since $v_{n} \leq \underline{w} \leq \bar{w} \leq \hat{w}$, where

$$
\bar{w}(t, x, p)=\limsup _{\left(t^{\prime}, x^{\prime}, p^{\prime},|\pi|\right) \rightarrow(t, x, p, 0)} v_{n, \pi}\left(t^{\prime}, x^{\prime}, p^{\prime}\right) \text { and } \underline{w}=\liminf _{\left(t^{\prime}, x^{\prime}, p^{\prime},|\pi|\right) \rightarrow(t, x, p, 0)} v_{n, \pi}\left(t^{\prime}, x^{\prime}, p^{\prime}\right),
$$

we obtain that $\underline{w}$ and $\bar{w}$ satisfy the boundary conditions (2.4)-(2.5).

We now prove the monotonicity (see Proposition 4.3), stability (see Proposition 4.6), and consistency in the interior of the domain (see Proposition 4.9) for the discrete-time PCPT scheme, by a combination of viscosity solution arguments and-mostly-BSDE arguments where they appear more natural. It should be possible to use only PDE arguments with similar main steps as in [3]. Combining this with the consistency with the boundary conditions above and [4, Theorem 2.1] then ensures the convergence in $\mathcal{C}^{0}$ of $v_{n, \pi}$ to $v_{n}$ as $|\pi| \rightarrow 0$.

Proposition 4.3 (monotonicity). For $u \geq v \in \mathcal{B C},(t, x, p) \in[0, T] \times \mathbb{R}^{d} \times[0,1]$, and $y \in \mathbb{R}$, we have $\mathfrak{S}(\pi, t, x, p, y, u) \leq \mathfrak{S}(\pi, t, x, p, y, v)$.

Proof. Let $t<T, x \in \mathbb{R}^{d}, p \in[0,1]$. By the definition of $v_{n, \pi}$, recalling (2.16), it is sufficient to prove that, for any $a \in K$, we have $S^{a}\left(t^{-}, t^{+}, u\left(t^{+}, \cdot\right)\right)(t, x, p) \geq S^{a}\left(t^{-}, t^{+}, v\left(t^{+}, \cdot\right)\right)(t, x, p)$, with $t^{-}, t^{+}$defined in (2.13). But this follows directly from Proposition 4.1.

We now study the stability of the scheme. We first show that the solution of the scheme $v_{n, \pi}$ is increasing in its third variable. This is not only an interesting property in its own right 
which the piecewise constant policy solution inherits from the solution to the original problem (2.2), but it also allows us to obtain easily a uniform bound for $v_{n, \pi}$, namely, the boundary condition at $p=1$. We provide first a probabilistic representation for $w^{j, a}(0 \leq j<\kappa, a \in K)$ as solutions to BSDEs with terminal time $t_{j+1}$.

Lemma 4.4. (i) Let $a \in \mathbb{R}^{d}$ and $w^{a}$ be the unique solution to

$$
-\partial_{t} \varphi+F^{a}\left(t, x, \varphi, D \varphi, D^{2} \varphi\right)=0 \text { on }[\tau, \theta) \times \mathbb{R}^{d} \times(0,1),
$$

satisfying $w^{a}(\cdot)=\Psi(\cdot)$ on $[\tau, \theta] \times \mathbb{R}^{d} \times\{0,1\} \bigcup\{\theta\} \times \mathbb{R}^{d} \times[0,1]$, where $\Psi \in \mathcal{C}^{0}$. Then it admits the probabilistic representation $w^{a}(t, x, p)=Y_{t}$, where $Y$ is the first component of the solution $(Y, Z)$ to the following BSDE with random terminal time:

$$
Y .=\Psi\left(\mathcal{T}, X_{\mathcal{T}}^{t, x}, P_{\mathcal{T}}^{t, p, a}\right)+\int_{.}^{\mathcal{T}} f\left(s, X_{s}^{t, x}, Y_{s}, Z_{s}\right) \mathrm{d} s-\int_{.}^{\mathcal{T}} Z_{s} \mathrm{~d} W_{s}
$$

with $\mathcal{T}:=\inf \left\{s \geq t: P_{s}^{t, p, a} \in\{0,1\}\right\} \wedge \theta$ and $P^{t, p, a}:=p+a\left(W .-W_{t}\right)$.

(ii) Assume moreover, that $\Psi(T, \cdot)=\phi(\cdot)$ and $\Psi(\cdot, 1)=B^{1}(\cdot, \phi), \Psi(\cdot, 0)=B^{0}(\cdot, \phi)$ with the notation of (2.12). Then the solution $(\tilde{Y}, \tilde{Z})$ to

$$
Y .=\phi\left(X_{\theta}^{t, x}, \tilde{P}_{\theta}^{t, p, a}\right)+\int_{.}^{\theta} f\left(s, X_{s}^{t, x}, Y_{s}, Z_{s}\right) \mathrm{d} s-\int_{.}^{\theta} Z_{s} \mathrm{~d} W_{s},
$$

where $\tilde{P}^{t, p, a}:=P_{. \wedge \mathcal{T}}^{t, p, a}$, satisfies $Y=\tilde{Y}$ on $[t, \mathcal{T}]$.

Proof. (i) The probabilistic representation is proved in [16]. Note that uniqueness to the PDE comes from Corollary 4.2 in the special case where $\mathcal{R}$ is a singleton.

(ii) Let $A:=\{\mathcal{T}=\theta\}, B:=\left\{\mathcal{T}<\theta, P_{\mathcal{T}}^{p, a}=1\right\}$, and $C:=\left\{\mathcal{T}<\theta, P_{\mathcal{T}}^{p, a}=0\right\}$, so that $\Omega=A \cup B \cup C$. For $e \in\{0,1\}$, let $\left({ }^{e} Y^{t, x},{ }^{e} Z^{t, x}\right)$ be the solution to

$$
Y .=\phi\left(X_{\theta}^{t, x}, e\right)+\int_{.}^{\theta} f\left(s, X_{s}^{t, x}, Y_{s}, Z_{s}\right) \mathrm{d} s-\int_{.}^{\theta} Z_{s} \mathrm{~d} W_{s} .
$$

By (2.12), we have $B^{e}\left(\tau, X_{\tau}^{t, x}, \phi\right)={ }^{e} Y_{\tau}$ for $e \in\{0,1\}$.

We introduce the following auxiliary processes for $s \in[t, \theta]$ :

$$
\begin{aligned}
& \check{Y}_{s}:=Y_{s} 1_{t \leq s \leq \mathcal{T}}+{ }^{1} Y_{s} 1_{s>\mathcal{T}} 1_{B}+{ }^{0} Y_{s} 1_{s>\mathcal{T}} 1_{C}, \\
& \check{Z}_{s}:=Z_{t} 1_{t \leq s \leq \mathcal{T}}+{ }^{1} Z_{t} 1_{s>\mathcal{T}} 1_{B}+{ }^{0} Z_{t} 1_{s>\mathcal{T}} 1_{C} .
\end{aligned}
$$

First, by construction, we have $Y=\check{Y}$ on $[t, \mathcal{T}]$. To prove the proposition it is thus sufficient to show that $\tilde{Y}=\check{Y}$ on $[t, \theta]$. To this effect, we show that $(\check{Y}, \check{Z})$ is solution of (4.4). We have, for all $s \in[t, \theta]$,

$$
\begin{aligned}
\check{Y}_{s}= & {\left[\Psi\left(\mathcal{T}, X_{\mathcal{T}}^{t, x}, P_{\mathcal{T}}^{t, p, a}\right)+\int_{s}^{\mathcal{T}} f\left(u, X_{u}^{t, x}, Y_{u}, Z_{u}\right) \mathrm{d} u-\int_{s}^{\mathcal{T}} Z_{u} \mathrm{~d} W_{u}\right] 1_{s \leq \mathcal{T}} } \\
& +\left[\phi\left(X_{\theta}^{t, x}, 1\right)+\int_{s}^{\theta} f\left(u, X_{u}^{t, x},{ }^{1} Y_{u},{ }^{1} Z_{u}\right) \mathrm{d} u-\int_{s}^{\theta}{ }^{1} Z_{u} \mathrm{~d} W_{u}\right] 1_{\mathcal{T}<s} 1_{B} \\
& +\left[\phi\left(X_{\theta}^{t, x}, 0\right)+\int_{s}^{\theta} f\left(u, X_{u}^{t, x},{ }^{0} Y_{u},{ }^{0} Z_{u}\right) \mathrm{d} u-\int_{s}^{\theta}{ }^{0} Z_{u} \mathrm{~d} W_{u}\right] 1_{\mathcal{T}<s} 1_{C} .
\end{aligned}
$$

Copyright (C) by SIAM. Unauthorized reproduction of this article is prohibited. 
By our hypotheses and the definition of ${ }^{p} Y$, since $\tilde{P}_{\theta}^{t, p, a}=1$ on $B$ and $\tilde{P}_{\theta}^{t, p, a}=0$ on $C$,

$$
\begin{aligned}
\Psi\left(\mathcal{T}, X_{\mathcal{T}}^{t, x}, P_{\mathcal{T}}^{t, p, a}\right)= & \phi\left(X_{\theta}^{t, x}, P_{\theta}^{t, p, a}\right) 1_{A}+B^{1}\left(\mathcal{T}, X_{\mathcal{T}}^{t, x}, \phi\right) 1_{B}+B^{0}\left(\mathcal{T}, X_{\mathcal{T}}^{t, x}, \phi\right) 1_{C} \\
= & \phi\left(X_{\theta}^{t, x}, P_{\theta}^{t, p, a}\right) 1_{A}+{ }^{1} Y_{\mathcal{T}} 1_{B}+{ }^{0} Y_{\mathcal{T}} 1_{C} \\
= & \phi\left(X_{\theta}^{t, x}, \tilde{P}_{\theta}^{t, p, a}\right) \\
& +\left[\int_{\mathcal{T}}^{\theta} f\left(u, X_{u}^{t, x},{ }^{1} Y_{u},{ }^{1} Z_{u}\right) \mathrm{d} u-\int_{\mathcal{T}}^{\theta}{ }^{1} Z_{u} \mathrm{~d} W_{u}\right] 1_{B} \\
& +\left[\int_{\mathcal{T}}^{\theta} f\left(u, X_{u}^{t, x},{ }^{0} Y_{u},{ }^{0} Z_{u}\right) \mathrm{d} u-\int_{\mathcal{T}}^{\theta}{ }^{0} Z_{u} \mathrm{~d} W_{u}\right] 1_{C} .
\end{aligned}
$$

Thus, since $A \cap\{\mathcal{T}<s\}=\emptyset$, we deduce

$$
\begin{aligned}
\check{Y}_{s}= & \phi\left(X_{\theta}^{t, x}, \tilde{P}_{\theta}^{t, p, a}\right) \\
+ & {\left[\int_{\mathcal{T}}^{\theta} f\left(u, X_{u}^{t, x},{ }^{1} Y_{u},{ }^{1} Z_{u}\right) \mathrm{d} u 1_{s \leq \mathcal{T}} 1_{B}+\int_{\mathcal{T}}^{\theta} f\left(u, X_{u}^{t, x},{ }^{0} Y_{u},{ }^{0} Z_{u}\right) \mathrm{d} u 1_{s \leq \mathcal{T}} 1_{C}\right] } \\
+ & {\left[\int_{s}^{\mathcal{T}} f\left(u, X_{u}^{t, x}, Y_{u}, Z_{u}\right) \mathrm{d} u 1_{s \leq \tau}+\int_{s}^{\mathcal{T}} f\left(u, X_{u}^{t, x},{ }^{1} Y_{u},{ }^{1} Z_{u}\right) \mathrm{d} u 1_{\mathcal{T}<s} 1_{B}\right.} \\
& \left.+\int_{s}^{\mathcal{T}} f\left(u, X_{u}^{t, x},{ }^{0} Y_{u},{ }^{0} Z_{u}\right) \mathrm{d} u 1_{\mathcal{T}<s} 1_{C}\right] \\
& -\left[\int_{\mathcal{T}}{ }^{1} Z_{u} \mathrm{~d} W_{u} 1_{s \leq \mathcal{T}} 1_{B}+\int_{\mathcal{T}}{ }^{0} Z_{u} \mathrm{~d} W_{u} 1_{s \leq \mathcal{T}} 1_{C}\right] \\
& -\left[\int_{s}^{\mathcal{T}} Z_{u} \mathrm{~d} W_{u} 1_{s \leq \tau}+\int_{s}^{\mathcal{T}}{ }^{1} Z_{u} \mathrm{~d} W_{u} 1_{\mathcal{T}<s} 1_{B}+\int_{s}{ }^{0} Z_{u} \mathrm{~d} W_{u} 1_{\mathcal{T}<s} 1_{C}\right] .
\end{aligned}
$$

Now, since $\left({ }^{1} Y_{u},{ }^{1} Z_{u}\right)=\left(\check{Y}_{u}, \check{Z}_{u}\right)$ on $(\mathcal{T}, \theta] \cap B$ and $\left({ }^{0} Y_{u},{ }^{0} Z_{u}\right)=\left(\check{Y}_{u}, \check{Z}_{u}\right)$ on $(\mathcal{T}, \theta] \cap B$, and $\int_{\mathcal{T}}^{\theta} f\left(u, X_{u}^{t, x}, \check{Y}_{u}, \check{Z}_{u}\right) \mathrm{d} u 1_{A}=0$, we get

$$
\begin{gathered}
\int_{\mathcal{T}}^{\theta} f\left(u, X_{u}^{t, x},{ }^{1} Y_{u},{ }^{1} Z_{u}\right) \mathrm{d} u 1_{s \leq \mathcal{T}} 1_{B}+\int_{\mathcal{T}}^{\theta} f\left(u, X_{u}^{t, x},{ }^{0} Y_{u},{ }^{0} Z_{u}\right) \mathrm{d} u 1_{s \leq \mathcal{T}} 1_{C} \\
=\int_{\mathcal{T}}^{\theta} f\left(u, X_{u}^{t, x}, \check{Y}_{u}, \check{Z}_{u}\right) \mathrm{d} u 1_{s \leq \mathcal{T}} .
\end{gathered}
$$

A similar analysis for the other terms shows that $(\check{Y}, \check{Z})$ is a solution to $(4.4)$ and concludes the proof.

Lemma 4.5. For all $t \in[0, T], x \in \mathbb{R}^{d}, 0 \leq q \leq p \leq 1$, we have $v_{n, \pi}(t, x, q) \leq v_{n, \pi}(t, x, p)$.

Proof. We will prove the assertion by induction on $j \in\{0, \ldots, \kappa\}$. For $t=T=t_{\kappa}$ and every $x \in \mathbb{R}^{d}$, we have $(x, p) \mapsto v_{n, \pi}(T, x, p):=g(x) p$, which is increasing in $p$.

Let $1 \leq j<\kappa-1$. Assume now that $v_{n, \pi}(t, x, \cdot)$ is increasing for all $t \geq t_{j+1}$ and $x \in \mathbb{R}^{d}$. We show that $v_{n, \pi}(t, x, \cdot)$ is also increasing for $t \in\left[t_{j}, t_{j+1}\right)$ and $x \in \mathbb{R}^{d}$.

Let $0 \leq q \leq p \leq 1$. By the definition of $v_{n, \pi}$ in (2.16), it is sufficient to show that for each $a \in K$, we have, for $(t, x) \in[0, T] \times \mathbb{R}^{d}, w^{j, a}(t, x, q) \leq w^{j, a}(t, x, p)$. From Lemma 4.4(i), these

Copyright (C) by SIAM. Unauthorized reproduction of this article is prohibited. 
two quantities admit a probabilistic representation with two different random terminal times

$$
\begin{aligned}
& \tau^{q}=\inf \left\{s \geq t: P_{s}^{t, q, a} \in\{0,1\}\right\} \wedge t_{k+1} \\
& \tau^{p}=\inf \left\{s \geq t: P_{s}^{t, p, a} \in\{0,1\}\right\} \wedge t_{k+1} .
\end{aligned}
$$

However, using Lemma 4.4(ii), we can write probabilistic representations with BSDEs with terminal time $t_{j+1}$ : we have that $S^{a}\left(t_{j}, t_{j+1}, w_{\pi}\left(t_{j+1}, \cdot\right)\right)(t, x, p)=\tilde{Y}_{t}^{t, x, p, a}$, where $\tilde{Y}_{t}^{t, x, p, a}$ is the first component of the solution of the following BSDE:

$$
Y_{s}=v_{n, \pi}\left(t_{j+1}, X_{t_{j+1}}^{t, x}, \tilde{P}_{t_{j+1}}^{t, p, a}\right)+\int_{s}^{t_{j+1}} f\left(u, X_{u}^{t, x}, Y_{u}, Z_{u}\right) \mathrm{d} u-\int_{s}^{t_{j+1}} Z_{u} \mathrm{~d} W_{u},
$$

where $\tilde{P}^{t, p, a}$ is the process defined by:

$$
\tilde{P}_{s}^{t, p, a}=p+\int_{t}^{s} a 1_{\left\{u \leq \tau^{p}\right\}} \mathrm{d} W_{u}
$$

and a similar representation holds for $S^{a}\left(t_{j}, t_{j+1}, w_{\pi}\left(t_{j+1}, \cdot\right)\right)(t, x, q)$.

It remains to show that

$$
v_{n, \pi}\left(t_{j+1}, X_{t_{j+1}}^{t, x}, \tilde{P}_{t_{j+1}}^{t, p, a}\right) \geq v_{n, \pi}\left(t_{j+1}, X_{t_{j+1}}^{t, x}, \tilde{P}_{t_{j+1}}^{t, q, a}\right) .
$$

If this is true, the classical comparison theorem for BSDEs (see, e.g., [21, Theorem 2.2]), concludes the proof.

First, we observe that $P_{\tau_{p}}^{t, p, a} \geq P_{\tau_{p}}^{t, q, a}$. On $\left\{\tau_{p}=T\right\}$, (4.5) holds straightforwardly by the induction hypothesis. On $\left\{\tau_{p}<T\right\}$, if $P_{\tau_{p}}^{t, p, a}=1$, then $P_{T}^{t, p, a}=1$ and (4.5) holds by the induction hypothesis, as $P_{T}^{t, q, a} \leq 1$; if $P_{\tau_{p}}^{t, p, a}=0$, then a fortiori $P_{\tau_{p}}^{t, q, a}=0$ and $P_{T}^{t, p, a}=P_{T}^{t, q, a}=0$, which concludes the proof.

Proposition 4.6 (stability). The solution to (2.15) is bounded independently of $\pi$.

Proof. For any time grid $\pi$ and any $(t, x, p) \in[0, T] \times \mathbb{R}^{d} \times[0,1]$, we have $0=v_{n, \pi}(t, x, 0) \leq$ $v_{n, \pi}(t, x, p) \leq v_{n, \pi}(t, x, 1)=V(t, x)$.

To prove the consistency of the scheme, we will need the two following lemmas.

Lemma 4.7. For $0 \leq \tau \leq t \leq \theta \leq T, \xi \in \mathbb{R}$, and $\phi \in \mathcal{C}^{\infty}\left([0, T] \times \mathbb{R}^{d} \times[0,1]\right)$, it holds

$$
\left|S^{a}(\tau, \theta, \phi(\theta, \cdot)+\xi)(t, \cdot)-S^{a}(\tau, \theta, \phi(\theta, \cdot))(t, \cdot)-\xi\right|_{\infty} \leq C|\theta-t||\xi| .
$$

Proof. We denote $w=S^{a}(\tau, \theta, \phi(\cdot))$ and $\tilde{w}=S^{a}(\tau, \theta, \phi(\cdot)+\xi)$. Using Lemma 4.4, we have that, for $(t, x, p) \in[\tau, \theta] \times \mathbb{R}^{d} \times[0,1]$,

$$
w(t, x, p)=Y_{t} \text { and } \tilde{w}(t, x, p)=\hat{Y}_{t},
$$

where $(Y, Z)$ and $(\hat{Y}, \hat{Z})$ are solutions to, respectively,

$$
\begin{aligned}
& Y_{r}=\phi\left(X_{\theta}^{t, x}, \tilde{P}_{\theta}^{t, p, a}\right)+\int_{r}^{T} f\left(s, X_{s}^{t, x}, Y_{s}, Z_{s}\right) \mathrm{d} s-\int_{r}^{\theta} Z_{s} \mathrm{~d} W_{s}, t \leq r \leq \theta, \\
& \hat{Y}_{r}=\phi\left(X_{\theta}^{t, x}, \tilde{P}_{\theta}^{t, p, a}\right)+\xi+\int_{r}^{T} f\left(s, X_{s}^{t, x}, \hat{Y}_{s}, \hat{Z}_{s}\right) \mathrm{d} s-\int_{r}^{\theta} \hat{Z}_{s} \mathrm{~d} W_{s}, t \leq r \leq \theta .
\end{aligned}
$$

Copyright (C by SIAM. Unauthorized reproduction of this article is prohibited. 
Denoting $\Gamma:=Y+\xi$ and $f_{\xi}(t, x, y, z):=f(t, x, y-\xi, z)$, we observe that $(\Gamma, Z)$ is the solution to

$$
\Gamma_{r}=\phi\left(X_{\theta}^{t, x}, \tilde{P}_{\theta}^{t, p, a}\right)+\xi+\int_{r}^{T} f_{\xi}\left(s, X_{s}^{t, x}, \Gamma_{s}, Z_{s}\right) \mathrm{d} s-\int_{r}^{\theta} Z_{s} \mathrm{~d} W_{s}, t \leq r \leq \theta .
$$

Let $\Delta:=\Gamma-\hat{Y}, \delta Z=Z-\hat{Z}$, and $\delta f_{s}=f_{\xi}\left(s, X_{s}^{t, x}, \Gamma_{s}, Z_{s}\right)-f\left(s, X_{s}^{t, x}, \Gamma_{s}, Z_{s}\right)$ for $s \in[t, \theta]$. We then get

$$
\Delta_{r}:=\int_{r}^{\theta}\left(f\left(s, X_{s}^{t, x}, \Gamma_{s}, Z_{s}\right)-f\left(s, X_{s}^{t, x}, Y_{s}, Z_{s}\right)+\delta f_{s}\right) \mathrm{d} s-\int_{r}^{\theta} \delta Z_{s} \mathrm{~d} W_{s} .
$$

Classical energy estimates for BSDEs [21, 13] lead to

$$
\mathbb{E}\left[\sup _{r \in[t, \theta]}\left|\Delta_{r}\right|^{2}\right] \leq C \mathbb{E}\left[\int_{t}^{\theta}\left|\Delta_{s} \delta f_{s}\right| \mathrm{d} s\right] .
$$

Next, we compute

$$
\int_{t}^{\theta}\left|\Delta_{s} \delta f_{s}\right| \mathrm{d} s \leq \frac{1}{2 C} \sup _{s \in[t, \theta]}\left|\Delta_{s}\right|^{2}+2 C\left(\int_{t}^{\theta}\left|\delta f_{s}\right| \mathrm{d} s\right)^{2} .
$$

Combining the two previous inequalities, we obtain

$$
\mathbb{E}\left[\sup _{r \in[t, \theta]}\left|\Delta_{r}\right|^{2}\right] \leq 4 C^{2} \mathbb{E}\left[\left(\int_{t}^{\theta}\left|\delta f_{s}\right|\right)^{2}\right] .
$$

Using the Lipschitz property of $f$, we get from the definition of $f_{\xi}$,

$$
\left|\delta f_{s}\right| \leq L \xi
$$

which eventually leads to

$$
\mathbb{E}\left[\sup _{r \in[t, \theta]}\left|\Delta_{r}\right|^{2}\right] \leq C|\theta-t|^{2} \xi^{2}
$$

and concludes the proof.

Lemma 4.8. Let $0 \leq \tau<\theta \leq T$ and $\phi \in \mathcal{C}^{\infty}\left([0, T] \times \mathbb{R}^{d} \times[0,1]\right)$. For $(t, x, p) \in[\tau, \theta) \times$ $\mathbb{R}^{d} \times(0,1)$,

$$
\phi(t, x, p)-S^{a}(\tau, \theta, \phi(\theta, \cdot))(t, x, p)-(\theta-t) G^{a} \phi(t, x, p)=o(\theta-t),
$$

where $G^{a} \phi(t, x, p):=-\partial_{t} \phi(t, x, p)+F^{a}\left(t, x, p, \phi, D \phi, D^{2} \phi\right)$. 
Proof. We first observe that $S^{a}(\tau, \theta, \phi(\cdot))(t, x, p)=Y_{t}$ with $\left(Y^{a}, Z^{a}\right)$ the solution to

$$
Y_{r}=\Phi_{\theta}+\int_{r}^{\theta} f\left(s, X_{s}^{t, x}, Y_{s}, Z_{s}\right) \mathrm{d} s-\int_{r}^{\theta} Z_{s} \mathrm{~d} W_{s}
$$

with, for $t \leq s \leq \theta, \Phi_{s}=\phi\left(s, X_{s}^{t, x}, P_{s}^{t, p, \alpha}\right)$ and $\alpha:=a \mathbf{1}_{[0, \tau]}$. By a direct application of Itô's formula, we observe that

$$
\Phi_{r}=\Phi_{\theta}-\int_{r}^{\theta}\left\{\partial_{t} \phi+\mathcal{L}^{\alpha} \phi\right\}\left(s, X_{s}^{t, x}, P_{s}^{t, p, \alpha}\right) \mathrm{d} s-\int_{r}^{\theta} \mathfrak{Z}_{s} \mathrm{~d} W_{s}, \quad t \leq r \leq \theta
$$

where $\mathfrak{Z}_{s}:=\mathfrak{z}\left(X_{s}^{t, x}, D \phi\left(s, X_{s}^{t, x}, P_{s}^{t, p, a}\right), \alpha_{s}\right), t \leq s \leq \theta$.

For ease of exposition, we also introduce an auxiliary process $(\hat{Y}, \hat{Z})$ solution to

$$
\hat{Y}_{r}=\Phi_{\theta}+\int_{r}^{\theta} f\left(s, X_{s}^{t, x}, \Phi_{s}, \mathfrak{Z}_{s}\right) \mathrm{d} s-\int_{r}^{\theta} \hat{Z}_{s} \mathrm{~d} W_{s}, \quad t \leq r \leq \theta .
$$

Now, we compute

$$
\begin{aligned}
& \hat{Y}_{t}-\Phi_{t}+(\theta-t) G^{a} \phi(t, x, p) \\
& =\mathbb{E}\left[\int_{t}^{\theta}\left(\left\{\partial_{t} \phi\left(s, X_{s}^{t, x}, P_{s}^{t, p, a}\right)-\partial_{t} \phi(t, x, p)\right\}+\left\{F^{a} \phi\left(s, X_{s}^{t, x}, P_{s}^{t, p, a}\right)-F^{a} \phi(t, x, p)\right\}\right) \mathrm{d} s\right] .
\end{aligned}
$$

Using the smoothness of $\phi$, the Lipschitz property of $f$, and the following control,

$$
\mathbb{E}\left[\left|X_{s}^{t, x}-x\right|+\left|P_{s}^{t, p, \alpha}-p\right|\right] \leq C_{a}|\theta-t|^{\frac{1}{2}},
$$

we obtain

$$
\left|\hat{Y}_{t}-\Phi_{t}+(\theta-t) G^{a} \phi(t, x, p)\right| \leq C_{a, \phi}(\theta-t)^{\frac{3}{2}}
$$

We also have

$$
\hat{Y}_{r}-\Phi_{r}=\int_{r}^{\theta} G^{a} \phi\left(s, X_{s}^{t, x}, P_{s}^{t, p, \alpha}\right) \mathrm{d} s-\int_{r}^{\theta}\left(\hat{Z}_{s}-\mathfrak{Z}_{s}\right) \mathrm{d} W_{s} .
$$

Applying classical energy estimates for BSDEs, we obtain

$$
\begin{aligned}
\mathbb{E}\left[\sup _{r \in[t, \theta]}\left|\hat{Y}_{r}-\Phi_{r}\right|^{2}+\int_{t}^{\theta}\left|\hat{Z}_{s}-\mathfrak{Z}_{s}\right|^{2} \mathrm{~d} s\right] & \leq C \mathbb{E}\left[\left(\int_{t}^{\theta} \mid G^{a} \phi\left(s, X_{s}^{t, x}, P_{s}^{t, p, \alpha} \mid \mathrm{d} s\right)^{2}\right]\right. \\
& \leq C_{a, \phi}(\theta-t)^{2}
\end{aligned}
$$

where in the last line we used the smoothness of $\phi$ and the linear growth of $f$ and $\sigma$. We also observe that

$$
\hat{Y}_{r}-Y_{r}=\int_{r}^{\theta}\left\{\delta f_{s}+f\left(s, X_{s}^{t, x}, \hat{Y}_{s}, \hat{Z}_{s}\right)-f\left(s, X_{s}^{t, x}, Y_{s}, Z_{s}\right)\right\} \mathrm{d} s-\int_{r}^{\theta}\left\{\hat{Z}_{s}-Z_{s}\right\} \mathrm{d} W_{s},
$$


where $\delta f_{s}:=f\left(s, X_{s}^{t, x}, \Phi_{s}, \mathfrak{Z}_{s}\right)-f\left(s, X_{s}, \hat{Y}_{s}, \hat{Z}_{s}\right)$ for $t \leq s \leq \theta$. Once again, from classical energy estimates [21, 13], we obtain

$$
\left|\hat{Y}_{t}-Y_{t}\right|^{2} \leq C \mathbb{E}\left[\left(\int_{t}^{\theta} \delta f_{s} \mathrm{~d} s\right)^{2}\right]
$$

Using the Cauchy-Schwarz inequality and the Lipschitz property of $f$, we have

$$
\left|\hat{Y}_{t}-Y_{t}\right|^{2} \leq C(\theta-t) \mathbb{E}\left[\sup _{r \in[t, \theta]}\left|\hat{Y}_{r}-\Phi_{r}\right|^{2}+\int_{t}^{\theta}\left|\hat{Z}_{s}-\mathfrak{Z}_{s}\right|^{2} \mathrm{~d} s\right] .
$$

This last inequality, combined with (4.7), leads to

$$
\left|\hat{Y}_{t}-Y_{t}\right| \leq C(\theta-t)^{\frac{3}{2}}
$$

The proof is concluded by combining the above inequality with (4.6).

Finally, we can prove the following consistency property.

Proposition 4.9 (consistency). Let $\phi \in \mathcal{C}^{\infty}\left([0, T] \times \mathbb{R}^{d} \times[0,1]\right)$. For all $(t, x, p) \in[0, T) \times$ $\mathbb{R}^{d} \times(0,1)$, as $(|\pi|, \xi) \rightarrow 0$, we have

$$
\left|\frac{1}{t_{\pi}^{+}-t} \mathfrak{S}(\pi, t, x, p, \phi(t, x, p)+\xi, \phi(\cdot)+\xi)+\partial_{t} \phi-\mathcal{F}_{n}\left(t, x, p, \phi, D \phi, D^{2} \phi\right)\right| \rightarrow 0 .
$$

Proof. We first observe that by Lemma 4.7, it is sufficient to prove

$$
\left|\frac{1}{t_{\pi}^{+}-t} \mathfrak{S}(\pi, t, x, p, \phi(t, x, p), \phi(\cdot))+\partial_{t} \phi-\mathcal{F}_{n}\left(t, x, p, \phi, D \phi, D^{2} \phi\right)\right| \underset{|\pi| \downarrow 0}{\longrightarrow} 0 .
$$

We have that

$$
\begin{aligned}
& \left|\frac{1}{t_{\pi}^{+}-t} \mathfrak{S}(\pi, t, x, p, \phi(t, x, p), \phi(\cdot))+\partial_{t} \phi-\mathcal{F}_{n}\left(t, x, p, \phi, D \phi, D^{2} \phi\right)\right| \\
& =\left|\frac{1}{t_{\pi}^{+}-t}\left\{\phi(t, x, p)-\min _{a \in K} S^{a}\left(t_{\pi}^{-}, t_{\pi}^{+}, \phi\left(t_{\pi}^{+}, \cdot\right)\right)(t, x, p)\right\}-\max _{a \in K} G^{a}(t, x, p) \phi\right| \\
& \leq \max _{a \in K}\left|\frac{1}{t_{\pi}^{+}-t}\left\{\phi(t, x, p)-S^{a}\left(t_{\pi}^{-}, t_{\pi}^{+}, \phi\left(t_{\pi}^{+}, \cdot\right)\right)(t, x, p)\right\}-G^{a} \phi\right| .
\end{aligned}
$$

The proof is then concluded by applying Lemma 4.8 .

4.3. Properties of $S_{\delta}^{a}(\cdot)$. Let us first give some classical results on the finite difference scheme at the boundary, which is the classical finite difference scheme to approximate the superreplication price. Only partial proofs are given in this classical setting, and the proofs given later for the full scheme are easily adapted in this situation.

Proposition 4.10 (comparison theorem). Let $0 \leq t<s \leq T, \delta>0, h=s-t$ such that (2.30) to (2.32) is satisfied. Let $\left(u^{1}, u^{2}, v^{1}, v^{2}\right) \in \ell^{\infty}(\delta \mathbb{Z})^{4}$ such that $u_{k}^{1} \leq u_{k}^{2}$ for all $k \in \mathbb{Z}$. Then we have the following. 
1. For all $k \in \mathbb{Z}$ and $\left(v, \nabla, \nabla_{+}, \Delta\right) \in \mathbb{R}^{4}$, we have

$$
S_{b}\left(k, v, \nabla, \nabla_{+}, \Delta, u_{k}^{2}\right) \leq S_{b}\left(k, v, \nabla, \nabla_{+}, \Delta, u_{k}^{1}\right) .
$$

2. Assume that, for all $k \in \mathbb{Z}$,

$$
S_{b}\left(k, v_{k}^{1}, \nabla_{\delta} v_{k}^{1}, \nabla_{+, \delta} v_{k}^{1}, \Delta_{\delta} v_{k}^{1}, u_{k}^{1}\right) \leq 0 \leq S_{b}\left(k, v_{k}^{2}, \nabla_{\delta} v_{k}^{2}, \nabla_{+, \delta} v_{k}^{2}, \Delta_{\delta} v_{k}^{2}, u_{k}^{1}\right) .
$$

Then $v_{k}^{1} \leq v_{k}^{2}$ for all $k \in \mathbb{Z}$.

3. Assume that, for all $k \in \mathbb{Z}$,

$$
S_{b}\left(k, v_{k}^{1}, \nabla_{\delta} v_{k}^{1}, \nabla_{+, \delta} v_{k}^{1}, \Delta_{\delta} v_{k}^{1}, u_{k}^{1}\right)=0=S_{b}\left(k, v_{k}^{2}, \nabla_{\delta} v_{k}^{2}, \nabla_{+, \delta} v_{k}^{2}, \Delta_{\delta} v_{k}^{2}, u_{k}^{2}\right) .
$$

Then $v_{k}^{1} \leq v_{k}^{2}$ for all $k \in \mathbb{Z}$.

Proposition 4.11. Let $\pi, \delta>0$ such that (2.30) to (2.32) is satisfied for all $h=t_{j+1}-t_{j}, j=$ $0, \ldots, \kappa-1$. Let $V_{\pi, \delta}: \pi \times \delta \mathbb{Z} \rightarrow \mathbb{R}$ be the solution to

$$
\begin{aligned}
v_{k}^{\kappa}=g\left(x_{k}\right), & k \in \mathbb{Z}, \\
S_{b}\left(k, V_{k}^{j}, \nabla_{\delta} v_{k}^{j}, \nabla_{+, \delta} v_{k}^{j}, \Delta_{\delta} v_{k}^{j}, v_{k}^{j+1}\right)=0, & k \in \mathbb{Z}, 0 \leq j<\kappa .
\end{aligned}
$$

For all $k \in \mathbb{Z}$, let $\left(U_{\pi, \delta}\right)_{k}:=\frac{\left(V_{\pi, \delta}\right)_{k+1}-\left(V_{\pi, \delta}\right)_{k-1}}{2 \delta}$. Then

1. $\left(V_{\pi, \delta}, U_{\pi, \delta}\right) \in \ell^{\infty}(\delta \mathbb{Z})^{2}$ and their bound is independent of $\pi, \delta$;

2. $V_{\pi, \delta}$ converges uniformly on compact sets to $V$, the superreplication price of the contingent claim with payoff $g$.

Proof. We only show the first point, the second one is obtained by applying the arguments of [4], after proving monotonicity, stability, and consistency by the steps of subsection 4.4.

Since $g$ is bounded, it is easy to show that $V_{\pi, \delta}$ is also bounded independently of $\pi, \delta$, and the proof is similar to the proof of Proposition 4.14.

Since $g$ is Lipschitz-continuous, we get that $U_{\pi, \delta}(T, \cdot)$ is bounded. Using the Lipschitzcontinuity of $f$, one deduces easily that $U_{\pi, \delta}$ is a solution of

$$
\begin{aligned}
& u_{k}^{j}-u_{k}^{j+1}-h\left(-\mu \nabla_{+, \delta} u_{k}^{j}-\left(\frac{\sigma^{2}}{2}+\theta \frac{\delta^{2}}{h}\right) \Delta_{\delta} u_{k}^{j}-L-L\left|u_{k}^{j}\right|-L\left|\nabla_{\delta} u_{k}^{j}\right|\right) \geq 0, k \in \mathbb{Z}, 0 \leq j<\kappa, \\
& u_{k}^{j}-u_{k}^{j+1}-h\left(-\mu \nabla_{+, \delta} u_{k}^{j}-\left(\frac{\sigma^{2}}{2}+\theta \frac{\delta^{2}}{h}\right) \Delta_{\delta} u_{k}^{j}+L+L\left|u_{k}^{j}\right|+L\left|\nabla_{\delta} u_{k}^{j}\right|\right) \leq 0, k \in \mathbb{Z}, 0 \leq j<\kappa, \\
& u_{k}^{\kappa}=\frac{g\left(x_{k+1}\right)-g\left(x_{k-1}\right)}{2 \delta} \in[-L, L], k \in \mathbb{Z} .
\end{aligned}
$$

Again, comparison theorems can be proved, and it is now enough to show that there exists $(\underline{u}, \bar{u}) \in \ell^{\infty}(\pi \times \delta \mathbb{Z})^{2}$ which are bounded uniformly in $\pi, \delta$ such that

$$
\begin{aligned}
& \underline{u}_{k}^{j}-\underline{u}_{k}^{j+1}-h\left(-\mu \nabla_{+, \delta} \underline{u}_{k}^{j}-\left(\frac{\sigma^{2}}{2}+\theta \frac{\delta^{2}}{h}\right) \Delta_{\delta} \underline{u}_{k}^{j}-L-L\left|\underline{u}_{k}^{j}\right|-L\left|\nabla_{\delta} \underline{u}_{k}^{j}\right|\right) \leq 0, k \in \mathbb{Z}, 0 \leq j<\kappa, \\
& \bar{u}_{k}^{j}-\bar{u}_{k}^{j+1}-h\left(-\mu \nabla_{+, \delta} \bar{u}_{k}^{j}-\left(\frac{\sigma^{2}}{2}+\theta \frac{\delta^{2}}{h}\right) \Delta_{\delta} \bar{u}_{k}^{j}+L+L\left|\bar{u}_{k}^{j}\right|+L\left|\nabla_{\delta} \bar{u}_{k}^{j}\right|\right) \geq 0, k \in \mathbb{Z}, 0 \leq j<\kappa, \\
& \underline{u}_{k}^{\kappa} \leq-L, \bar{u}_{k}^{\kappa} \geq L, k \in \mathbb{Z} .
\end{aligned}
$$

Copyright (C) by SIAM. Unauthorized reproduction of this article is prohibited. 
We deal with $\underline{u}$ only, and obtain similar results for $\bar{u}$. One can easily show that $\underline{u}^{j}:=$ $1-(L+1) \prod_{k=j+1}^{\kappa} \frac{1}{1-h_{k} L}$, where $h_{k}:=t_{k}-t_{k-1}$ satisfies the requirements. Furthermore, one gets $\underline{u}^{j} \geq \underline{u}^{0} \geq 1-(L+1) 2^{\frac{T}{2 L}}$, thus one gets that $u$ is lower bounded by $1-(L+1) 2^{\frac{T}{2 L}}$.

We now turn to the proof of Proposition 2.15.

Proof of Proposition 2.15. First, $\underline{v}=B_{\delta}^{a}(s, t, \varphi(\cdot, 0))$ and $\bar{v}=B_{\delta}^{a}(s, t, \varphi(\cdot, 1))$ are uniquely defined in $\ell^{\infty}(\delta \mathbb{Z})$ by $(2.24)$; see Proposition 4.10 . We consider the following map:

$$
\begin{aligned}
\ell^{\infty}\left(\mathcal{G}_{\delta}^{a}\right) & \rightarrow \ell^{\infty}\left(\mathcal{G}_{\delta}^{a}\right), \\
v & \mapsto \psi(v),
\end{aligned}
$$

where $\psi(v)$ is defined, for $k \in \mathbb{Z}$ and $l \in\left\{1, \ldots, N_{a}-1\right\}$, by

$$
\begin{gathered}
\psi(v)_{k, l}=\frac{1}{1+\frac{h}{\delta} \mu+\sigma^{2} \frac{h}{\delta^{2}}+2 \theta}\left(\varphi\left(k \delta, \mathfrak{p}^{a}\left(p_{l}\right)\right)\right. \\
+\frac{h}{\delta} \mu v_{k+1, l+\operatorname{sgn}(a)}+\frac{\sigma^{2}}{2} \frac{h}{\delta^{2}}\left(v_{k+1, l+\operatorname{sgn}(a)}+v_{k-1, l-\operatorname{sgn}(a)}\right) \\
\left.+h f\left(t^{-}, k \delta, v_{k, l}, \frac{\sigma}{2 \delta}\left(v_{k+1, l+\operatorname{sgn}(a)}-v_{k-1, l-\operatorname{sgn}(a)}\right)\right)+\theta\left(v_{k+1, l+\operatorname{sgn}(a)}+v_{k-1, l-\operatorname{sgn}(a)}\right)\right), \\
\psi(v)_{k, 0}=\underline{v}_{k}, \quad \psi(v)_{k, N_{a}}=\bar{v}_{k} .
\end{gathered}
$$

Notice that $v$ is a solution to (2.26)-(2.27) if and only if $v$ is a fixed point of $\psi$. It is now enough to show that $\psi$ maps $\ell^{\infty}\left(\mathcal{G}_{\delta}^{a}\right)$ into $\ell^{\infty}\left(\mathcal{G}_{\delta}^{a}\right)$ and is contracting. If $v \in \ell^{\infty}\left(\mathcal{G}_{\delta}^{a}\right)$, by boundedness of $\varphi, \underline{v}$, and $\bar{v}$, it is clear that $\psi(v)$ is bounded. If $v^{1}, v^{2} \in \ell^{\infty}\left(\mathcal{G}_{\delta}^{a}\right)^{2}$, we have, for all $k \in \mathbb{Z}$ and $1 \leq l \leq N_{a}-1$,

$$
\left|\psi\left(v^{1}\right)_{k, l}-\psi\left(v^{2}\right)_{k, l}\right| \leq \frac{\frac{h}{\delta} \mu+\sigma^{2} \frac{h}{\delta^{2}}+2 \theta+h L+\frac{h L}{\delta}}{1+\frac{h}{\delta} \mu+\sigma^{2} \frac{h}{\delta^{2}}+2 \theta}\left|v^{1}-v^{2}\right|_{\infty} .
$$

Since $\delta \leq 1$ by assumption (2.30), one has $h L+\frac{h L}{\delta} \leq 2 \frac{h L}{\delta} \leq 4 \theta$, thus,

$$
\left|\psi\left(v^{1}\right)-\psi\left(v^{2}\right)\right|_{\infty} \leq \frac{4 \theta+\frac{h}{\delta} \mu+\sigma^{2} \frac{h}{\delta^{2}}+2 \theta}{1+\frac{h}{\delta} \mu+\sigma^{2} \frac{h}{\delta^{2}}+2 \theta}\left|v^{1}-v^{2}\right|_{\infty} .
$$

Since $4 \theta<1$ by assumption (2.31) and the function $x \mapsto \frac{4 \theta+x}{1+x}$ is increasing on $[0, \infty)$ with limit 1 when $x \rightarrow+\infty$, this proves that $\psi$ is a contracting mapping.

Proof of Proposition 2.16. Let $\varphi^{1}, \varphi^{2}$ as stated in the proposition.

1. We have, for $k \in \mathbb{Z}$ and $0<l<N_{\delta}^{a}$,

$$
\begin{aligned}
& S\left(k, l, v, q, q_{+}, A, \varphi^{2}\right)-S\left(k, l, v, q, q_{+}, A, \varphi^{1}\right) \\
& =\left(\varphi^{1}-\varphi^{2}\right)\left(x_{k}, \mathfrak{p}^{a}\left(p_{l}\right)\right) \leq 0 .
\end{aligned}
$$

2. We assume here that $a>0$. For $k \in \mathbb{Z}$, let $M_{k}=\max _{0 \leq l \leq N_{\delta}^{a}}\left(v_{k+l, l}^{1}-v_{k+l, l}^{2}\right)<\infty$ (if $a<0$, we have to consider $\left.\max _{0 \leq l \leq N_{\delta}^{a}}\left(v_{k-l, l}^{1}-v_{k-l, l}^{2}\right)\right)$. We want to prove that 
$M_{k} \leq 0$ for all $k$. Assume to the contrary that there exists $k \in \mathbb{Z}$ such that $M_{k}>0$ and, thus, there exists $0 \leq l \leq N_{\delta}^{a}$ such that $v_{k+l, l}^{1}-v_{k+l, l}^{2}=M_{k}>0$. Since $v_{k, 0}^{1} \leq v_{k, 0}^{2}$ and $v_{k+N_{\delta}^{a}, N_{\delta}^{a}}^{1} \leq v_{k+N_{\delta}^{a}, N_{\delta}^{a}}^{2}$, we have $0<l<N_{\delta}^{a}$. Moreover, using (2.34), rearranging the terms, using the fact that $f$ is nonincreasing with respect to its third variable and Lipschitz-continuous, and that $M_{k}$ is attained for $l$,

$$
\begin{aligned}
(1+2 \theta) M_{k} & \leq \frac{h L}{2 \delta}\left|v_{k+l+1, l+1}^{2}-v_{k+l+1, l+1}^{1}\right|-\theta\left(v_{k+l+1, l+1}^{2}-v_{k+l+1, l+1}^{1}\right) \\
& +\frac{h L}{2 \delta}\left|v_{k+l-1, l-1}^{2}-v_{k+l-1, l-1}^{1}\right|-\theta\left(v_{k+l-1, l-1}^{2}-v_{k+l-1, l-1}^{1}\right) .
\end{aligned}
$$

For $j \in\{l-1, l+1\}$, we observe that

$$
\frac{h L}{2 \delta}\left|v_{k+j, j}^{2}-v_{k+j, j}^{1}\right|-\theta\left(v_{k+j, j}^{2}-v_{k+j, j}^{1}\right) \leq\left(\frac{h L}{2 \delta}+\theta\right) M_{k} .
$$

Indeed, if $v_{k+j, j}^{2} \geq v_{k+j, j}^{1}$, then

$$
\frac{h L}{2 \delta}\left|v_{k+j, j}^{2}-v_{k+j, j}^{1}\right|-\theta\left(v_{k+j, j}^{2}-v_{k+j, j}^{1}\right)=\left(\frac{h L}{2 \delta}-\theta\right)\left(v_{k+j, j}^{2}-v_{k+j, j}^{1}\right) \leq 0,
$$

since $\frac{h L}{2 \delta} \leq \theta$. Otherwise, if $v_{k+j, j}^{2}<v_{k+j, j}^{1}$,

$$
\begin{aligned}
\frac{h L}{2 \delta}\left|v_{k+j, j}^{2}-v_{k+j, j}^{1}\right|-\theta\left(v_{k+j, j}^{2}-v_{k+j, j}^{1}\right) & =\left(\frac{h L}{2 \delta}+\theta\right)\left(v_{k+j, j}^{1}-v_{k+j, j}^{2}\right) \\
& \leq\left(\frac{h L}{2 \delta}+\theta\right) M_{k}
\end{aligned}
$$

Inserting (4.9) into (4.8), we get

$$
(1+2 \theta) M_{k} \leq 2\left(\frac{h L}{2 \delta}+\theta\right) M_{k}
$$

Thus,

$$
\left(1-\frac{h L}{\delta}\right) M_{k} \leq 0
$$

which is a contradiction to $M_{k}>0$ since $\frac{h L}{\delta} \leq 2 \theta<\frac{1}{2}$.

3. Let $v^{i}=S_{\delta}^{a}\left(s, t, \varphi^{i}\right)$ for $i=1,2$. Since $\underline{\varphi}^{1} \leq \underline{\varphi}^{2}$ and $\bar{\varphi}^{1} \leq \bar{\varphi}^{2}$, we get by Proposition 4.10 that $v_{k, 0}^{1} \leq v_{k, 0}^{2}$ and $v_{k, N_{\delta}^{a}}^{1} \leq v_{k, N_{\delta}^{a}}^{2} \overline{\text { for }}$ all $\bar{k} \in \mathbb{Z}$.

By monotonicity, we get, for all $k \in \mathbb{Z}$ and $0<l<N_{\delta}^{a}$,

$$
\begin{aligned}
S\left(k, l, v_{k, l}^{1}, \nabla_{\delta}^{a} v_{k, l}^{1}, \nabla_{+, \delta}^{a} v_{k, l}^{1}, \Delta_{\delta}^{a} v_{k, l}^{1}, \varphi^{2}\right) \\
\\
\leq S\left(k, l, v_{k, l}^{1}, \nabla_{\delta}^{a} v_{k, l}^{1}, \nabla_{+, \delta}^{a} v_{k, l}^{1}, \Delta_{\delta}^{a} v_{k, l}^{1}, \varphi^{1}\right) .
\end{aligned}
$$

Copyright (C) by SIAM. Unauthorized reproduction of this article is prohibited. 
Moreover,

$$
\begin{aligned}
S\left(k, l, v_{k, l}^{1}, \nabla_{\delta}^{a} v_{k, l}^{1},\right. & \left.\nabla_{+, \delta}^{a} v_{k, l}^{1}, \Delta_{\delta}^{a} v_{k, l}^{1}, \varphi^{1}\right) \\
& =S\left(k, l, v_{k, l}^{2}, \nabla_{\delta}^{a} v_{k, l}^{2}, \nabla_{+, \delta}^{a} v_{k, l}^{2}, \Delta_{\delta}^{a} v_{k, l}^{2}, \varphi^{2}\right)=0,
\end{aligned}
$$

so that

$$
\begin{aligned}
S\left(k, l, v_{k, l}^{1}, \nabla_{\delta}^{a} v_{k, l}^{1},\right. & \left.\nabla_{+, \delta}^{a} v_{k, l}^{1}, \Delta_{\delta}^{a} v_{k, l}^{1}, \varphi^{2}\right) \\
& \leq S\left(k, l, v_{k, l}^{2}, \nabla_{\delta}^{a} v_{k, l}^{2}, \nabla_{+, \delta}^{a} v_{k, l}^{2}, \Delta_{\delta}^{a} v_{k, l}^{2}, \varphi^{2}\right)
\end{aligned}
$$

and the proof is concluded applying the previous point.

To conclude this subsection, we prove Proposition 2.17.

Proof of Proposition 2.17. For ease of notation, we set

$$
\begin{aligned}
e(l) & :=e^{-4 \frac{\mathfrak{a}(a, \delta)^{2}}{\sigma^{2}} C(h, \delta) l\left(N^{a}-l\right)}, \\
e^{\star} & :=\min _{x \in\left[0, N_{\delta}^{a}\right]} e(x)=e\left(N_{\delta}^{a} / 2\right)=e^{-\frac{\mathfrak{a}(a, \delta)^{2}}{\sigma^{2}} C(h, \delta)\left(N_{\delta}^{a}\right)^{2}}=e^{-\frac{C(h, \delta)}{\delta^{2}}}, \\
B & :=\left|\left(v_{\cdot, 0}^{1}-v_{\cdot, 0}^{2}\right)^{+}\right|_{\infty}+\left|\left(v_{\cdot, N_{\delta}^{a}}^{1}-v_{\cdot, N_{\delta}^{a}}^{2}\right)^{+}\right|_{\infty} .
\end{aligned}
$$

By the comparison theorem, it is enough to show that $w \in \ell^{\infty}\left(\mathcal{G}_{\delta}^{a}\right)$ defined by

$$
w_{k, l}:=v_{k, l}^{2}+B e(l)
$$

satisfies $w_{k, 0} \geq v_{k, 0}^{1}, w_{k, N_{\delta}^{a}} \geq v_{k, N_{\delta}^{a}}^{1}$, and $S\left(k, l, w_{k, l}, \nabla_{\delta}^{a} w_{k, l}, \nabla_{+, \delta}^{a} w_{k, l}, \Delta_{\delta}^{a} w_{k, l}, u\right) \geq 0$ for all $k \in \mathbb{Z}$ and $0<l<N_{\delta}^{a}$.

The boundary conditions are easily checked: if $k \in \mathbb{Z}$ and $l \in\left\{0, N_{a}\right\}$, we have, since $e(0)=e\left(N_{a}\right)=1$,

$$
w_{k, l}=v_{k, l}^{2}+B \geq v_{k, l}^{2}+\left(v_{k, l}^{1}-v_{k, l}^{2}\right)^{+} \geq v_{k, l}^{1} .
$$

For $k \in \mathbb{Z}, 1 \leq l \leq N_{\delta}^{a}-1$, we prove $S\left(k, l, w_{k, l}, \nabla_{\delta}^{a} w_{k, l}, \nabla_{+, \delta}^{a} w_{k, l}, \Delta_{\delta}^{a} w_{k, l}, u\right) \geq 0$.

By definition (2.28), inserting $\pm h f\left(t^{-}, e^{k \delta}, v_{k, l}^{2}, \frac{1}{2 \delta}\left(w_{k+1, l+\operatorname{sgn}(a)}-w_{k-1, l-\operatorname{sgn}(a)}\right)\right)$, since $S\left(k, l, v_{k, l}^{2}, \nabla_{\delta}^{a} v_{k, l}^{2}, \nabla_{+, \delta}^{a} v_{k, l}^{2}, \Delta_{\delta}^{a} v_{k, l}^{2}, u\right) \geq 0$ and since $f$ is nonincreasing with respect to its third variable and Lipschitz-continuous with respect to its fourth variable, we have

$$
\begin{aligned}
& S\left(k, l, w_{k, l}, \nabla_{\delta}^{a} w_{k, l}, \nabla_{+, \delta}^{a} w_{k, l}, \Delta_{\delta}^{a} w_{k, l}, u\right) \\
& \geq B\left[\left(1+\mu \frac{h}{\delta}+\sigma^{2} \frac{h}{\delta^{2}}+2 \theta\right) e(l)-\left(\mu \frac{h}{\delta}+\frac{\sigma^{2}}{2} \frac{h}{\delta^{2}}+\theta\right) e(l+\operatorname{sgn}(a))\right. \\
& \left.\quad-\left(\frac{\sigma^{2}}{2} \frac{h}{\delta^{2}}+\theta\right) e(l-\operatorname{sgn}(a))-\frac{h L}{2 \delta}|e(l+\operatorname{sgn}(a))-e(l-\operatorname{sgn}(a))|\right] .
\end{aligned}
$$

We have $|e(l+\operatorname{sgn}(a))-e(l-\operatorname{sgn}(a))| \leq 1-e^{\star}$, thus,

$$
\begin{aligned}
& S\left(k, l, w_{k, l}, \nabla_{\delta}^{a} w_{k, l}, \nabla_{+, \delta}^{a} w_{k, l}, \Delta_{\delta}^{a} w_{k, l}, u\right) \\
& \geq B\left[\left(1+\mu \frac{h}{\delta}+\sigma^{2} \frac{h}{\delta^{2}}+2 \theta+\frac{h L}{2 \delta}\right) e^{\star}-\mu \frac{h}{\delta}-\sigma^{2} \frac{h}{\delta^{2}}-2 \theta-\frac{h L}{2 \delta}\right] .
\end{aligned}
$$

Copyright (C) by SIAM. Unauthorized reproduction of this article is prohibited. 
It is thus enough to have

$$
\left(1+\mu \frac{h}{\delta}+\sigma^{2} \frac{h}{\delta^{2}}+2 \theta+\frac{h L}{2 \delta}\right) e^{\star}-\mu \frac{h}{\delta}-\sigma^{2} \frac{h}{\delta^{2}}-2 \theta-\frac{h L}{2 \delta} \geq 0,
$$

and one can easily check that this is the case with our choice of $C(h, \delta)$.

It remains to prove $(2.36)$. Since $\ln (1+x)>x-\frac{x^{2}}{2}$ for all $x>0$, we have, by $(2.32)$,

$$
\begin{aligned}
C(h, \delta) & >\frac{1}{\delta^{2}}\left(\frac{1}{\mu \frac{h}{\delta}+\sigma^{2} \frac{h}{\delta^{2}}+2 \theta+\frac{h L}{2 \delta}}-\frac{1}{2} \frac{1}{\left(\mu \frac{h}{\delta}+\sigma^{2} \frac{h}{\delta^{2}}+2 \theta+\frac{h L}{2 \delta}\right)^{2}}\right) \\
& =\frac{1}{\left(\mu+\frac{L}{2}\right) \delta h+\sigma^{2} h+2 \theta \delta^{2}}-\frac{1}{2\left(\left(\mu+\frac{L}{2}\right) h+\sigma^{2} \frac{h}{\delta}+2 \delta \theta\right)^{2}} \\
& \geq \frac{1}{\left(\left(\mu+\frac{L}{2}\right) M+2 \theta M^{2}\right) h^{2}+\sigma^{2} h}-\frac{1}{2\left(\left((1+2 \theta) \mu+\frac{L}{2}\right) h+\frac{\sigma^{2}}{M}\right)^{2}} \\
& \geq \frac{1}{\left(\left(\mu+\frac{L}{2}\right) M+2 \theta M^{2}\right) h^{2}+\sigma^{2} h}-\frac{M^{2}}{2 \sigma^{4}} .
\end{aligned}
$$

4.4. Proof of Theorem 2.21. We first show that the numerical scheme is consistent with the boundary conditions. For any discretization parameters $\pi, \delta$, we define $V_{\pi, \delta}: \pi \times \delta \mathbb{Z} \rightarrow \mathbb{R}$ as the solution to the following system, with $S_{b}$ from (2.25):

$$
\begin{aligned}
S_{b}\left(k, v_{k}^{j}, \nabla_{\delta} v_{k}^{j}, \nabla_{+, \delta} v_{k}^{j}, \Delta_{\delta} v_{k}^{j}, v_{k}^{j+1}\right) & =0, k \in \mathbb{Z}, 0 \leq j<\kappa, \\
v_{k}^{\kappa} & =g\left(x_{k}\right), k \in \mathbb{Z},
\end{aligned}
$$

where $v_{k}^{j}:=v\left(t_{j}, x_{k}\right)$ for $0 \leq j \leq \kappa$ and $k \in \mathbb{Z}$. We set $\left(U_{\pi, \delta}\right)_{k}^{j}:=\nabla_{\delta}\left(V_{\pi, \delta}\right)_{k}^{j}=\frac{1}{2 \delta}\left(\left(V_{\pi, \delta}\right)_{k+1}^{j}-\right.$ $\left.\left(V_{\pi, \delta}\right)_{k-1}^{j}\right)$. By Proposition 4.11, $V_{\pi, \delta}$ and $U_{\pi, \delta}$ are bounded, uniformly in $\pi, \delta$, and, by [4], $V_{\pi, \delta}$ converges to $V$ (the superreplication price) uniformly on compact sets as $|\pi|, \delta \rightarrow 0$.

Proposition 4.12. There exist constants $K_{1}, K_{2}, K_{3}>0$ such that, for all discretization parameters $\pi, \delta$ with $|\pi|$ small enough, we have, for $\left(t_{j}, x_{k}, p\right) \in \pi \times \delta \mathbb{Z} \times[0,1]$,

$$
\begin{gathered}
p V_{\pi, \delta}\left(t_{j}, x_{k}\right)-K_{1}\left(T-t_{j}\right) \leq v_{n, \pi, \delta}\left(t_{j}, x_{k}, p\right) \leq p V_{\pi, \delta}\left(t_{j}, x_{k}\right)+K_{1}\left(T-t_{j}\right), \\
p V_{\pi, \delta}\left(t_{j}, x_{k}\right)-\left(1-e^{-K_{2} p}\right)\left(1-e^{-K_{2}(1-p)}\right) \leq v_{n, \pi, \delta}\left(t_{j}, x_{k}, p\right) \\
\leq p V_{\pi, \delta}\left(t_{j}, x_{k}\right)+\left(1-e^{-K_{2} p}\right)\left(1-e^{-K_{2}(1-p)}\right) .
\end{gathered}
$$

Proof. We only prove, by backward induction, the lower bounds, while the proof of the upper bounds is similar.

For $0 \leq j \leq \kappa, k \in \delta \mathbb{Z}$, and $0 \leq l \leq N_{\delta}^{a}$, we set $V_{k}^{j}:=V_{\pi, \delta}\left(t_{j}, x_{k}\right)$ and $U_{k}^{j}:=U_{\pi, \delta}\left(t_{j}, x_{k}\right)$. For $\epsilon \in\{0,1\}$, we define ${ }^{\epsilon} w\left(t_{j}, x_{k}, p\right):=p V_{k}^{j}-{ }^{\epsilon} c\left(t_{j}, p\right)$ with ${ }^{\epsilon} c\left(t_{j}, p\right):=\epsilon K_{1}\left(T-t_{j}\right)+$ $(1-\epsilon)\left(1-e^{-K_{2} p}\right)\left(1-e^{-K_{2}(1-p)}\right)$, and $\epsilon_{w_{k, l}^{j}}^{j}=\epsilon_{w}\left(t_{j}, x_{k}, p_{l}\right),{ }^{\epsilon} c_{l}^{j}={ }^{\epsilon} c\left(t_{j}, p_{l}\right), p_{l} \in \Gamma_{\delta}^{a}$. The proof now proceeds in two steps.

Copyright (C) by SIAM. Unauthorized reproduction of this article is prohibited. 
1. First, we have ${ }^{\epsilon} w\left(T, x_{k}, p\right) \leq p V_{\pi, \delta}\left(T, x_{k}\right)=p g\left(x_{k}\right)=v_{n, \pi, \delta}\left(T, x_{k}, p\right)$ on $\delta \mathbb{Z} \times[0,1]$. Suppose that, for $0 \leq j<\kappa$ on $\delta \mathbb{Z} \times[0,1]$, we have

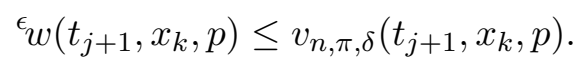

We want to prove on $\delta \mathbb{Z} \times[0,1]$ that

$$
\epsilon_{w}\left(t_{j}, x_{k}, p\right) \leq v_{n, \pi, \delta}\left(t_{j}, x_{k}, p\right)
$$

Since ${ }^{\epsilon} w$ is convex in $p,{ }^{\epsilon} w\left(t_{j}, x_{k}, \cdot\right) \leq \mathcal{I}_{\delta}^{a}\left(\epsilon w_{k,}^{j}\right)$ on $[0,1]$. By definition, we have

$$
v_{n, \pi, \delta}\left(t_{j}, x_{k}, p\right)=\min _{a \in K} \mathcal{I}_{\delta}^{a}\left(S_{\delta}^{a}\left(t_{j+1}, t_{j}, v_{n, \pi, \delta}\left(t_{j+1}, \cdot\right)\right)\right)\left(x_{k}, p\right)
$$

We are thus going to prove

$$
\epsilon_{w_{k, l}^{j}}^{j} \leq S_{\delta}^{a}\left(t_{j+1}, t_{j}, v_{n, \pi, \delta}\left(t_{j+1}, \cdot\right)\right)\left(t_{j}, x_{k}, p_{l}\right)
$$

for all $a \in K$ and all $k \in \mathbb{Z}, 0 \leq l \leq N_{\delta}^{a}$. For $a \in K$, by the induction hypothesis, ${ }^{\epsilon} w\left(t_{j+1}, \cdot\right) \leq$ $v_{n, \pi, \delta}\left(t_{j+1}, \cdot\right)$, so if we are able to get

$$
\begin{gathered}
S_{b}\left(k, \underline{\epsilon}_{k}, \nabla_{\delta} \underline{w}_{k}, \nabla_{+, \delta} \underline{w}_{k}, \Delta_{\delta} \underline{\epsilon}_{k}, \underline{w}_{k, 0}^{j+1}\right) \leq 0, k \in \mathbb{Z}, \\
S_{b}\left(k, \bar{\epsilon}_{k}, \nabla_{\delta} \bar{\epsilon}_{k}, \nabla_{+, \delta} \bar{w}_{k}, \Delta_{\delta} \bar{w}_{k},{ }^{\epsilon} w_{k, N_{\delta}^{a}}^{j+1}\right) \leq 0, k \in \mathbb{Z}, \\
S\left(k, l,{ }^{\epsilon} w_{k, l}, \nabla_{\delta}^{a \epsilon} w_{k, l}, \nabla_{+, \delta}^{a}{ }^{\epsilon} w_{k, l}, \Delta_{\delta}^{a \epsilon} w_{k, l},{ }^{\epsilon} w\left(t_{j+1}, \cdot\right)\right) \leq 0, k \in \mathbb{Z}, 0<l<N_{\delta}^{a},
\end{gathered}
$$

where $\underline{\epsilon}_{k}^{j}={ }^{\epsilon} w\left(t_{j}, x_{k}, 0\right), \bar{\epsilon}_{k}^{j}={ }^{\epsilon} w\left(t_{j}, x_{k}, 1\right)$, we obtain that (4.11) holds true by the comparison result in Proposition 2.16, which concludes the proof. We now proceed with the proof of (4.12), (4.13), and (4.14).

2(a). Now, observe that $\underline{\epsilon}_{k}^{j}=-\epsilon K_{1}\left(T-t_{j}\right)$ for $k \in \mathbb{Z}$. We have, since $f\left(t_{j}, x_{k}, 0,0\right)=0$ and $f$ is nonincreasing in its third variable,

$$
S_{b}\left(k, \underline{\epsilon}_{k}, \nabla_{\delta} \underline{w}_{k}, \nabla_{+, \delta} \underline{w}_{k}, \Delta_{\delta} \underline{w}_{k},{ }^{\epsilon} w_{k, 0}^{j+1}\right)=-\epsilon K h-h f\left(t_{j}, x_{k},-\epsilon K\left(T-t_{j}\right), 0\right) \leq 0 .
$$

2(b). We have that $\bar{\epsilon}_{k}^{j}=V_{k}^{j}-\epsilon K_{1}\left(T-t_{j}\right)$, for $k \in \mathbb{Z}$. Since

$$
f\left(t_{j}, x_{k}, V_{k}^{j}-\epsilon K_{1}\left(T-t_{j}\right), U_{k}^{j}\right) \geq f\left(t_{j}, x_{k}, V_{k}^{j}, U_{k}^{j}\right),
$$

and by the definition of $V_{\pi, \delta}$,

$$
\begin{aligned}
S_{b}\left(k, \bar{\epsilon}_{k}, \nabla_{\delta} \bar{\epsilon}_{k}, \nabla_{+, \delta} \bar{\epsilon}_{k}, \Delta_{\delta} \bar{\epsilon}_{k},{ }^{\epsilon} w_{k, N_{\delta}^{a}}^{j+1}\right) & =-\epsilon K h+S_{b}\left(k, V_{k}^{j}, \nabla_{\delta} V_{k}^{j}, \nabla_{+, \delta} V_{k}^{j}, \Delta_{\delta} V_{k}^{j}\right) \\
& \leq-\epsilon K h \leq 0 .
\end{aligned}
$$

Copyright (C) by SIAM. Unauthorized reproduction of this article is prohibited. 
2(c). We now prove (4.14). Let $k \in \mathbb{Z}, 0<l<N_{\delta}^{a}$. We have, by the definition (2.28) of $S$,

$$
\begin{aligned}
& S\left(k, l,{ }^{\epsilon} w_{k, l}^{j}, \nabla_{\delta}^{a \epsilon} w_{k, l}^{j}, \nabla_{+, \delta}^{a}{ }^{\epsilon} w_{k, l}^{j}, \Delta_{\delta}^{a \epsilon} w_{k, l}^{j},{ }^{\epsilon} w\left(t_{j+1}, \cdot\right)\right) \\
&= \epsilon_{k, l}^{j}-{ }^{\epsilon} w\left(t_{j+1}, x_{k}, \mathfrak{p}^{a}\left(p_{l}\right)\right) \\
&+h \widehat{F}\left(t, k \delta,{ }^{\epsilon} w_{k, l}^{j}, \nabla_{\delta}^{a \epsilon} w_{k, l}^{j}, \nabla_{+, \delta}^{a}{ }^{\epsilon} w_{k, l}^{j}, \Delta_{\delta}^{a \epsilon} w_{k, l}^{j}\right) \\
& \leq-{ }^{\epsilon} c_{k, l}^{j}+\mu \frac{\mathfrak{a}(a, \delta)}{\sigma} h V_{k}^{j+1}+{ }^{\epsilon} c\left(t_{j+1}, x_{k}, \mathfrak{p}^{a}\left(p_{l}\right)\right) \\
&-p_{l} h \widehat{F}\left(t, x_{k}, V_{k}^{j}, U_{k}^{j}, \nabla_{+, \delta} V_{k}^{j}, \Delta_{\delta} V_{k}^{j}\right) \\
&+h \widehat{F}\left(t, x_{k}, p_{l} V_{k}^{j}, \nabla_{\delta}^{a \epsilon} w_{k, l}^{j}, \nabla_{+, \delta}^{a} w_{k, l}^{j}, \Delta_{\delta}^{a \epsilon} w_{k, l}^{j}\right),
\end{aligned}
$$

where we have used (4.10) and $f\left(t, x_{k},{ }^{\epsilon} w_{k, l}^{j}, \sigma \nabla_{\delta}^{a \epsilon} w_{k, l}^{j}\right) \geq f\left(t, x_{k}, p_{l} V_{k}^{j}, \sigma \nabla_{\delta}^{a \epsilon} w_{k, l}^{j}\right)$.

By adding $\pm p_{l} h f\left(t_{j}, x_{k}, p_{l} V_{k}^{j}, \sigma \nabla_{\delta}^{a \epsilon} w_{k, l}^{j}\right)$, using the Lipschitz continuity of $f$, and

$$
\nabla_{\delta}^{a \epsilon} w_{k, l}^{j}=p_{l} U_{k}^{j}+\frac{\mathfrak{a}(a, \delta)}{2 \sigma}\left(V_{k+1}^{j}+V_{k-1}^{j}\right)+\frac{1}{2 \delta}\left({ }^{\epsilon}{ }^{j}{ }_{l-\operatorname{sgn}(a)}^{j}-{ }^{\epsilon} c_{l+\operatorname{sgn}(a)}^{j}\right)
$$

we get, by the definition $(2.23)$ of $\widehat{F}$,

$$
\begin{aligned}
& S\left(k, l,{ }^{\epsilon} w_{k, l}, \nabla_{\delta}^{a \epsilon} w_{k, l}^{j}, \nabla_{+, \delta}^{a}{ }^{\epsilon} w_{k, l}^{j}, \Delta_{\delta}^{a \epsilon} w_{k, l}^{j},{ }^{\epsilon} w^{j}\left(t_{j+1}, \cdot\right)\right) \\
& \leq h \frac{\mathfrak{a}(a, \delta)}{\sigma} \mu\left(V_{k}^{j+1}-V_{k+1}^{j}\right)-h \sigma \mathfrak{a}(a, \delta) U_{k}^{j}-2 \theta \frac{\mathfrak{a}(a, \delta)}{\sigma} \delta^{2} U_{k}^{j} \\
&+2 h L p_{l}\left(1-p_{l}\right)\left(V_{k}^{j}+\left|U_{k}^{j}\right|\right)+h L \frac{|\mathfrak{a}(a, \delta)|}{2 \sigma}\left(V_{k+1}^{j}+V_{k-1}^{j}\right) \\
&-\left({ }^{\epsilon}{ }^{j}{ }_{l}^{j}-{ }^{\epsilon} c\left(t_{j+1}, \mathfrak{p}^{a}\left(p_{l}\right)\right)-\mu h \nabla_{+, \delta}^{a}{ }^{\epsilon} c_{l}^{j}-\left(\frac{\sigma^{2}}{2} h+\theta \delta^{2}\right) \Delta_{\delta}^{a \epsilon} c_{l}^{j}\right) \\
&+h L\left|\nabla_{\delta}^{a \epsilon} c_{l}^{j}\right| .
\end{aligned}
$$

Since $|\mathfrak{a}(a, \delta)| \leq \max \{|a|, a \in K\} \leq n$ and $V$ and $U$ are bounded uniformly in $h, \delta$ (see Proposition 4.11), there exists a constant $K_{n, \theta, M, L}>0$ such that

$$
\begin{aligned}
& h \frac{\mathfrak{a}(a, \delta)}{\sigma} \mu\left(V_{k}^{j+1}-V_{k+1}^{j}\right)-h \sigma \mathfrak{a}(a, \delta) U_{k}^{j}-2 \theta \frac{\mathfrak{a}(a, \delta)}{\sigma} \delta^{2} U_{k}^{j} \\
& +2 h L p_{l}\left(1-p_{l}\right)\left(V_{k}^{j}+\left|U_{k}^{j}\right|\right)+h L \frac{|\mathfrak{a}(a, \delta)|}{2 \sigma}\left(V_{k+1}^{j}+V_{k-1}^{j}\right) \leq h K_{n, \theta, M, L} .
\end{aligned}
$$

When $\epsilon=1$, the terms of the last three lines all vanish except the first one, and $c_{l}^{j}-c\left(t_{j+1}, p_{l}-\right.$ $\left.\mu \frac{\mathfrak{a}(a, \delta)}{\sigma} h\right)=K_{1} h$. Thus we get

$$
S\left(k, l,{ }^{\epsilon} w_{k, l}, \nabla_{\delta}^{a \epsilon} w_{k, l}, \nabla_{+, \delta}^{a} w_{k, l}, \Delta_{\delta}^{a \epsilon} w_{k, l},{ }^{\epsilon} w\left(t_{j+1}, \cdot\right)\right) \leq h\left(-K_{1}+K_{n, \theta, M, L}\right) .
$$

Hence, chosing $K_{1}$ large enough gives the result.

We now deal with the case $\epsilon=0$. By Taylor expansions of $\epsilon_{c}$ around $\left(t_{j}, p_{l}\right)$, we get

$$
\begin{aligned}
& S\left(k, l,{ }^{\epsilon} w_{k, l}, \nabla_{\delta}^{a \epsilon} w_{k, l}^{j}, \nabla_{+, \delta}^{a} \epsilon_{k, l}^{j}, \Delta_{\delta}^{a \epsilon} w_{k, l}^{j},{ }^{\epsilon} w^{j}\left(t_{j+1}, \cdot\right)\right) \\
& \leq h K_{n, \theta, M, L}+h L \frac{|\mathfrak{a}(a, \delta)|}{\sigma}\left|\partial_{p}{ }^{\epsilon} c\left(t_{j}, p_{l}\right)\right|+h \partial_{t}{ }^{\epsilon} c\left(t_{j}, p_{l}\right)+h \frac{\mathfrak{a}(a, \delta)^{2}}{2} \partial_{p p}^{2} \epsilon\left(t_{j}, p_{l}\right)+h \varepsilon\left(h ; K_{2}\right)
\end{aligned}
$$

Copyright (C) by SIAM. Unauthorized reproduction of this article is prohibited. 
with $\lim _{h \rightarrow 0} \varepsilon\left(h ; K_{2}\right)=0$. By the definition of ${ }^{\epsilon} c$, we get, for $h_{0}>0$ to be fixed later on and $h \in\left[0, h_{0}\right]$,

$$
\begin{aligned}
& S\left(k, l,{ }^{\epsilon} w_{k, l}, \nabla_{\delta}^{a \epsilon} w_{k, l}^{j}, \nabla_{+, \delta}^{a} \omega_{k, l}^{j}, \Delta_{\delta}^{a \epsilon} w_{k, l}^{j}, \epsilon^{j} w^{j}\left(t_{j+1}, \cdot\right)\right) \\
& \leq h\left[K_{n, \theta, M, L}+K_{2} L \frac{|\mathfrak{a}(a, \delta)|}{\sigma} e^{-K_{2} p_{l}}+K_{2} L \frac{\mathfrak{a}(a, \delta)}{\sigma} e^{-K_{2}\left(1-p_{l}\right)}\right. \\
&\left.-K_{2}^{2} \frac{\mathfrak{a}(a, \delta)^{2}}{2} e^{-K_{2} p_{l}}-K_{2}^{2} \frac{\mathfrak{a}(a, \delta)^{2}}{2} e^{-K_{2}\left(1-p_{l}\right)}+\left|\varepsilon\left(h ; K_{2}\right)\right|\right] \\
& \leq h\left[\max _{h \in\left[0, h_{0}\right]}\left|\varepsilon\left(h ; K_{2}\right)\right|+K_{n, \theta, M, L}+K_{2}|\mathfrak{a}(a, \delta)|\left(e^{-K_{2} p_{l}}+e^{-K_{2}\left(1-p_{l}\right)}\right)\left(\frac{L}{\sigma}-\frac{|\mathfrak{a}(a, \delta)|}{2} K_{2}\right)\right] .
\end{aligned}
$$

To conclude, one can choose $K_{2}$ large enough so that

$$
K_{n, \theta, M, L}+K_{2}|\mathfrak{a}(a, \delta)|\left(e^{-K_{2} p_{l}}+e^{-K_{2}\left(1-p_{l}\right)}\right)\left(\frac{L}{\sigma}-\frac{\mid \mathfrak{a}(a, \delta)}{2} K_{2}\right) \leq-\eta<0,
$$

and then consider $h_{0}>0$ small enough so that $\left|\varepsilon\left(h ; K_{2}\right)\right| \leq \eta$ for $h \in\left[0, h_{0}\right]$.

Proposition 4.13 (monotonicity). Let $\pi$ be a grid of $[0, T]$ and $\delta>0$ satisfying (2.30)(2.32). Let $y \in \mathbb{R}, 0 \leq k \leq \kappa, j \in \mathbb{Z}, p \in[0,1]$, and let $\mathcal{U}, \mathcal{V}: \pi \times \delta \mathbb{Z} \times[0,1] \rightarrow \mathbb{R}$ be two bounded functions such that $\mathcal{U} \leq \mathcal{V}$. Then $\widehat{\mathfrak{S}}(\pi, \delta, j, k, p, y, \mathcal{U}) \geq \widehat{\mathfrak{S}}(\pi, \delta, j, k, p, y, \mathcal{V})$.

Proof. The result is clear for $j=\kappa$. If $j<\kappa$, it is sufficient to show that

$$
\mathcal{I}_{\delta}^{a}\left(S_{\delta}^{a}\left(t_{j}, t_{j+1}, \mathcal{U}\left(t_{j+1}, \cdot\right)\right)\right) \leq \mathcal{I}_{\delta}^{a}\left(S_{\delta}^{a}\left(t_{j}, t_{j+1}, \mathcal{V}\left(t_{j+1}, \cdot\right)\right)\right)
$$

for all $a \in K$, recalling the definition of $\widehat{\mathfrak{S}}$ in Definition 2.20. This is a consequence of the comparison result in Proposition 2.16 and the monotonicity of the linear interpolator.

We now prove the stability of the scheme. Here, in contrast to Lemma 4.5, we are not able to prove that the solution of the scheme is increasing in $p$. However, due to the boundedness of the terminal condition, we obtain uniform bounds for $v_{n, \pi, \delta}$.

Proposition 4.14 (stability). For all $\pi$ and $\delta>0$, there exists a unique solution $v_{n, \pi, \delta}$ to (2.37) which satisfies $0 \leq v_{n, \pi, \delta} \leq|g|_{\infty}$ on $\pi \times \delta \mathbb{Z} \times[0,1]$.

Proof. We prove the proposition by backward induction. First, since $v_{n, \pi, \delta}$ is a solution to $(2.37), v_{n, \pi, \delta}(T, x, p)=p g(x)$ on $\delta \mathbb{Z} \times[0,1]$, and we have $0 \leq v_{n, \pi, \delta}(T, x, p) \leq|g|_{\infty}$ for all $(x, p) \in \delta \mathbb{Z} \times[0,1]$.

Let $0 \leq j<\kappa$ and assume that $v_{n, \pi, \delta}\left(t_{j^{\prime}}, \cdot\right)$ is uniquely determined and satisfies $0 \leq$ $v_{n, \pi, \delta}\left(t_{j^{\prime}}, \cdot\right) \leq|g|_{\infty}$ for all $j^{\prime}>j$. Since $v_{n, \pi, \delta}$ is a solution to (2.37), we have

$$
v_{n, \pi, \delta}\left(t_{j}, x, p\right)=\min _{a \in K} \mathcal{I}_{\delta}^{a}\left(S_{\delta}^{a}\left(t_{j}, t_{j+1}, v_{n, \pi, \delta}\left(t_{j+1}, \cdot\right)\right)\right),
$$

and for each $a \in K, S_{\delta}^{a}\left(t_{j}, t_{j+1}, v_{n, \pi, \delta}\left(t_{j+1}, \cdot\right)\right)$ is uniquely determined by Proposition 2.15 , so $v_{n, \pi, \delta}\left(t_{j}, \cdot\right)$ is uniquely determined. Next, we show that

$$
0 \leq S_{\delta}^{a}\left(t_{j}, t_{j+1}, v_{n, \pi, \delta}\left(t_{j+1}, \cdot\right)\right) \leq|g|_{\infty} .
$$

Copyright (C) by SIAM. Unauthorized reproduction of this article is prohibited. 
Then it is easy to conclude that $0 \leq v_{n, \pi, \delta}\left(t_{j}, \cdot\right) \leq|g|_{\infty}$ on $\mathbb{R} \times[0,1]$, by properties of the linear interpolation and the minimization.

First, it is straightforward that $\check{u}$ defined by $\check{u}_{k, l}=0$ for all $k \in \mathbb{Z}$ and $0 \leq l \leq N_{a}$ satisfies $\check{u}=S_{\delta}^{a}\left(t_{j}, t_{j+1}, 0\right)$. The comparison theorem gives $0 \leq S_{\delta}^{a}\left(t_{j}, t_{j+1}, v_{n, \pi, \delta}\left(t_{j+1}, \cdot\right)\right)$, since $0 \leq v_{n, \pi, \delta}\left(t_{j+1}, \cdot\right)$. To obtain the upper bound, we notice that $\hat{u}$ defined by $\hat{u}_{k, l}:=|g|_{\infty}$ for all $k \in \mathbb{Z}$ and $0 \leq l \leq N_{a}$ satisfies

$$
S\left(k, l, \hat{u}_{k, l}, \nabla_{\delta}^{a} \hat{u}_{k, l}, \nabla_{+, \delta}^{a} \hat{u}_{k, l}, \Delta_{\delta}^{a} \hat{u}_{k, l}, \hat{u}\right)=-h f\left(t_{j}, x_{k},|g|_{\infty}, 0\right) \geq-h f\left(t_{j}, x_{k}, 0,0\right) \geq 0 .
$$

Hence the comparison result in Proposition 2.16 yields $S_{\delta}^{a}\left(t_{j}, t_{j+1}, v_{n, \pi, \delta}\left(t_{j+1}, \cdot\right)\right) \leq|g|_{\infty}$.

We now prove the consistency. The proof requires several lemmas. First, we state that the perturbation induced by the change of controls vanishes as $\delta \rightarrow 0$. The elementary proof is omitted.

Lemma 4.15. For all $a \in K, a$ and $\mathfrak{a}(a, \delta)$ have the same sign, and $0 \leq|a|-|\mathfrak{a}(a, \delta)| \leq \frac{n^{2}}{\sigma} \delta$. Moreover, there exists $c>0$ such that for all $a \in K$ and $\delta>0,|\mathfrak{a}(a, \delta)| \geq c>0$.

Last, we give explicit supersolutions and subsolutions satisfying appropriate conditions. Let $0 \leq t<s \leq T, \delta>0$ and $a \in K$ be fixed. For $\epsilon>0$, we set

$$
\begin{aligned}
f_{\epsilon}(t, x, y, \nu) & :=\left(f(t, \cdot, \cdot, \cdot) * \rho_{\epsilon}\right)(t, y, \nu) \\
& :=\int_{\mathbb{R} \times \mathbb{R} \times \mathbb{R}} f(t, x-u, y-z, \nu-\eta) \rho_{\epsilon}(u, z, \eta) \mathrm{d} u \mathrm{~d} z \mathrm{~d} \eta,
\end{aligned}
$$

where $*$ is the convolution operator and, for $\epsilon>0, \rho_{\epsilon}(x):=\epsilon^{-3} \rho(x / \epsilon)$ with $\rho: \mathbb{R}^{3} \rightarrow \mathbb{R}$ is a mollifier, i.e., a smooth function supported on $[-1,1]^{3}$ satisfying $\int_{\mathbb{R}} \rho=1$. We set

$$
F_{\epsilon}(t, x, y, q, A)=\left(\frac{1}{2} \sigma^{2}-\mu\right) q-\frac{\sigma^{2}}{2} A-f_{\epsilon}(t, x, y, \sigma q) .
$$

Remark 4.16. Since $f$ is L-Lipschitz-continuous with respect to its three last variables, we have $\left|f_{\epsilon}-f\right|_{\infty} \leq L \epsilon$.

Lemma 4.17. Let $0 \leq t<s \leq T, \varphi \in \mathcal{C}_{b}^{\infty}(\mathbb{R} \times \mathbb{R}, \mathbb{R}), a \in K$. We set $h:=s-t$. Let $\epsilon>0$ such that $\epsilon \rightarrow 0$ and $\frac{\delta}{\epsilon^{2}} \rightarrow 0$ as $h \rightarrow 0$, observing (2.32).

Then there exist bounded functions $S_{\delta}^{a, \pm}(t, s, \varphi): \delta \mathbb{Z} \times[0,1] \rightarrow \mathbb{R}$ of the form

$$
\begin{aligned}
S_{\delta, \epsilon}^{a, \pm}(t, s, \varphi)(x, p)= & \varphi\left(x, \mathfrak{p}^{a}(p)\right) \\
& -h F_{\epsilon}\left(t, x, \varphi\left(x, \mathfrak{p}^{a}(p)\right), \nabla^{\mathfrak{a}(a, \delta)} \varphi\left(x, \mathfrak{p}^{a}(p)\right), \Delta^{\mathfrak{a}(a, \delta)} \varphi\left(x, \mathfrak{p}^{a}(p)\right)\right) \\
& \pm C_{\varphi, n}(h, \epsilon),
\end{aligned}
$$

where $\mathfrak{p}^{a}$ is defined in (2.29), and where $C_{\varphi, n}(h, \epsilon)>0$ satisfies $\frac{C_{\varphi, n}(h, \epsilon)}{h} \rightarrow 0$ as $h \rightarrow 0$, such

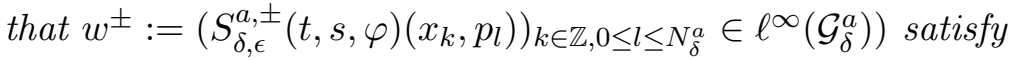

$$
\begin{aligned}
& S\left(k, l, w_{k, l}^{+}, \nabla_{\delta}^{a} w_{k, l}^{+}, \nabla_{+, \delta}^{a} w_{k, l}^{+}, \Delta_{\delta}^{a} w_{k, l}^{+}\right) \geq 0, \\
& S\left(k, l, w_{k, l}^{-}, \nabla_{\delta}^{a} w_{k, l}^{-}, \nabla_{+, \delta}^{a} w_{k, l}^{-}, \Delta_{\delta}^{a} w_{k, l}^{-}\right) \leq 0
\end{aligned}
$$

for all $k \in \mathbb{Z}$ and $0<l<N_{\delta}^{a}$.

Copyright (C) by SIAM. Unauthorized reproduction of this article is prohibited. 
Furthermore, for all $x \in \delta \mathbb{Z}, S_{\delta, \epsilon}^{a, \pm}(t, s, \varphi)(x, \cdot) \in \mathcal{C}^{2}([0,1], \mathbb{R})$, and $\left|\partial_{p p}^{2} S_{\delta, \epsilon}^{a, \pm}(t, s, \varphi)\right|_{\infty} \leq$ $\frac{C_{\varphi}(h)}{\epsilon^{2}}$ for some constant $C_{\varphi}(h)>0$ independent of $\epsilon$.

Proof. We show the result for $S_{\delta, \epsilon}^{a,+}$, while the proof is similar for $S_{\delta, \epsilon}^{a,-}$. For $k \in \mathbb{Z}$ and $0 \leq l \leq N_{\delta}^{a}$, let

$$
z_{k, l}:=\varphi\left(x_{k}, \mathfrak{p}^{a}\left(p_{l}\right)\right)-h F_{\epsilon}\left(t, x_{k}, \varphi\left(x, \mathfrak{p}^{a}\left(p_{l}\right)\right), \nabla^{\mathfrak{a}(a, \delta)} \varphi\left(x_{k}, \mathfrak{p}^{a}\left(p_{l}\right)\right), \Delta^{\mathfrak{a}(a, \delta)} \varphi\left(x_{k}, \mathfrak{p}^{a}\left(p_{l}\right)\right)\right) .
$$

It is enough to show that, for all $k \in \mathbb{Z}$ and $0<l<N_{\delta}^{a}$,

$$
S\left(k, l, z_{k, l}, \nabla_{\delta}^{a} z_{k, l}, \nabla_{+, \delta}^{a} z_{k, l}, \Delta_{\delta}^{a} z_{k, l}, \varphi\right) \geq-C_{\varphi, n}(h, \epsilon) .
$$

Then, since $f$ is nonincreasing in its third variable, it is then easy to show that $w^{+}=z+$ $C_{\varphi, n}(h, \epsilon)$ satisfies (4.16).

Let $k \in \mathbb{Z}$ and $1 \leq l \leq N_{a}-1$. We have, by definition (2.28),

$$
\begin{aligned}
& S\left(k, l, z_{k, l}, \nabla_{\delta}^{a} z_{k, l}, \nabla_{+, \delta}^{a} z_{k, l}, \Delta_{\delta}^{a} z_{k, l}, \varphi\right) \\
& =h\left(\widehat{F}\left(t, x_{k}, z_{k, l}, \nabla_{\delta}^{a} z_{k, l}, \nabla_{+, \delta}^{a} z_{k, l}, \Delta_{\delta}^{a} z_{k, l}\right)\right. \\
& \left.\quad-F_{\epsilon}\left(t, x_{k}, \varphi\left(x_{k}, \mathfrak{p}^{a}\left(p_{l}\right)\right), \nabla^{\mathfrak{a}(a, \delta)} \varphi\left(x_{k}, \mathfrak{p}^{a}\left(p_{l}\right)\right), \Delta^{\mathfrak{a}(a, \delta)} \varphi\left(x_{k}, \mathfrak{p}^{a}\left(p_{l}\right)\right)\right)\right),
\end{aligned}
$$

so it is enough to show

$$
\begin{aligned}
& \widehat{F}\left(t, x_{k}, z_{k, l}, \nabla_{\delta}^{a} z_{k, l}, \nabla_{+, \delta}^{a} z_{k, l}, \Delta_{\delta}^{a} z_{k, l}\right) \\
& \quad-F_{\epsilon}\left(t, x_{k}, \varphi\left(x_{k}, \mathfrak{p}^{a}\left(p_{l}\right)\right), \nabla^{\mathfrak{a}(a, \delta)} \varphi\left(x_{k}, \mathfrak{p}^{a}\left(p_{l}\right)\right), \Delta^{\mathfrak{a}(a, \delta)} \varphi\left(x_{k}, \mathfrak{p}^{a}\left(p_{l}\right)\right)\right) \geq-\frac{C_{\phi, n}(h, \epsilon)}{h} .
\end{aligned}
$$

We split the sum into three terms:

$$
\begin{aligned}
A= & \widehat{F}\left(t, x_{k}, z_{k, l}, \nabla_{\delta}^{a} z_{k, l}, \nabla_{+, \delta}^{a} z_{k, l}, \Delta_{\delta}^{a} z_{k, l}\right) \\
& -\widehat{F}\left(t, x_{k}, \varphi\left(x_{k}, \mathfrak{p}^{a}\left(p_{l}\right)\right), \nabla_{\delta}^{a} \varphi\left(x_{k}, \mathfrak{p}^{a}\left(p_{l}\right)\right), \nabla_{+, \delta}^{a} \varphi\left(x_{k}, \mathfrak{p}^{a}\left(p_{l}\right)\right), \Delta_{\delta}^{a} \varphi\left(x_{k}, \mathfrak{p}^{a}\left(p_{l}\right)\right)\right), \\
B= & \widehat{F}\left(t, x_{k}, \varphi\left(x_{k}, \mathfrak{p}^{a}\left(p_{l}\right)\right), \nabla_{\delta}^{a} \varphi\left(x_{k}, \mathfrak{p}^{a}\left(p_{l}\right)\right), \nabla_{+, \delta}^{a} \varphi\left(x_{k}, \mathfrak{p}^{a}\left(p_{l}\right)\right), \Delta_{\delta}^{a} \varphi\left(x_{k}, \mathfrak{p}^{a}\left(p_{l}\right)\right)\right) \\
& -F\left(t, x_{k}, \varphi\left(x_{k}, \mathfrak{p}^{a}\left(p_{l}\right)\right), \nabla^{\mathfrak{a}(a, \delta)} \varphi\left(x_{k}, \mathfrak{p}^{a}\left(p_{l}\right)\right), \Delta^{\mathfrak{a}(a, \delta)} \varphi\left(x_{k}, \mathfrak{p}^{a}\left(p_{l}\right)\right)\right), \\
C= & F\left(t, x_{k}, \varphi\left(x_{k}, \mathfrak{p}^{a}\left(p_{l}\right)\right), \nabla^{\mathfrak{a}(a, \delta)} \varphi\left(x_{k}, \mathfrak{p}^{a}\left(p_{l}\right)\right), \Delta^{\mathfrak{a}(a, \delta)} \varphi\left(x_{k}, \mathfrak{p}^{a}\left(p_{l}\right)\right)\right) \\
& -F_{\epsilon}\left(t, x_{k}, \varphi\left(x_{k}, \mathfrak{p}^{a}\left(p_{l}\right)\right), \nabla^{\mathfrak{a}(a, \delta)} \varphi\left(x_{k}, \mathfrak{p}^{a}\left(p_{l}\right)\right), \Delta^{\mathfrak{a}(a, \delta)} \varphi\left(x_{k}, \mathfrak{p}^{a}\left(p_{l}\right)\right)\right) .
\end{aligned}
$$

First, we have

$$
\begin{aligned}
C & =f_{\epsilon}\left(t, x_{k}, \varphi\left(x_{k}, \mathfrak{p}^{a}\left(p_{l}\right)\right), \nabla^{\mathfrak{a}(a, \delta)} \varphi\left(x_{k}, \mathfrak{p}^{a}\left(p_{l}\right)\right)\right)-f\left(t, x_{k}, \varphi\left(x_{k}, \mathfrak{p}^{a}\left(p_{l}\right)\right), \nabla^{\mathfrak{a}(a, \delta)} \varphi\left(x_{k}, \mathfrak{p}^{a}\left(p_{l}\right)\right)\right) \\
& \geq-\left|f_{\epsilon}-f\right|_{\infty} .
\end{aligned}
$$

Copyright (C by SIAM. Unauthorized reproduction of this article is prohibited. 
Second, by (2.22)-(2.23), we have

$$
\begin{aligned}
B & =-\theta \frac{\delta^{2}}{h} \Delta_{\delta}^{a} \varphi\left(x_{k}, \mathfrak{p}^{a}\left(p_{l}\right)\right) \\
& +\mu\left(\nabla^{\mathfrak{a}(a, \delta)} \varphi\left(x_{k}, \mathfrak{p}^{a}\left(p_{l}\right)\right)-\nabla_{+, \delta}^{a} \varphi\left(x_{k}, \mathfrak{p}^{a}\left(p_{l}\right)\right)\right)+\frac{\sigma^{2}}{2}\left(\Delta^{\mathfrak{a}(a, \delta)} \varphi\left(x_{k}, \mathfrak{p}^{a}\left(p_{l}\right)\right)-\Delta_{\delta}^{a} \varphi\left(x_{k}, \mathfrak{p}^{a}\left(p_{l}\right)\right)\right) \\
& +\left(f\left(t, x_{k}, \varphi\left(x_{k}, \mathfrak{p}^{a}\left(p_{l}\right)\right), \sigma \nabla^{\mathfrak{a}(a, \delta)} \varphi\left(x_{k}, \mathfrak{p}^{a}\left(p_{l}\right)\right)\right)-f\left(t, x_{k}, \varphi\left(x_{k}, \mathfrak{p}^{a}\left(p_{l}\right)\right), \sigma \nabla_{\delta}^{a} \varphi\left(x_{k}, \mathfrak{p}^{a}\left(p_{l}\right)\right)\right)\right) \\
& \geq-\theta \frac{\delta^{2}}{h}\left|\Delta_{\delta}^{a} \varphi\left(x_{k}, \mathfrak{p}^{a}\left(p_{l}\right)\right)\right| \\
& \left.-\mu \mid \nabla^{\mathfrak{a}(a, \delta)} \varphi\left(x_{k}, \mathfrak{p}^{a}\left(p_{l}\right)\right)-\nabla_{+, \delta}^{a} \varphi\left(x_{k}, \mathfrak{p}^{a}\left(p_{l}\right)\right)\right)-\frac{\sigma^{2}}{2}\left|\Delta^{\mathfrak{a}(a, \delta)} \varphi\left(x_{k}, \mathfrak{p}^{a}\left(p_{l}\right)\right)-\Delta_{\delta}^{a} \varphi\left(x_{k}, \mathfrak{p}^{a}\left(p_{l}\right)\right)\right| \\
& -\sigma L\left|\nabla^{\mathfrak{a}(a, \delta)} \varphi\left(x_{k}, \mathfrak{p}^{a}\left(p_{l}\right)\right)-\nabla_{\delta}^{a} \varphi\left(x_{k}, \mathfrak{p}^{a}\left(p_{l}\right)\right)\right| .
\end{aligned}
$$

The first term goes to 0 since $\frac{\delta^{2}}{h} \rightarrow 0$ as $h \rightarrow 0$ and $\Delta_{\delta}^{a} \varphi\left(x_{k}, \mathfrak{p}^{a}\left(p_{l}\right)\right)$ is bounded. The last three terms go to 0 by Taylor expansion and Lemma 4.15, since $\varphi$ is smooth.

Finally, by (2.22)-(2.23), using the linearity of the discrete differential operators and the definition of $z$, and since $f$ is Lipschitz-continuous, we have

$$
\begin{aligned}
A \geq & -h \mu\left|\nabla_{+, \delta}^{a} F_{\epsilon}\left(t, x_{k}, \varphi\left(x_{k}, \mathfrak{p}^{a}\left(p_{l}\right)\right), \nabla^{\mathfrak{a}(a, \delta)} \varphi\left(x_{k}, \mathfrak{p}^{a}\left(p_{l}\right)\right), \Delta^{\mathfrak{a}(a, \delta)} \varphi\left(x_{k}, \mathfrak{p}^{a}\left(p_{l}\right)\right)\right)\right| \\
& -h\left(\frac{\sigma^{2}}{2}+\theta \frac{\delta^{2}}{h}\right)\left|\Delta_{\delta}^{a} F_{\epsilon}\left(t, x_{k}, \varphi\left(x_{k}, \mathfrak{p}^{a}\left(p_{l}\right)\right), \nabla^{\mathfrak{a}(a, \delta)} \varphi\left(x_{k}, \mathfrak{p}^{a}\left(p_{l}\right)\right), \Delta^{\mathfrak{a}(a, \delta)} \varphi\left(x_{k}, \mathfrak{p}^{a}\left(p_{l}\right)\right)\right)\right| \\
& -L h\left|F_{\epsilon}\left(t, x_{k}, \varphi\left(x_{k}, \mathfrak{p}^{a}\left(p_{l}\right)\right), \nabla^{\mathfrak{a}(a, \delta)} \varphi\left(x_{k}, \mathfrak{p}^{a}\left(p_{l}\right)\right), \Delta^{\mathfrak{a}(a, \delta)} \varphi\left(x_{k}, \mathfrak{p}^{a}\left(p_{l}\right)\right)\right)\right| \\
& -L \sigma h\left|\nabla_{\delta}^{a} F_{\epsilon}\left(t, x_{k}, \varphi\left(x_{k}, \mathfrak{p}^{a}\left(p_{l}\right)\right), \nabla^{\mathfrak{a}(a, \delta)} \varphi\left(x_{k}, \mathfrak{p}^{a}\left(p_{l}\right)\right), \Delta^{\mathfrak{a}(a, \delta)} \varphi\left(x_{k}, \mathfrak{p}^{a}\left(p_{l}\right)\right)\right)\right| .
\end{aligned}
$$

We can show that each term goes to 0 as $h \rightarrow 0$. For example,

$$
\begin{aligned}
h \frac{\sigma^{2}}{2} \mid \Delta_{\delta}^{a} & F_{\epsilon}\left(t, x_{k}, \varphi\left(x_{k}, \mathfrak{p}^{a}\left(p_{l}\right)\right), \nabla^{\mathfrak{a}(a, \delta)} \varphi\left(x_{k}, \mathfrak{p}^{a}\left(p_{l}\right)\right), \Delta^{\mathfrak{a}(a, \delta)} \varphi\left(x_{k}, \mathfrak{p}^{a}\left(p_{l}\right)\right)\right) \mid \\
\geq & h \frac{\sigma^{2}}{2} \mu\left|\Delta_{\delta}^{a} \nabla^{\mathfrak{a}(a, \delta)} \varphi\left(x_{k}, \mathfrak{p}^{a}\left(p_{l}\right)\right)\right|-h \frac{\sigma^{4}}{4}\left|\Delta_{\delta}^{a} \Delta^{\mathfrak{a}(a, \delta)} \varphi\left(x_{k}, \mathfrak{p}^{a}\left(p_{l}\right)\right)\right| \\
& -h \frac{\sigma^{2}}{2}\left|\Delta_{\delta}^{a} f_{\epsilon}\left(t, x_{k}, \varphi\left(x_{k}, \mathfrak{p}^{a}\left(p_{l}\right)\right), \sigma \nabla_{\delta}^{a} \varphi\left(x_{k}, \mathfrak{p}^{a}\left(p_{l}\right)\right)\right)\right| .
\end{aligned}
$$

The first two terms go to 0 with $h$ since $\left|\Delta_{\delta}^{a} \nabla^{\mathfrak{a}(a, \delta)} \varphi\left(x_{k}, \mathfrak{p}^{a}\left(p_{l}\right)\right)\right|$ and $\left|\Delta_{\delta}^{a} \Delta^{\mathfrak{a}(a, \delta)} \varphi\left(x_{k}, \mathfrak{p}^{a}\left(p_{l}\right)\right)\right|$ are bounded, by the smoothness of $\varphi$, and by Lemma 4.15 .

We can control the derivatives of $\mathfrak{f}_{\epsilon}:(x, p) \mapsto f_{\epsilon}(t, x, \varphi(x, p), \sigma \varphi(x, p))$ with respect to $\epsilon$ : for any $\alpha=\left(\alpha_{1}, \alpha_{2}\right) \in \mathbb{N}^{2}$, we have

$$
\left|D^{\alpha} \mathfrak{f}_{\epsilon}\right|_{\infty} \leq \frac{C_{\varphi, \alpha}}{\epsilon^{\alpha_{1}+\alpha_{2}}}
$$

Copyright $\odot$ by SIAM. Unauthorized reproduction of this article is prohibited. 
for a constant $C_{\varphi, \alpha}>0$. By the triangle inequality and Taylor expansion, we get

$$
\begin{aligned}
-h \frac{\sigma^{2}}{2} & \left|\Delta_{\delta}^{a} f_{\epsilon}\left(t, x_{k}, \varphi\left(x_{k}, \mathfrak{p}^{a}\left(p_{l}\right)\right), \sigma \nabla_{\delta}^{a} \varphi\left(x_{k}, \mathfrak{p}^{a}\left(p_{l}\right)\right)\right)\right| \\
& \geq-h \frac{\sigma^{2}}{2}\left|\left(\Delta_{\delta}^{a}-\Delta^{\mathfrak{a}(a, \delta)}\right) f_{\epsilon}\left(t, x_{k}, \varphi\left(x_{k}, \mathfrak{p}^{a}\left(p_{l}\right)\right), \sigma \nabla_{\delta}^{a} \varphi\left(x_{k}, \mathfrak{p}^{a}\left(p_{l}\right)\right)\right)\right| \\
& -h \frac{\sigma^{2}}{2}\left|\Delta^{\mathfrak{a}(a, \delta)} f_{\epsilon}\left(t, x_{k}, \varphi\left(x_{k}, \mathfrak{p}^{a}\left(p_{l}\right)\right), \sigma \nabla_{\delta}^{a} \varphi\left(x_{k}, \mathfrak{p}^{a}\left(p_{l}\right)\right)\right)\right| \\
& \geq-C_{1} h \frac{\sigma^{2}}{2} \frac{\delta^{2}}{\epsilon^{4}}-C_{2} h \frac{\sigma^{2}}{2} \frac{1}{\epsilon^{2}},
\end{aligned}
$$

where $C_{1}, C_{2}>0$, and this quantity goes to 0 by our choice of $\epsilon$.

Last, the smoothness of $S_{\delta, \epsilon}^{a, \pm}$ is straightforward by (4.15) and the control on its second derivative with respect to $p$ is obtained by (4.18).

Lemma 4.18. Let $0 \leq t<s \leq T, \delta>0, a \in K, \varphi \in \mathcal{C}_{b}^{\infty}(\mathbb{R} \times \mathbb{R})$ be fixed. Let $h=s-t$, $k \in \mathbb{Z}, x_{k} \in \delta \mathbb{Z}, p \in(0,1)$, and assume that $h$ is sufficiently small so that $p \in\left[p_{1}, p_{N_{\delta}^{a}-1}\right]$, observing (2.32). Let $\epsilon>0$ such that $\epsilon \rightarrow 0$ and $\frac{\delta}{\epsilon^{2}} \rightarrow 0$ as $h \rightarrow 0$. Then we have

$$
\begin{aligned}
& S_{\delta, \epsilon}^{a,-}(t, s, \varphi)\left(x_{k}, p\right)-\mathcal{I}_{\delta}^{a}\left(S_{\delta}^{a}(t, s, \varphi)\right)\left(x_{k}, p\right) \leq C_{\varphi, n}^{\prime}(h, \epsilon), \\
& \mathcal{I}_{\delta}^{a}\left(S_{\delta}^{a}(t, s, \varphi)\right)\left(x_{k}, p\right)-S_{\delta, \epsilon}^{a,+}(t, s, \varphi)\left(x_{k}, p\right) \leq C_{\varphi, n}^{\prime}(h, \epsilon),
\end{aligned}
$$

where $C_{\varphi, n}^{\prime}(h, \epsilon)>0$ satisfies $\frac{C_{\varphi, n}^{\prime}(h, \epsilon)}{h} \rightarrow 0$ as $h \rightarrow 0$ and where the functions $S_{\delta, \epsilon}^{a, \pm}(s, t, \varphi)$ are introduced in Lemma 4.17.

Proof. We prove the first identity, while the proof of the second one is similar. Set $w:=$ $S_{\delta}^{a}(t, s, \varphi)$ and $w^{-}:=S_{\delta, \epsilon}^{a,-}(t, s, \varphi)$. By the definition of $w$ and by (4.17), one can apply Proposition 2.17. For all $k \in \mathbb{Z}$ and $0<l<N_{\delta}^{a}$,

$$
w_{k, l}^{-}-w_{k, l} \leq B e^{-4 \frac{\mathfrak{a}(a, \delta)^{2}}{\sigma^{2}} C(h, \delta) l\left(N_{\delta}^{a}-l\right)} \leq B e^{-4 \frac{\mathfrak{a}(a, \delta)^{2}}{\sigma^{2}} C(h, \delta)\left(N_{\delta}^{a}-1\right)}
$$

with $B=\left|\left(w_{\cdot, 0}^{-}-w_{\cdot, 0}\right)^{+}\right|_{\infty}+\left|\left(w_{\cdot, N_{\delta}^{a}}^{-}-w_{\cdot, N_{\delta}^{a}}\right)^{+}\right|_{\infty}$ and $C(h, \delta)$ is defined in (2.35). By Lemma 4.15 , there exists a constant $c>0$ such that $|\mathfrak{a}(a, \delta)| \geq c$. In addition, using (2.36), we get

$$
\begin{aligned}
\frac{B}{h} e^{-4 C(h, \delta) \frac{\mathfrak{a}(a, \delta)^{2}}{\sigma}\left(N_{\delta}^{a}-1\right)} & \leq \frac{B}{h} e^{-4 \frac{c^{2}}{\sigma^{2}}\left(\frac{1}{\left(\left(\mu+\frac{L}{2}\right) M+2 \theta M^{2}\right) h^{2}+\sigma^{2} h}-\frac{M^{2}}{2 \sigma^{4}}\right)} \\
& =B e^{4 \frac{c^{2} M^{2}}{2 \sigma^{6}}} \frac{e^{-4 \frac{c^{2}}{\sigma^{2}} \frac{1}{\left(\left(\mu+\frac{L}{2}\right) M+2 \theta M^{2}\right) h^{2}+\sigma^{2} h}}}{h} \rightarrow 0 \quad \text { as } h \rightarrow 0 .
\end{aligned}
$$

Now let $p \in\left[p_{1}, p_{N_{\delta}^{a}-1}\right)$ and $k \in \mathbb{Z}$. By the definition of $\mathcal{I}_{\delta}^{a}$, one has

$$
\mathcal{I}_{\delta}^{a}(w)\left(x_{k}, p\right)=\lambda w_{k, l}+(1-\lambda) w_{k, l+1},
$$

Copyright (C) by SIAM. Unauthorized reproduction of this article is prohibited. 
where $p \in\left[p_{l}, p_{l+1}\right)$ with $0<l<N_{\delta}^{a}-1$ and $\lambda=\frac{p_{l+1}-p}{p_{l+1}-p_{l}}$. Thus

$$
\begin{aligned}
& S_{\delta, \epsilon}^{a,-}(t, s, \varphi)\left(x_{k}, p\right)-\mathcal{I}_{\delta}^{a}(w)\left(x_{k}, p\right) \\
& =S_{\delta, \epsilon}^{a,-}(t, s, \varphi)\left(x_{k}, p\right)-\mathcal{I}_{\delta}^{a}\left(w^{-}\right)\left(x_{k}, p\right)+\mathcal{I}_{\delta}^{a}\left(w^{-}\right)\left(x_{k}, p\right)-\mathcal{I}_{\delta}^{a}(w)\left(x_{k}, p\right) \\
& =S_{\delta, \epsilon}^{a,-}(t, s, \varphi)\left(x_{k}, p\right)-\mathcal{I}_{\delta}^{a}\left(w^{-}\right)\left(x_{k}, p\right)+\lambda\left(w_{k, l}^{-}-w_{k, l}\right)+(1-\lambda)\left(w_{k, l+1}^{-}-w_{k, l+1}\right) .
\end{aligned}
$$

The two last terms are controlled using (4.19), and, by properties of the linear interpolation of the function $p \mapsto S_{\delta, \epsilon}^{a,-}(t, s, \varphi)\left(x_{k}, p\right) \in \mathcal{C}^{2}([0,1], \mathbb{R})$ with $\left|\partial_{p p}^{2} S_{\delta, \epsilon}^{a,-}(t, s, \varphi)\right|_{\infty} \leq \frac{C_{\varphi}(h)}{\epsilon^{2}}$ (recall the previous lemma) the first term is of order $\frac{\delta^{2}}{\epsilon^{2}}=o(h)$ since $(2.32)$ is in force and $\frac{\delta}{\epsilon^{2}} \rightarrow 0$.

Lemma 4.19. For $0 \leq t<s \leq T$ such that $L(s-t) \leq 1, \xi>0, \varphi: \delta \mathbb{Z} \times[0,1] \rightarrow \mathbb{R} a$ bounded function, the following holds for all $a \in K$ :

$$
S_{\delta}^{a}(t, s, \varphi)+\xi-L(s-t) \xi \leq S_{\delta}^{a}(t, s, \varphi+\xi) \leq S_{\delta}^{a}(t, s, \varphi)+\xi,
$$

where $L$ is the Lipschitz constant of $f$.

Proof. Let $v=S_{\delta}^{a}(t, s, \varphi), w=v+\xi-L(s-t) \xi$. Since $v$ satisfies (2.26), we have, for $k \in \mathbb{Z}$ and $0<l<N_{\delta}^{a}$,

$$
\begin{aligned}
& S\left(k, l, w_{k, l}, \nabla_{\delta}^{a} w_{k, l}, \nabla_{+, \delta}^{a} w_{k, l}, \Delta_{\delta}^{a} w_{k, l}, \varphi+\xi\right)=-L(s-t) \xi \\
& +(s-t)\left(f\left(t, x_{k}, v_{k, l}, \nabla_{\delta}^{a} v_{k, l}\right)-f\left(t, x_{k}, w_{k, l}, \nabla_{\delta}^{a} v_{k, l}\right)\right) .
\end{aligned}
$$

Since $f$ is nonincreasing in its third variable and Lipschitz-continuous, we get

$$
S\left(k, l, w_{k, l}, \nabla_{\delta}^{a} w_{k, l}, \nabla_{+, \delta}^{a} w_{k, l}, \Delta_{\delta}^{a} w_{k, l}, \varphi+\xi\right) \leq 0 .
$$

The same computation with $l=0$ or $l=N_{\delta}^{a}$ and $S_{b}$ instead of $S$ gives

$$
S_{b}\left(k, l, w_{k, l}, \nabla_{\delta} w_{k, l}, \nabla_{+, \delta} w_{k, l}, \Delta_{\delta} w_{k, l}, \varphi+\xi\right) \leq 0,
$$

and the comparison theorem given in Proposition 4.10 gives $w_{k, l} \leq S_{\delta}^{a}(t, s, \varphi+\xi)_{k, l}$ for $k \in \mathbb{Z}$ and $l \in\left\{0, N_{\delta}^{a}\right\}$.

The comparison result from Proposition 2.16 gives the first inequality of the lemma. The second one is proved similarly.

Proposition 4.20 (consistency). Let $\varphi \in \mathcal{C}_{b}^{\infty}([0, T] \times \mathbb{R} \times \mathbb{R}, \mathbb{R}),(t, x, p) \in[0, T) \times \mathbb{R} \times(0,1)$. We have, with the notation in (2.19),

$$
\begin{aligned}
& \mid \frac{1}{t_{j+1}-t_{j}} \widehat{\mathfrak{S}}\left(\pi, \delta, j, k, q, \varphi\left(t_{j}, x_{k}, q\right)+\xi, \varphi+\xi\right) \\
& \quad-\sup _{a \in K}\left[-D^{a} \varphi(t, x, p)+F\left(t, x, \varphi(t, x, p), \nabla^{a} \varphi(t, x, p), \Delta^{a} \varphi(t, x, p)\right)\right] \mid \rightarrow 0
\end{aligned}
$$

as $\delta,|\pi| \rightarrow 0$ with $h=t_{j+1}-t_{j}$ and $\delta$ satisfying (2.30)-(2.32) for all $0 \leq j<\kappa, \pi \times \delta \mathbb{Z} \times[0,1] \ni$ $\left(t_{j}, x_{k}, q\right) \rightarrow(t, x, p)$, and $\xi \rightarrow 0$. 
Proof. Let $\varphi, j, k, p, l$ be as in the statement of the Proposition. Without loss of generality, we can consider $\pi, \delta, t_{j}, x_{k}, q$ such that, for all $a \in K$,

$$
0 \leq \mathfrak{p}^{a}(q) \leq 1
$$

Since $\varphi$ is smooth and $\left(t_{k}, x_{j}, p_{l}\right) \rightarrow(t, x, p)$, we have

$$
\begin{aligned}
& \mid \sup _{a \in K}\left[-D^{a} \varphi(t, x, p)+F\left(t, x, p, \varphi(t, x, p), \nabla^{a} \varphi(t, x, p), \Delta^{a} \varphi(t, x, p)\right)\right] \\
& \left.\quad-\sup _{a \in K}\left[-D^{a} \varphi\left(t_{j}, x_{k}, p_{l}\right)+F\left(t_{j}, x_{k}, p_{l}, \varphi\left(t_{j}, x_{k}, p_{l}\right), \nabla^{a} \varphi\left(t_{j}, x_{k}, p_{l}\right), \Delta^{a} \varphi\left(t_{j}, x_{k}, p_{l}\right)\right)\right)\right] \mid \rightarrow 0 .
\end{aligned}
$$

Thanks to Lemma 4.19, it suffices to prove

$$
\begin{aligned}
& \mid \frac{1}{t_{j+1}-t_{j}} \widehat{\mathfrak{S}}\left(\pi, \delta, j, k, q, \varphi\left(t_{j}, x_{k}, q\right), \varphi\right) \\
& \quad-\sup _{a \in K}\left(-D^{a} \varphi\left(t_{j}, x_{k}, q\right)+F\left(t_{j}, x_{k}, \varphi\left(t_{j}, x_{k}, q\right), \nabla^{a} \varphi\left(t_{j}, x_{k}, q\right), \Delta^{a} \varphi\left(t_{j}, x_{k}, q\right)\right)\right) \mid \rightarrow 0
\end{aligned}
$$

as $|\pi| \rightarrow 0$ and $\pi \times \delta \mathbb{Z} \times(0,1) \ni\left(t_{j}, x_{k}, q\right) \rightarrow(t, x, p)$.

Let $\epsilon>0$ such that $\epsilon \rightarrow 0$ and $\frac{\delta}{\epsilon^{2}} \rightarrow 0$. Using $|\inf -\sup | \leq \sup |\cdot-\cdot|$, adding

$$
\begin{aligned}
\pm & \left(\frac{1}{t_{j+1}-t_{j}} \varphi\left(t_{j+1}, x_{k}, \mathfrak{p}^{a}(q)\right)\right. \\
& \left.+F_{\epsilon}\left(t_{j}, x_{k}, \varphi\left(t_{j+1}, x_{k}, \mathfrak{p}^{a}(q)\right), \nabla^{\mathfrak{a}(a, \delta)} \varphi\left(t_{j+1}, x_{k}, \mathfrak{p}^{a}(q)\right), \Delta^{\mathfrak{a}(a, \delta)} \varphi\left(t_{j+1}, x_{k}, \mathfrak{p}^{a}(q)\right)\right)\right)
\end{aligned}
$$

and using Lemma 4.15, it is enough to show that, for all $a \in K$,

$$
\begin{aligned}
& \mid \frac{1}{t_{j+1}-t_{j}}\left(\varphi\left(t_{j+1}, x_{k}, \mathfrak{p}^{a}(q)\right)-\mathcal{I}_{\delta}^{a}\left(S_{\delta}^{a}\left(t_{j+1}, t_{j}, \varphi\left(t_{j+1}, \cdot\right)\right)\right)\left(x_{k}, q\right)\right) \\
& -F_{\epsilon}\left(t_{j}, x_{k}, \varphi\left(t_{j+1}, x_{k}, \mathfrak{p}^{a}(q)\right), \nabla^{\mathfrak{a}(a, \delta)} \varphi\left(t_{j+1}, x_{k}, \mathfrak{p}^{a}(q)\right), \Delta^{\mathfrak{a}(a, \delta)} \varphi\left(t_{j+1}, x_{k}, \mathfrak{p}^{a}(q)\right)\right) \mid \rightarrow 0 .
\end{aligned}
$$

The proof is concluded using the equality $|\cdot|=\max (\cdot,-\cdot)$ and the two following inequalities, obtained by Lemma 4.18 and by definition (4.16) to (4.17) of $S_{\delta, \epsilon}^{a, \pm}$ :

$$
\begin{aligned}
& \frac{1}{t_{j+1}-t_{j}}\left(\varphi\left(t_{j+1}, x_{k}, \mathfrak{p}^{a}(q)\right)-\mathcal{I}_{\delta}^{a}\left(S_{\delta}^{a}\left(t_{j+1}, t_{j}, \varphi\left(t_{j+1}, \cdot\right)\right)\right)\left(x_{k}, q\right)\right) \\
& -F_{\epsilon}\left(t_{j}, x_{k}, \varphi\left(t_{j+1}, x_{k}, \mathfrak{p}^{a}(q)\right), \nabla^{\mathfrak{a}(a, \delta)} \varphi\left(t_{j+1}, x_{k}, \mathfrak{p}^{a}(q)\right), \Delta^{\mathfrak{a}(a, \delta)} \varphi\left(t_{j+1}, x_{k}, \mathfrak{p}^{a}(q)\right)\right) \\
& \left.\leq \frac{1}{t_{j+1}-t_{j}}\left(\varphi\left(t_{j+1}, x_{k}, \mathfrak{p}^{a}(q)\right)-S_{\delta, \epsilon}^{a,-}\left(t_{j+1}, t_{j}, \varphi\left(t_{j+1}, \cdot\right)\right)\right)\left(x_{k}, q\right)+o\left(t_{j+1}-t_{j}\right)\right) \\
& -F_{\epsilon}\left(t_{j}, x_{k}, \varphi\left(t_{j+1}, x_{k}, \mathfrak{p}^{a}(q)\right), \nabla^{\mathfrak{a}(a, \delta)} \varphi\left(t_{j+1}, x_{k}, \mathfrak{p}^{a}(q)\right), \Delta^{\mathfrak{a}(a, \delta)} \varphi\left(t_{j+1}, x_{k}, \mathfrak{p}^{a}(q)\right)\right)
\end{aligned}
$$

Copyright (C) by SIAM. Unauthorized reproduction of this article is prohibited. 
and

$$
\begin{aligned}
& F_{\epsilon}\left(t_{j}, x_{k}, \varphi\left(t_{j+1}, x_{k}, \mathfrak{p}^{a}(q)\right), \nabla^{\mathfrak{a}(a, \delta)} \varphi\left(t_{j+1}, x_{k}, \mathfrak{p}^{a}(q)\right), \Delta^{\mathfrak{a}(a, \delta)} \varphi\left(t_{j+1}, x_{k}, \mathfrak{p}^{a}(q)\right)\right) \\
& -\frac{1}{t_{j+1}-t_{j}}\left(\varphi\left(t_{j+1}, x_{k}, \mathfrak{p}^{a}(q)\right)-\mathcal{I}_{\delta}^{a}\left(S_{\delta}^{a}\left(t_{j+1}, t_{j}, \varphi\left(t_{j+1}, \cdot\right)\right)\right)\left(x_{k}, q\right)\right) \\
& \leq F_{\epsilon}\left(t_{j}, x_{k}, \varphi\left(t_{j+1}, x_{k}, \mathfrak{p}^{a}(q)\right), \nabla^{\mathfrak{a}(a, \delta)} \varphi\left(t_{j+1}, x_{k}, \mathfrak{p}^{a}(q)\right), \Delta^{\mathfrak{a}(a, \delta)} \varphi\left(t_{j+1}, x_{k}, \mathfrak{p}^{a}(q)\right)\right) \\
& -\frac{1}{t_{j+1}-t_{j}}\left(\varphi\left(t_{j+1}, x_{k}, \mathfrak{p}^{a}(q)\right)-S_{\delta, \epsilon}^{a,+}\left(t_{j+1}, t_{j}, \varphi\left(t_{j+1}, \cdot\right)\right)\left(x_{k}, q\right)+o\left(t_{j+1}-t_{j}\right)\right) .
\end{aligned}
$$

4.5. Proof of Theorem 2.28. Let $e(j)=\max _{k \in \mathbb{Z}, p \in[0,1]}\left|v_{n, \pi, \delta}^{\mathrm{up}}\left(t_{\kappa-j}, x_{k}, p\right)-v_{n, \pi, \delta}^{\mathrm{CS}}\left(t_{\kappa-j}, x_{k}, p\right)\right|$ for all $0 \leq j \leq \kappa$. If we prove that $e(j) \leq C^{\prime} j \delta$, the theorem follows as $j \leq \kappa=\frac{T}{h}$ and $\frac{\delta}{h} \rightarrow 0$ as $h \rightarrow 0$.

We have $e(0)=0$ since $v_{n, \pi, \delta}^{\text {up }}(T, x, p)=v_{n, \pi, \delta}^{\mathrm{CS}}(T, x, p)=p g(x)$ on $\delta \mathbb{Z} \times[0,1]$. Assume that $e(j-1) \leq C^{\prime}(j-1) \delta$ for some $1 \leq j \leq \kappa$, and we are going to prove that $e(j) \leq C^{\prime} j \delta$. Let $k \in \mathbb{Z}$ and $p \in\left[p_{l}, p_{l+1}\right)$ for $0 \leq l<N_{\delta}^{a}$. We have

$$
\begin{aligned}
\left|v_{n, \pi, \delta}^{\mathrm{up}}\left(t_{\kappa-j+1}, x_{k}, p\right)-v_{n, \pi, \delta}^{\mathrm{CS}}\left(t_{\kappa-j+1}, x_{k}, p\right)\right| & =\left|\min _{a \in K} \mathcal{I}_{\delta}^{a}\left(w_{j}^{a}\right)\left(x_{k}, p\right)-\min _{a \in K} \mathcal{I}_{\delta}^{\mathrm{CS}, a}\left(w_{j}^{\mathrm{CS}, a}\right)\left(x_{k}, p\right)\right| \\
& \leq \sup _{a \in K}\left|\mathcal{I}_{\delta}^{a}\left(w_{j}^{a}\right)\left(x_{k}, p\right)-\mathcal{I}_{\delta}^{\mathrm{CS}, a}\left(w_{j}^{\mathrm{CS}, \mathrm{a}}\right)\left(x_{k}, p\right)\right| .
\end{aligned}
$$

By the induction hypothesis, $\left|v_{n, \pi, \delta}^{\mathrm{up}}\left(t_{\kappa-j+1}, \cdot\right)-v_{n, \pi, \delta}^{\mathrm{CS}}\left(t_{\kappa-j+1}, \cdot\right)\right| \leq C^{\prime}(j-1) \delta$ and using Lemma 4.19, we obtain

$$
\begin{aligned}
w_{j}^{a}-w_{j}^{\mathrm{CS}, \mathrm{a}} & =S_{\delta}^{a}\left(t_{\kappa-j}, t_{\kappa-j+1}, v_{n, \pi, \delta}^{\mathrm{up}}\left(t_{\kappa-j+1}, \cdot\right)\right)-S_{\delta}^{a}\left(t_{\kappa-j}, t_{\kappa-j+1}, v_{n, \pi, \delta}^{\mathrm{CS}}\left(t_{\kappa-j+1}, \cdot\right)\right) \\
& \leq S_{\delta}^{a}\left(t_{\kappa-j}, t_{\kappa-j+1}, v_{n, \pi, \delta}^{\mathrm{CS}}\left(t_{\kappa-j+1}, \cdot\right)\right)+C^{\prime}(j-1) \delta-S_{\delta}^{a}\left(t_{\kappa-j}, t_{\kappa-j+1}, v_{n, \pi, \delta}^{\mathrm{CS}}\left(t_{\kappa-j+1}, \cdot\right)\right) \\
& =C^{\prime}(j-1) \delta \text { and, similarly, } \\
w_{j}^{\mathrm{CS}, \mathrm{a}}-w_{j}^{a} & \leq C^{\prime}(j-1) \delta .
\end{aligned}
$$

To complete the induction, it therefore suffices to show that

$$
\left|\mathcal{I}_{\delta}^{a}\left(w_{j}^{a}\right)\left(x_{k}, p\right)-\mathcal{I}_{\delta}^{\mathrm{CS}, a}\left(w_{j}^{\mathrm{CS}, \mathrm{a}}\right)\left(x_{k}, p\right)\right| \leq\left|w_{j}^{a}-w_{j}^{\mathrm{CS}, a}\right|_{\infty}+\delta C^{\prime}
$$

for some $C^{\prime}$ independent of $a, \delta$, and $h$.

We first show this assuming (2.39). Then, for given $a$, we obtain directly

$$
\left|\mathcal{I}_{\delta}^{a}\left(w_{j}^{a}\right)\left(x_{k}, p\right)-\mathcal{I}_{\delta}^{\mathrm{CS}, a}\left(w_{j}^{\mathrm{CS}, a}\right)\left(x_{k}, p\right)\right| \leq\left|w_{j}^{a}-w_{j}^{\mathrm{CS}, a}\right|_{\infty}+\delta\left[v_{n, \pi, \delta}^{\mathrm{up}}\right]_{1},
$$

where we have used the fact that, by construction, the cubic spline interpolant is bounded in each interval by the two adjacent nodal values. Then (4.20) follows immediately with $C^{\prime}=C$.

Copyright (C) by SIAM. Unauthorized reproduction of this article is prohibited. 
Now assume instead that (2.40) holds. Fix again $a \in K$. We have, by the induction hypothesis as above,

$$
\begin{aligned}
& \left|\mathcal{I}_{\delta}^{a}\left(w_{j}^{a}\right)\left(x_{k}, p\right)-\mathcal{I}_{\delta}^{\mathrm{CS}, a}\left(w_{j}^{\mathrm{CS}, a}\right)\left(x_{k}, p\right)\right| \\
& \leq\left|\mathcal{I}_{\delta}^{a}\left(w_{j}^{a}\right)\left(x_{k}, p\right)-\mathcal{I}_{\delta}^{a}\left(w_{j}^{\mathrm{CS}, \mathrm{a}}\right)\left(x_{k}, p\right)\right|+\left|\mathcal{I}_{\delta}^{a}\left(w_{j}^{\mathrm{CS}, \mathrm{a}}\right)\left(x_{k}, p\right)-\mathcal{I}_{\delta}^{\mathrm{CS}, a}\left(w_{j}^{\mathrm{CS}, a}\right)\left(x_{k}, p\right)\right| \\
& \leq\left|w_{j}^{a}-w_{j}^{\mathrm{CS}, \mathrm{a}}\right| \infty+\left|\mathcal{I}_{\delta}^{a}\left(w_{j}^{\mathrm{CS}, \mathrm{a}}\right)\left(x_{k}, p\right)-\mathcal{I}_{\delta}^{\mathrm{CS}, \mathrm{a}}\left(w_{j}^{\mathrm{CS}, \mathrm{a}}\right)\left(x_{k}, p\right)\right| .
\end{aligned}
$$

Since

$$
\begin{aligned}
\mathcal{I}_{\delta}^{a}\left(w_{j}^{\mathrm{CS}, a}\right)\left(x_{k}, p\right)= & w_{j, k, l}^{\mathrm{CS}, \mathrm{a}} h_{0,0}\left(t_{\delta}^{a}(p)\right)+\frac{1}{N_{\delta}^{a}} \frac{w_{j, k, l+1}^{\mathrm{CS}, a}-w_{j, k, l}^{\mathrm{CS}, a}}{p_{l+1}-p_{l}} h_{1,0}\left(t_{\delta}^{a}(p)\right) \\
& +w_{j, k, l+1}^{\mathrm{CS}, a} h_{0,1}\left(t_{\delta}^{a}(p)\right)+\frac{1}{N_{\delta}^{a}} \frac{w_{j, k, l+1}^{\mathrm{CS}, a}-w_{j, k, l}^{\mathrm{CS}, a}}{p_{l+1}-p_{l}} h_{1,1}\left(t_{\delta}^{a}(p)\right) \text { and } \\
\mathcal{I}_{\delta}^{\mathrm{CS}, a}\left(w_{j}^{\mathrm{CS}, a}\right)\left(x_{k}, p\right)= & w_{j, k, l}^{\mathrm{CS}, a} h_{0,0}\left(t_{\delta}^{a}(p)\right)+\frac{1}{N_{\delta}^{a}} m_{\delta}^{a}\left(k, l, w_{j}^{\mathrm{CS}, a}\right) h_{1,0}\left(t_{\delta}^{a}(p)\right) \\
& +w_{j, k, l+1}^{\mathrm{CS}, a} h_{0,1}\left(t_{\delta}^{a}(p)\right)+\frac{1}{N_{\delta}^{a}} m_{\delta}^{a}\left(k, l+1, w_{j}^{\mathrm{CS}, a}\right) h_{1,1}\left(t_{\delta}^{a}(p)\right),
\end{aligned}
$$

we obtain from $\frac{1}{N_{\delta}^{a}} \leq \frac{|a| \delta}{\sigma} \leq \frac{\max _{a \in K}|a|}{\sigma} \delta$ and assumption (2.40) that

$$
\begin{aligned}
\left|\left(\mathcal{I}_{\delta}^{a}-\mathcal{I}_{\delta}^{\mathrm{CS}, a}\right)\left(w_{j}^{a}\right)\left(x_{k}, p\right)\right| \leq & \frac{1}{N_{\delta}^{a}}\left(\left|\frac{w_{j, k, l+1}^{a}-w_{j, k, l}^{a}}{p_{l+1}-p_{l}}-m_{\delta}^{a}\left(k, l, w_{j}^{\mathrm{CS}, \mathrm{a}}\right)\right|\left|h_{1,0}\right|_{\infty}\right. \\
& \left.+\left|\frac{w_{j, k, l+1}^{a}-w_{j, k, l}^{a}}{p_{l+1}-p_{l}}-m_{\delta}^{a}\left(k, l+1, w_{j}^{\mathrm{CS}, \mathrm{a}}\right)\right|\left|h_{1,1}\right|_{\infty}\right) \\
& \leq C^{\prime} \delta
\end{aligned}
$$

with $C^{\prime}=C \frac{\max _{a \in K} \mid a}{\sigma}\left(\left|h_{1,0}\right|_{\infty}+\left|h_{1,1}\right|_{\infty}\right)$.

Finally, we obtain $\left|\mathcal{I}_{\delta}^{a}\left(w_{j}^{a}\right)\left(x_{k}, p\right)-\mathcal{I}_{\delta}^{\mathrm{CS}, a}\left(w_{j}^{\mathrm{CS}, a}\right)\left(x_{k}, p\right)\right| \leq C^{\prime}(j-1) \delta+C^{\prime} \delta=C^{\prime} j \delta$. This completes the proof.

\section{REFERENCES}

[1] Y. Achdou, G. Barles, H. Ishiı, And G. L. Litvinov, Hamilton-Jacobi equations: Approximations, numerical analysis and applications, Lecture Notes in Math. 2074, Springer, Berlin, 2013.

[2] G. Barles, C. Daher, And M. Romano, Convergence of numerical schemes for parabolic equations arising in finance theory, Math. Models Methods Appl. Sci., 5 (1995), pp. 125-143.

[3] G. BARles AND E. R. Jakobsen, Error bounds for monotone approximation schemes for parabolic Hamilton-Jacobi-Bellman equations, Math. Comp., 76 (2007), pp. 1861-1893.

[4] G. Barles And P. E. Souganidis, Convergence of approximation schemes for fully nonlinear second order equations, Asymptot. Anal., 4 (1991), pp. 271-283.

[5] O. Bokanowski, B. Bruder, S. Maroso, and H. Zidani, Numerical approximation for a superreplication problem under gamma constraints, SIAM J. Numer. Anal., 47 (2009), pp. 2289-2320.

Copyright $\odot$ by SIAM. Unauthorized reproduction of this article is prohibited. 
[6] O. Bokanowski, A. Picarelli, and C. Reisinger, High-order filtered schemes for time-dependent second order HJB equations, ESAIM Math. Model. Numer. Anal., 52 (2018), pp. 69-97.

[7] J. F. Bonnans AND H. ZIDAnI, Consistency of generalized finite difference schemes for the stochastic HJB equation, SIAM J. Numer. Anal., 41 (2003), pp. 1008-1021.

[8] B. Bouchard, G. Bouveret, And J.-F. Chassagneux, A backward dual representation for the quantile hedging of Bermudan options, SIAM J. Financial Math., 7 (2016), pp. 215-235.

[9] B. Bouchard, R. Elie, and A. RÉveillac, BSDEs with weak terminal condition, Ann. Probab., 43 (2015), pp. 572-604.

[10] B. Bouchard, R. Elie, And N. TouzI, Stochastic target problems with controlled loss, SIAM J. Control Optim., 48 (2009), pp. 3123-3150.

[11] B. Bouchard And T. NAM VU, A stochastic target approach for PESL matching problems, Math. Oper. Res., 37 (2012), pp. 526-558.

[12] G. Bouveret and J.-F. Chassagneux, A comparison principle for PDEs arising in approximate hedging problems: Application to Bermudan options, Appl. Math. Optim., 78 (2018), pp. 469-491.

[13] J.-F. Chassagneux and A. Richou, Obliquely Reflected BSDEs, preprint, arXiv:1710.08989, 2018.

[14] M. G. Crandall, H. Ishit, And P.-L. Lions, Users guide to viscosity solutions of second order partial differential equations, Bull. Amer. Math. Soc. (N.S.), 43 (1992), pp. 1-19.

[15] M. G. Crandall And P.-L. Lions, Two approximations of solutions of Hamilton-Jacobi equations, Math. Comp., 43 (1984), pp. 1-19.

[16] R. W. DARling AND E. PARdoux, Backwards SDE with random terminal time and applications to semilinear elliptic PDE, Ann. Probab., 25 (1997), pp. 1135-1159.

[17] H. Dong AND N. KRYlov, The rate of convergence of finite-difference approximations for parabolic Bellman equations with Lipschitz coefficients in cylindrical domains, Appl. Math. Optim., 56 (2007), pp. 37-66.

[18] R. Dumitrescu, BSDEs with Nonlinear Weak Terminal Condition, preprint, https://arxiv.org/abs/1602. $00321,2016$.

[19] R. Dumitrescu, R. Elie, W. Sabbagh, And C. Zhou, BSDEs with Weak Reflections and Partial Hedging of American Options, preprint, https://arxiv.org/abs/1708.05957, 2017.

[20] R. Dumitrescu, C. Reisinger, and Y. Zhang, Approximation schemes for mixed optimal stopping and control problems with nonlinear expectations and jumps, Appl. Math. Optim., https://doi.org/ 10.1007/s00245-019-09591-0 (2019).

[21] N. El Karoui, S. Peng, And M.-C. Quenez, Backward stochastic differential equations in finance, Math. Finance, 7 (1997), pp. 1-71.

[22] M. Falcone And R. FerRetti, Semi-Lagrangian Approximation Schemes for Linear and HamiltonJacobi Equations, Other Titles Appl. Math. 133, SIAM, Philadelphia, 2013.

[23] H. Föllmer And P. Leukert, Quantile hedging, Finance Stoch., 3 (1999), pp. 251-273.

[24] F. N. Fritsch And R. E. CARlson, Monotone piecewise cubic interpolation, SIAM J. Numer. Anal., 17 (1980), pp. 238-246.

[25] E. Gobet And S. PAgliarani, Analytical approximations of BSDEs with nonsmooth driver, SIAM J. Financial Math., 6 (2015), pp. 919-958.

[26] V. Henderson, K. Kladínko, M. Monoyios, and C. Reisinger, Executive stock option exercise with full and partial information on a drift change point, SIAM J. Financial Math., to appear.

[27] K. IN'T Hout, Numerical Partial Differential Equations in Finance Explained: An Introduction to Computational Finance, Palgrave Macmillan, London, 2017.

[28] E. R. JaKobSEn AND K. H. KARLSEn, Continuous dependence estimates for viscosity solutions of integroPDEs, J. Differential Equations, 212 (2005), pp. 278-318.

[29] E. R. Jakobsen, A. Picarelli, and C. Reisinger, Improved order $1 / 4$ convergence for piecewise constant policy approximation of stochastic control problems, Electron. Commun. Probab., 24 (2019), pp. $1-10$.

[30] N. V. KRYLov, Approximating value functions for controlled degenerate diffusion processes by using piece-wise constant policies, Electron. J. Probab., 4 (1999), pp. 1-19.

[31] N. V. KRYLOV, On the rate of convergence of finite-difference approximations for Bellmans equations with variable coefficients, Probab. Theory Related Fields, 117 (2000), pp. 1-16.

Copyright (C) by SIAM. Unauthorized reproduction of this article is prohibited. 
[32] K. MA AND P. Forsyth, An unconditionally monotone numerical scheme for the two-factor uncertain volatility model, IMA J. Numer. Anal., 37 (2016), pp. 905-944.

[33] L. Moreau, Stochastic target problems with controlled loss in jump diffusion models, SIAM J. Control Optim., 49 (2011), pp. 2577-2607.

[34] A. Picarelli, C. Reisinger, and J. Rotaetxe, Boundary mesh refinement for semi-Lagrangian schemes, in Hamilton-Jacobi-Bellman Equations: Numerical Methods and Applications in Optimal Control, D. Kalise, K. Kunisch, and Z. Rao, eds., Radon Ser. Comput. Appl. Math. 21, De Gruyter, Berlin, 2018, pp. 167-188.

[35] A. Picarelli, C. Reisinger, and J. Rotaetxe, Some regularity and convergence results for parabolic Hamilton-Jacobi-Bellman equations in bounded domains, J. Differential Equations, 268 (2020), pp. 7843-7876.

[36] D. M. Pooley, P. A. Forsyth, And K. R. Vetzal, Numerical convergence properties of option pricing PDEs with uncertain volatility, IMA J. Numer. Anal., 23 (2003), pp. 241-267.

[37] C. ReIsinger, The non-locality of Markov chain approximations to two-dimensional diffusions, Math. Comput. Simulation, 143 (2018), pp. 176-185.

[38] C. Reisinger And P. A. Forsyth, Piecewise constant policy approximations to Hamilton-Jacobi-Bellman equations, Appl. Numer. Math., 103 (2016), pp. 27-47.

[39] C. Reisinger and J. Rotaetxe, Boundary treatment and multigrid preconditioning for semi-Lagrangian schemes applied to Hamilton-Jacobi-Bellman equations, J. Sci. Comput., 72 (2017), pp. 198-230.

[40] X. WARIN, Some non-monotone schemes for time dependent Hamilton-Jacobi-Bellman equations in stochastic control, J. Sci. Comput., 66 (2016), pp. 1122-1147.

Copyright $\odot$ by SIAM. Unauthorized reproduction of this article is prohibited. 